\title{
Evaluating the Performance of a Large-Scale Facial Image Dataset Using Agglomerated Match Score Statistics
}

\author{
Mounica Kamireddy
}

Follow this and additional works at: https://researchrepository.wvu.edu/etd

\section{Recommended Citation}

Kamireddy, Mounica, "Evaluating the Performance of a Large-Scale Facial Image Dataset Using Agglomerated Match Score Statistics" (2016). Graduate Theses, Dissertations, and Problem Reports. 5928.

https://researchrepository.wvu.edu/etd/5928

This Thesis is protected by copyright and/or related rights. It has been brought to you by the The Research Repository @WVU with permission from the rights-holder(s). You are free to use this Thesis in any way that is permitted by the copyright and related rights legislation that applies to your use. For other uses you must obtain permission from the rights-holder(s) directly, unless additional rights are indicated by a Creative Commons license in the record and/ or on the work itself. This Thesis has been accepted for inclusion in WVU Graduate Theses, Dissertations, and Problem Reports collection by an authorized administrator of The Research Repository @ WVU. For more information, please contact researchrepository@mail.wvu.edu. 


\title{
Evaluating the Performance of a Large-Scale Facial Image Dataset Using Agglomerated Match Score Statistics
}

\author{
Mounica Kamireddy
}

Thesis submitted to the College of Engineering and Mineral Resources at West Virginia University

In partial fulfillment of the requirements for the degree of Master of Science in Electrical Engineering

\author{
Jeremy Dawson, Ph.D., Chair \\ Bojan Cukic, Ph.D. \\ Thirimachos Bourlai, Ph.D.
}

Lane Department of Computer Science and Electrical Engineering

\author{
Morgantown, West Virginia \\ 2016
}

Keywords: Biometrics, Facial Recognition, Characteristics of a Dataset, Match

Score, ROC curve, Statistical Distance Measures

Copyright 2016 Mounica Kamireddy 


\title{
Abstract
}

\section{Evaluating the Performance of a Large-Scale Facial Image Dataset Using Agglomerated Match Score Statistics}

\begin{abstract}
Mounica Kamireddy
Biometrics systems are experiencing wide-spread usage in identification and access control applications. To estimate the performance of any biometric systems, their characteristics need to be analyzed to make concrete conclusions for real time usage. Performance testing of hardware or software components of either custom or state-of-the-art commercial biometric systems is typically carried out on large datasets. Several public and private datasets are used in current biometric research. West Virginia University has completed several large scale multimodal biometric data collection with an aim to create research datasets that can be used by disciplines concerning secured biometric applications. However, the demographic and image quality properties of these datasets can potentially lead to bias when they are used in performance testing of new systems. To overcome this, the characteristics of datasets used for performance testing must be well understood prior to usage.
\end{abstract}

This thesis will answer three main questions associated with this issue:

- For a single matcher, do the genuine and impostor match score distributions within specific demographics groups vary from those of the entire dataset?

- What are the possible ways to compare the subset of demographic match score distributions against those of the entire dataset?

- Based on these comparisons, what conclusions can be made about the characteristics of dataset?

In this work, 13,976 frontal face images from WVU's 2012 Biometric collection project funded by the FBI involving 1200 individuals were used as a 'test' dataset. The goal was to evaluate performance of this dataset by generating genuine and impostor match scores distributions using a commercial matching software Further, the dataset was categorized demographically, and match score distributions were generated for these subsets in order to explore whether or not this breakdown impacted match score distributions. The match score distributions of the overall dataset were compared against each demographic cohorts.

Using statistical measures, Area under Curve (AUC) and Equal Error Rate (EER) were observed by plotting Receiver Operating Characteristics (ROC) curves to measure the performance of each demographic group with respect to overall data and also within the cohorts of demographic group. Also, Kull-back Leibler Divergence and Jensen Shannon Divergence values were calculated for each demographic cohort (age, gender and ethnicity) within the overall data. These statistical approaches provide a numerical value representing the amount of variation between two match score distributions In addition, FAR and FRR was observed to estimate the error rates. These statistical measures effectively enabled the determination of the impact of different demographic breakdown on match score distributions, and thus, helped in understanding the characteristics of dataset and how they may impact its usage in performance testing biometrics. 


\section{Acknowledgement}

I would like to express my gratitude to my advisor and committee chair, Dr. Jeremy Dawson for giving me this valuable opportunity. It is my privilege to work under his guidance, insight and encouragement. My sincere thanks to Dr. Bojan Cukic and Dr. Thirimachos Bourlai for their suggestions and for being in my committee.

I take the opportunity to thank my dearest colleagues Ms. Nikhita Nadiminti and Ms. Loukhya Kakumanu for their immense support throughout this thesis. It is my pleasure working with you both.

I wish to extend my heartfelt thanks to my parents and my sister for their incredible love and encouragement. This would not have been possible without your constant moral support.

I would like to thank Mr. Jason Hooks and Mr. James Searls for helping me during initial days of work. I also wish to thank Ms. Neeru Narang for sharing her ideas where ever needed. Finally, I thank my cousin Mr. Vishruth Mullapudi for his grammatical suggestions. 


\section{Table of Contents}

Abstract

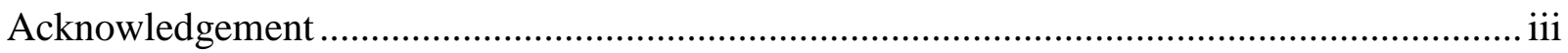

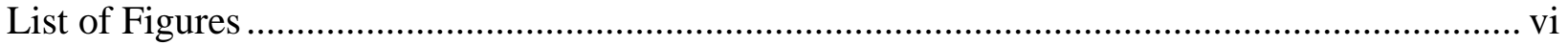

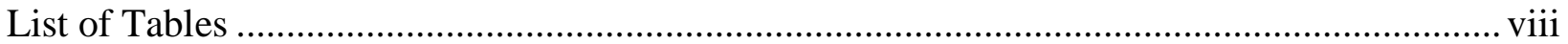

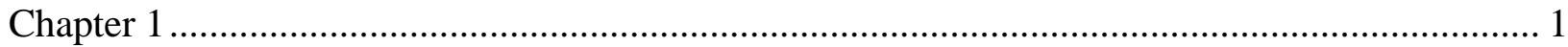

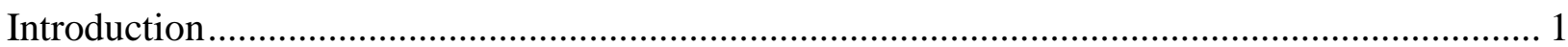

1.1 Face Recognition as a Biometric ...................................................................... 3

1.1.1 Advantages of Face Recognition....................................................................... 4

1.1.2. Characteristics of Face Recognition......................................................................... 5

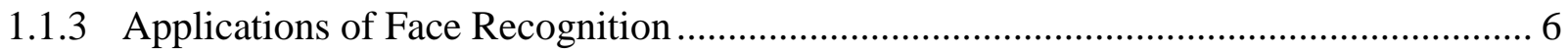

1.2 Challenges in Facial Recognition.............................................................................. 7

1.2.1 Approaches to Overcome Challenges .................................................................... 8

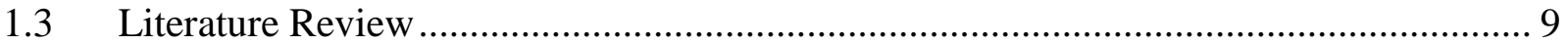

1.3.1 Comparative Study of Different Face Databases ................................................. 12

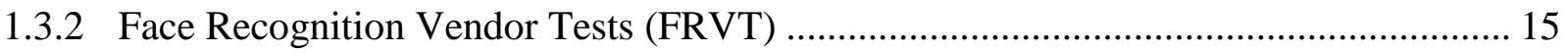

1.3.3 Face Recognition Grand Challenge (FRGC) ................................................... 16

1.3.4 Summary of Research Results of Various Databases .......................................... 18

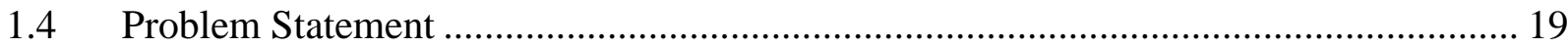

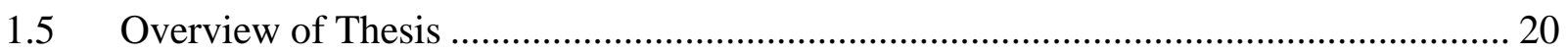

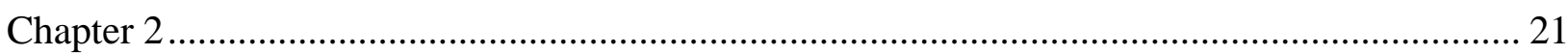

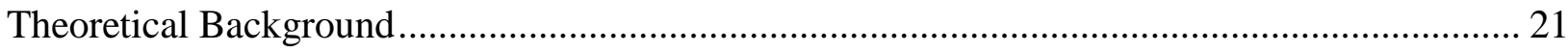

2.1 Illustration of Matchscore Distribution ............................................................... 21

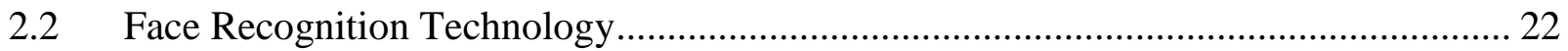

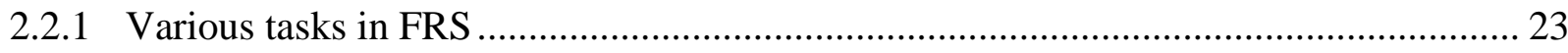

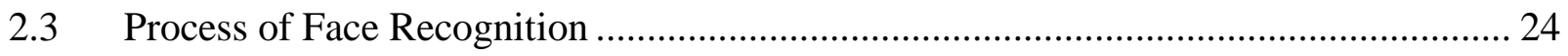

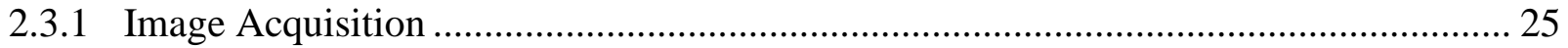

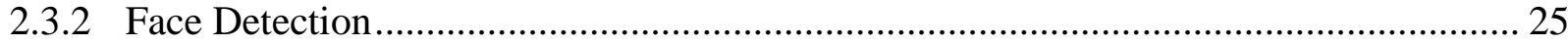

2.3.2.1 Viola-Jones Face Detector ........................................................................... 26

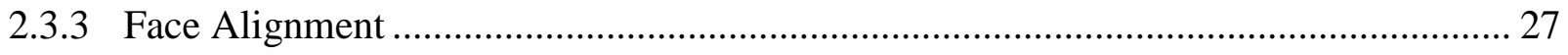

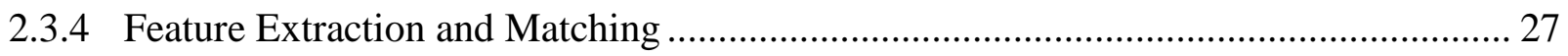




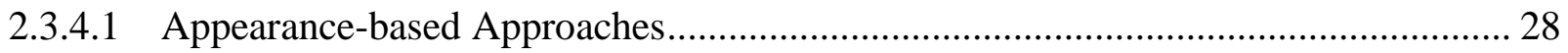

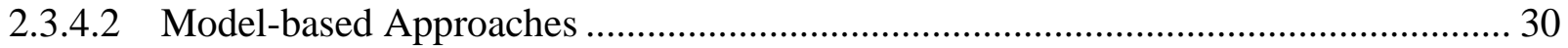

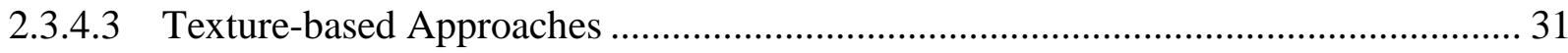

2.3.4.4 Comparison of various feature extraction techniques .......................................... 32

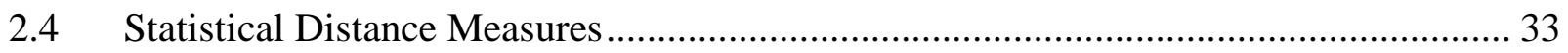

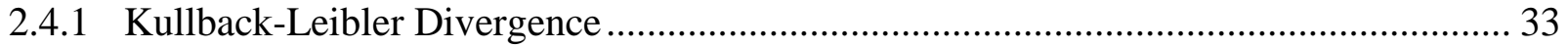

2.4.1.1 Properties of Kullback-Leibler Divergence..................................................... 34

2.4.2 Jensen-Shannon Divergence ......................................................................... 34

2.4.2.1 Properties of Jensen-Shannon Divergence ...................................................... 35

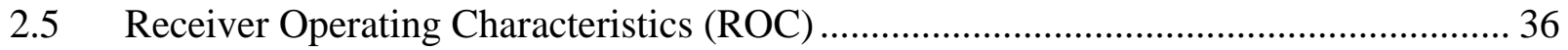

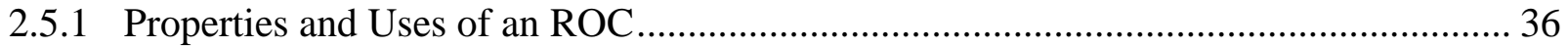

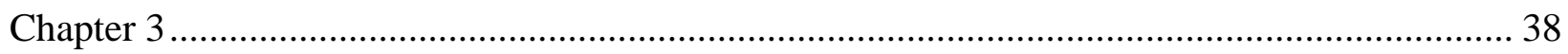

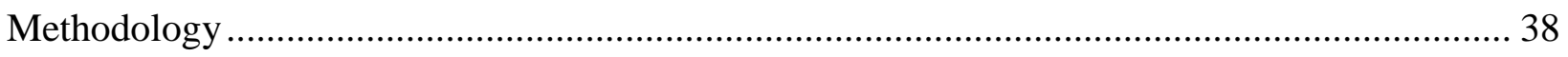

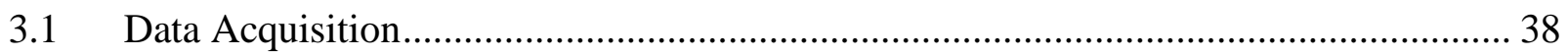

3.2 Commercial Matching Software ........................................................................ 40

3.2.1 Mega Matcher Matching Software ............................................................................ 41

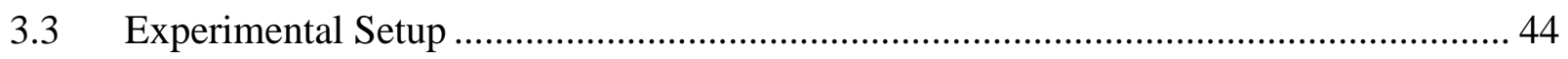

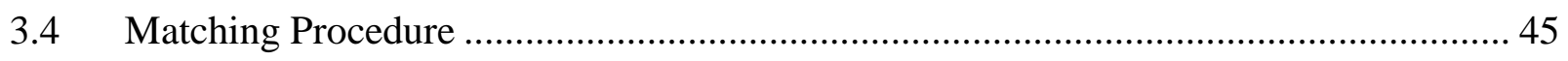

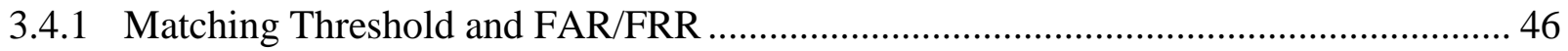

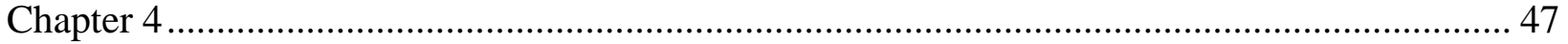

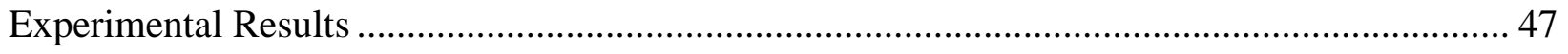

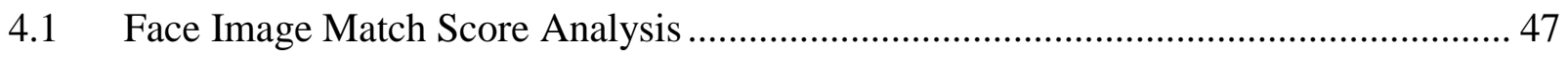

4.2 Demographic Break Down of Face Match Scores ................................................... 49

4.2.1 Gender-Based Face Match Score Distributions .................................................. 49

4.2.2 Age-Based Face Match Score Distributions ............................................................. 54

4.2.3 Ethinicity-Based Face Match Score Distributions ................................................. 60

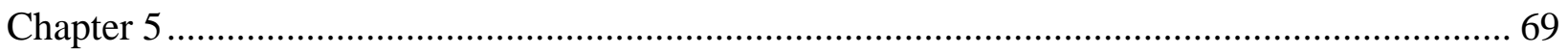

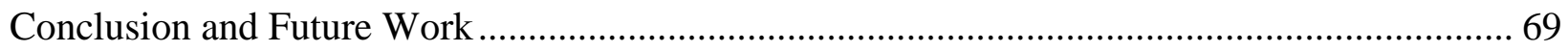

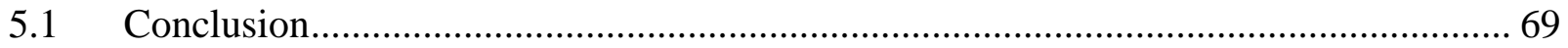

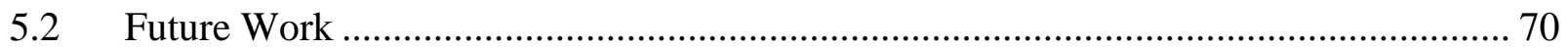

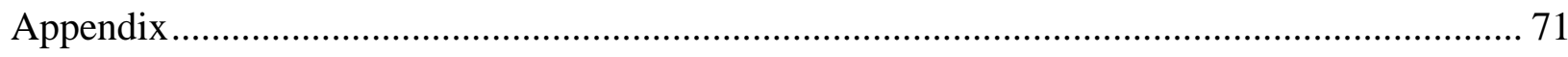

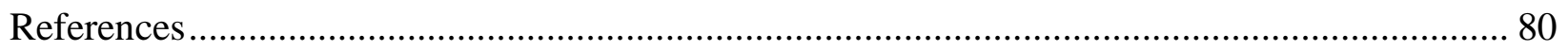




\section{List of Figures}

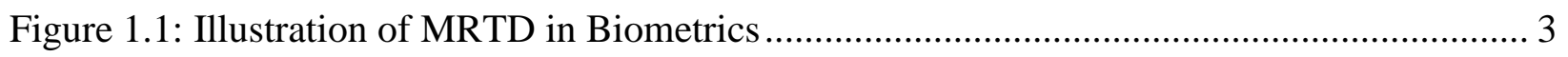

Figure 1.2: Intra-subject variation in a face ................................................................. 7

Figure 1.3: Reduced error rate for state-of-art recognition algorithms................................... 16

Figure 2.1: Gaussian distribution of the Genuine and Impostor User Populations .................... 21

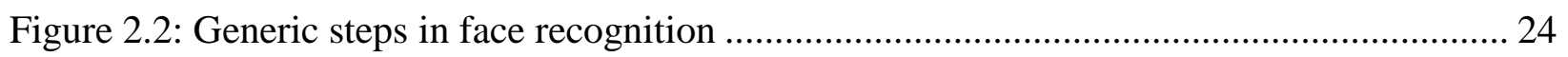

Figure 2.3: Representation of Viola-Jones face detection Haar like filters .............................. 26

Figure 2.4: Classification of face recognition techniques.................................................. 27

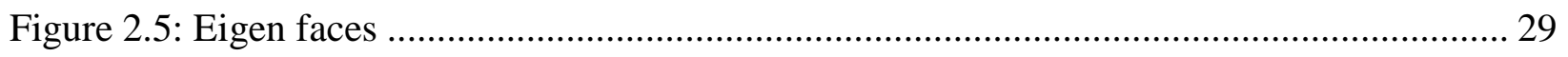

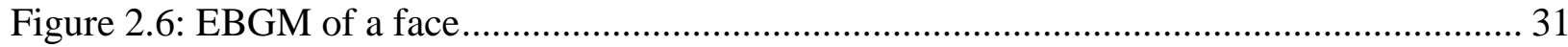

Figure 3.1: Schema of NeuroTechnology Biometric SDK ............................................... 41

Figure 3.2: Data Flow diagram of application based on VeriLook Standard SDK ................... 43

Figure 4.1: Match score distributions for face images from a Canon 5D Mark II digital camer. 48

Figure 4.2: Genuine and imposter score distributions for female face images......................... 50

Figure 4.3: Genuine and imposter score distributions for male face images ............................ 51

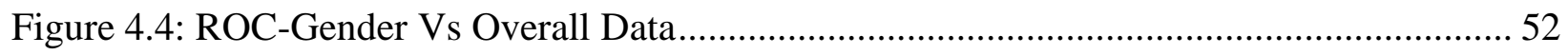

Figure 4.5: Graph of Distance score-Gender Demographics ............................................... 53

Figure 4.6: Genuine and imposter score distributions for Age 20-30 face images..................... 55

Figure 4.7: Genuine and imposter score distributions for Age 31-49 face images..................... 56

Figure 4.8: Genuine and imposter score distributions for Age 50-70 face images.................... 57

Figure 4.9: ROC Age Groups Vs Overall Data ................................................................. 58

Figure 4.10: Graph of Distance score-Age Demographics ................................................. 59

Figure 4.11-1: Combined match score distributions for ethnicities....................................... 62

Figure 4.11-2: Combined match score distributions for ethnicities...................................... 63

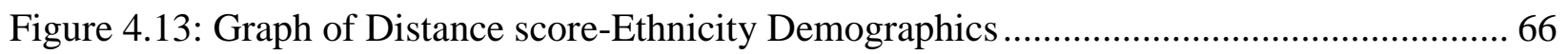

Figure A-1: Genuine and imposter score distributions for African face images ....................... 71

Figure A-2: Genuine and imposter score distributions for African-American face images ......... 72

Figure A-3: Genuine and imposter score distributions for Asian face images ......................... 73

Figure A-4: Genuine and imposter score distributions for Asian-Indian face images ............... 74 
Figure A-5: Genuine and imposter score distributions for Caucasian face images .................... 75

Figure A-6: Genuine and imposter score distributions for Hispanic face images ...................... 76

Figure A-7: Genuine and imposter score distributions for Middle-Eastern face images ............ 77

Figure A-8: Genuine and imposter score distributions for Pacific Islanders face images ........... 78

Figure A-9: Genuine and imposter score distributions for 'Others' face images ....................... 79 


\section{List of Tables}

Table 1.1: Summary of Various Face Databases .............................................................. 12

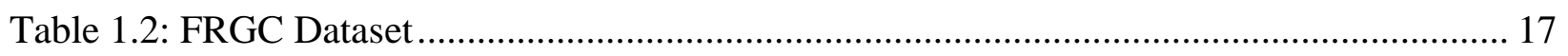

Table 1.3: Research Results on Various Databases ............................................................. 18

Table 2.1: Summary of Feature Extraction Techniques ..................................................... 32

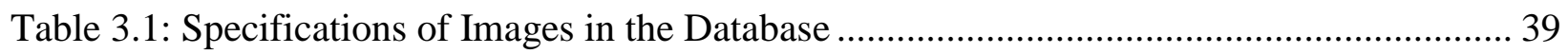

Table 3.2: Commercial Matching Software and their Tasks ................................................ 40

Table 3.3: Some of the Attributes in NeuroTechnology SDK .............................................. 44

Table 3.4: Matching Properties of Biometric Engine ........................................................ 45

Table 3.5: Relation between Matching Threshold and FAR ................................................ 46

Table 4.1: Summary of MatchScores for Overall Data ........................................................ 47

Table 4.2: Summary of MatchScores for Gender Demographics ........................................... 49

Table 4.3: Scores of attributes related to ROC-Gender Demographics ................................... 52

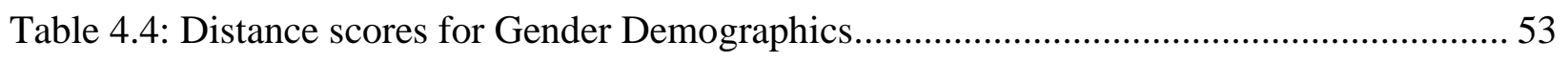

Table 4.5: Summary of MatchScores for Age Demographics ................................................ 54

Table 4.6: Scores of attributes related to ROC-Age Demographics ......................................... 58

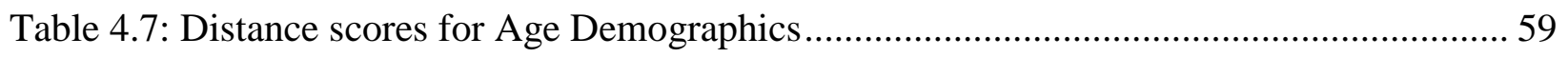

Table 4.8: Summary of MatchScores for Ethnicity Demographics ....................................... 61

Table 4.9: Scores of attributes related to ROC-Ethnicity Demographics .................................. 64

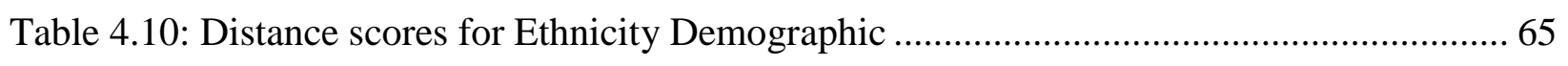

Table 4.11: Pairwise Comparison of Demographics MatchScores ......................................... 67

Table 4.12: Percentage of Error Rates Observed in the Dataset.............................................. 68 


\section{Chapter 1}

\section{Introduction}

In the recent past, the gap between mankind and physical human interactions which play a vital role in day to day life has widened. Striding much closer to an automated society, we find ourselves interacting more frequently with anonymous users and electronic information sources of the World Wide Web rather than with our human counterparts. Therefore identity has become a significant issue in the $21^{\text {st }}$ century. Even in wealthy, technologically savvy nations, identity fraud is an issue of increasing prevalence and threat. For such reasons, a rapid growth in the usage of biometric authentication in a wide range of market sectors has already begun. In the future, it will be desirable to have biometric scans become as common as swiping credit cards. Utilizing biometric authentication to provide the correct person with the proper access at the right time is essential for effective security.

Biometrics deals with the identity of an individual based on the physical, chemical or behavioral attributes such as fingerprints, face, hand geometry, iris, retina, signature, gait, palm print, odor, DNA etc. of the person [7]. In biometrics, all these attributes are termed as modalities. Over past few years, increasing security requirements have incentivized the development of several biometric options; the most common of which are fingerprint, iris and face. The following information gives a brief introduction to these prominent biometric modalities. Each has its own advantages and suits particular applications.

Fingerprint-based recognition has been the most successful and oldest method of biometric authentication. A fingerprint is composed of texture patterns of ridges and valleys. These ridges are characterized by numerous landmark points, termed as minutiae [17] and the spatial distribution of these minutiae points is unique for each finger. These minutiae points plays a vital role in matching two fingerprints along with sweat pores. Almost all forensic and law enforcement agencies are now employing Automated Fingerprint Identification Systems (AFIS). Fingerprint scanners are portable, light and relatively cheap, making them possible for incorporation into a wide range of mobile devices. All of these advantages have led to the extended usage of the 
fingerprint modality in several civil and commercial applications. Here are some of its real time applications [52]:

- In 1999, the FBI implemented the Integrated Automated Fingerprint Identification System (IAFIS) in the USA, which is one of the world's largest biometric operations. Likewise in 2004, US- Visitor and Immigrant Status Indicator Technology (US-VISIT) was launched with the objective of integrating with IAFIS to provide robust border security.

- In 2010, Saudi Arabia announced the requirement of fingerprints for all visitors as a part of identification for border security.

- In Canada, fingerprint based immigration and border crossing systems were introduced in 2011.

Iris identification has been considered as one of the most promising method in biometric authentication. The iris is a protected internal organ that surrounds the pupil and sclera. The iris is composed of several complex texture patterns with numerous elements such as stripes, pits and furrows making its appearance very distinctive, even among identical twins and extremely tough to spoof [17]. Iris-based identification is being employed in a large number of personal identification systems such as border crossing systems because of its speed and accuracy [7]. Although the occurrence of false match is very rare because of iris complexity, false rejection rates are relatively high compared to other biometric traits [7, 17]. Also, capturing the iris from a distance is something that is yet to be accomplished despite efforts to achieve this goal. Iris-based identification has gained tremendous importance, especially in Middle-Eastern countries. Some of applications [52] of iris-based identification are

- In 2003, the UAE launched the first iris based recognition system at Dubai international airport and further extended it to the remaining international airports in the country.

- In Germany, Iris was recorded on arrival at airports to monitor visitors along with AFIS as a part of the Schengen visa for biometric identification.

- In 2004, Congo introduced an iris based identification system for rehabilitation of the excombatants of war to civil life, which was proved to be highly successful.

- Canada practiced the use of iris based identification systems in 2005 at airports to accelerate the process of customs and immigration for pre-approved travelers. 
Face recognition is one of the key biometric trait which has been gaining more and more attention in research and industry during the past several years because of its wide variety of applications in both law enforcement and non-law enforcement activities. Among six biometric attributes considered by Hietmeyer[1], facial features secured high compatibility in the Machine Readable Travel Documents system based on factors such as enrollment, renewal, machine requirements and public perception (see Figure 1.1). In addition, when compared with some other identification technologies fair accuracy is seen in facial recognition. Also, the convenience of identification through non-contact process can be considered as one of the distinct advantages.

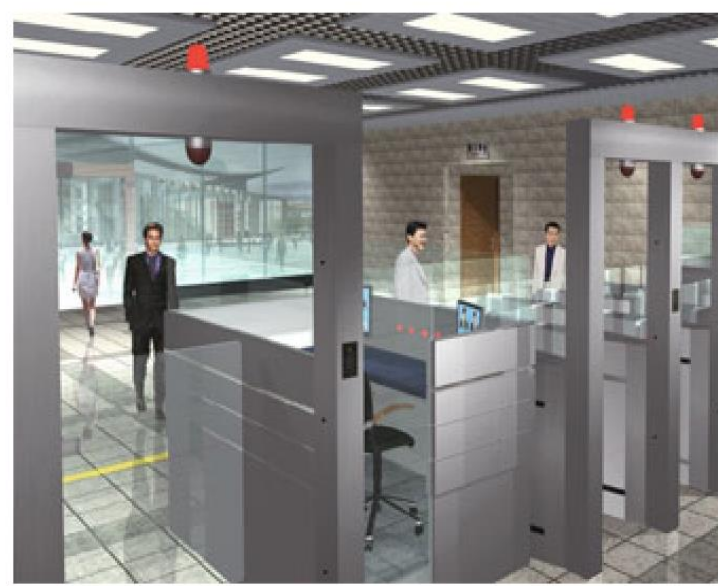

(a)

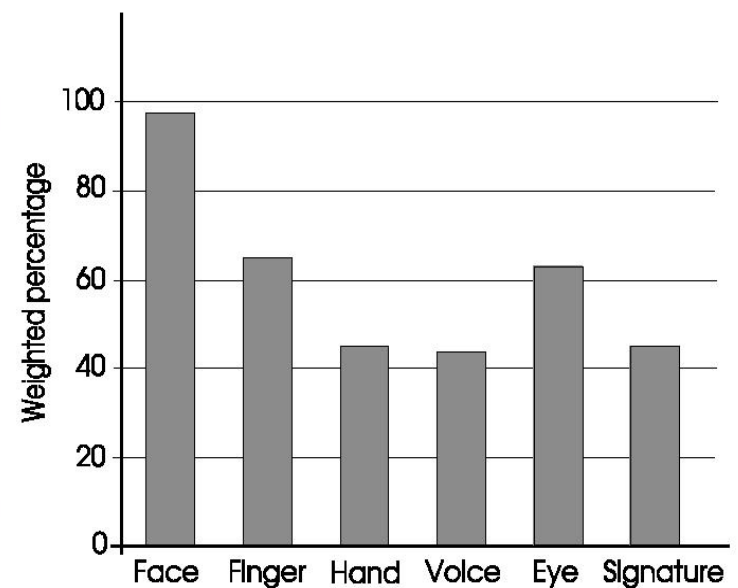

(b)

Figure 1.1: Illustration of MRTD in Biometrics.

(a) A scenario of using biometric MRTD for passport control (b) Comparison of various biometric traits based on MRTD compatibility [11]

\subsection{Face Recognition as a Biometric}

Every face has some distinguishable landmark points that enable us in differentiating people [11] such as: distance between the eyes, width of nose, length of jawline, shape of cheekbone, chin etc. The first automated version of a face recognition system was developed by Kanade in 1973 [11]. Since then efforts are being made to see significant changes in the performance of face recognition systems. However, there is need for further improvement. In the past, the term biometrics is strictly confined to address characteristics used in measuring features of biological organisms. Recently, 
variations in biological characteristics of a person which are used to differentiate people is termed as biometrics. Some such measured characteristics are now finding use in automated security and surveillance systems in order to verify individual's identity against some claimed persons at secured places or for searching pre-existing databases.

In addition to the above mentioned advantages, installation of biometric identification system in a place helps other advantages to emerge. For example, we hear cases such as a single person having managed to obtain multiple identities on a company payroll system. Such an issue can be handled easily with the help of biometric identification, which would notice duplicate biometric data. Without biometric system in a place, any intentional duplicity could be tough to prove even with extremely convincing evidence.

\subsubsection{Advantages of Face Recognition}

Although, face recognition does not necessarily suits all applications, it exhibits numerous key advantages over other biometrics such as [40]:

This modality is non-intrusive. Face recognition can be done by simply glancing into a camera from a distance. It is highly desirable to get the authentication done through a non-contact process to make the process convenient to the customers. By using strategic cameras placement, recognition can be performed even without a subject's knowledge.

Face recognition possesses numerous pre-existing databases. Amount of time required for collection of a biometric data and the accumulation of a database of sufficient size is one of the biggest constraints for the implementation of biometric identification. Large databases of high quality face images are already in place, unlike other biometric systems. Thus, the benefit of installing a face recognition system is gained readily after installation.

In general, face recognition systems have gained a higher level of public acceptance than most other biometric systems excluding in some nations. This is perhaps due to the non-intrusive nature of facial recognition, and also possibly the empathy undertones of technology recognizing the human face. Also, a subject needs to be more involved in other biometric systems. Such engagement is less required in face recognition. In order to integrate biometrics into government sectors, public acceptance is a key factor required for the systems to be implemented nationwide. 
Biometric data required for face recognition is easily interpretable by humans. A human reviewing a biometric source can manually check any identification or verification results. This contrasts with other biometric recognition systems, such as fingerprint or iris which needs an examination by an expert to draw a reliable conclusion. This can be viewed as a possible humanizing perception of face recognition.

\subsubsection{Characteristics of Face Recognition}

Biometrics characteristics are being used in a variety of applications. Face recognition has its own advantages and disadvantages. Characteristics of face recognition biometrics with respect to its pros and cons can be described as follows [7, 24]:

- Universality. Everyone has face, allowing almost every user to enroll apart from the rare exception of those with birth defects or other facial disfigurement.

- Uniqueness. Facial recognition is not very much unique. The probability of sharing common facial features between twins and among siblings or members of family is higher. Thus face-based recognition can be unreliable.

- Permanence. Face exhibits large intra-class variation (i.e., the same individual appearing different at different times). This is a drawback for face based recognition. These variations may be due to aging or voluntary desire to make changes. Constant reenrolling could be a possible solution for this issue.

- Collectability. There are several pre-existing large face databases which seems to be an ideal characteristic. However, certain measures are being taken while capturing an image such as maintaining sufficient resolution, proper angle and distance along with accessories which could be an obstacle. Also, some recognition systems demand frontal images which is impractical for real time applications.

- Performance. As seen earlier, face recognition biometrics are not unique, which results in inaccurate matches. False acceptance is higher in this trait compared to many other biometric traits. However, measure are being taken to improve matching accuracy. The overall performance may be deficient in benchmarks among face recognition systems currently in use.

Facial images often occupies large size due to the utilization of higher number of pixels and thus required extra storage space. Larger file sizes also requires higher processing time. 
A reduction in file size is not a solution to this issue because it results in poor quality image which is undesirable for accurate facial recognition.

- Acceptability. Overall, public acceptance is higher for face biometric trait. In general, facial images are often seen in passports and visas as a reference for identity which may not be common for other biometric traits. However, some social or religious customs may cause unwillingness to participate in facial recognition (e.g. women in the Middle East)

- Circumvention. Facial images can be easily spoofed. Also, concealing essential facial features needed for recognition is a major issue. This could be achieved by including liveness detectors.

\subsubsection{Applications of Face Recognition}

Internationally, biometric authentication systems have been employed in various parts of world since the last decade [52]. Some of them are listed below.

- In 2008, FBI expanded the existing database with Next Generation Identification (NGI) to include multi-modal biometrics (fingerprint, face, iris and palm patterns).

- The Mexican government announced the requirement of a new biometric identification card which carries fingerprints, a retina scan and a face to fight corruption in social programs in 2009.

- Venezuela launched a National ID card with facial and fingerprint data, streamlining the civil registry for the purpose of its electoral system and civil transactions in 2007.

- In between 2007-2008, Chile implemented the biometric research laboratory to validate facial/iris identification database in order to benchmark search algorithms for $1: \mathrm{N}$ in a 16 million record database.

- UK started issuing first generation e-passports with biometric data including fingerprints, iris and facial information to British citizens in 2006. Second generation e-passports with enhanced security features were introduced in 2010.

- In 2009, India launched the ambitious bio-enrollment project under the name of "Aadhar" with an aim to provide identity to its 1.2 billion population. The goal of this program is to issue identity with fingerprint, iris and facial information to all its citizens and ensure rightful use of social funds. 
- Brazil launched a new generation of smart cards called the Civil Identity Registry in 2011 with improved security features for fingerprint and facial biometric data, which are expected to replace current cards by 2019.

- In 2005, China adapted biometric technology for automated border crossing by installing biometric access gates between Shenzhen and Hong Kong which was later implemented near the Zhuau-Macau border in 2006.

\subsection{Challenges in Facial Recognition}

One of the greatest quality human beings possess is being able to memorize and recollect things. The remarkable ability of humans to recognize faces with large variations within the same subject led to the development of automated face recognition systems based on 2D images. However, the current state-of-the-art facial recognition technology functions only in a constrained environment. The main challenge in a vision based system is the presence of high degrees of variability in human facial images. There can be very large intra-subject variations due to head position, illumination, facial expression, aging [2,58] (see Figure 1.2), facial hair and small inter-subject variations due to similarity between individual appearances [40]. Imaging parameters such as exposure time, lens aberrations, aperture and sensor spectral response are also the factors that trigger intra-subject variations [11].
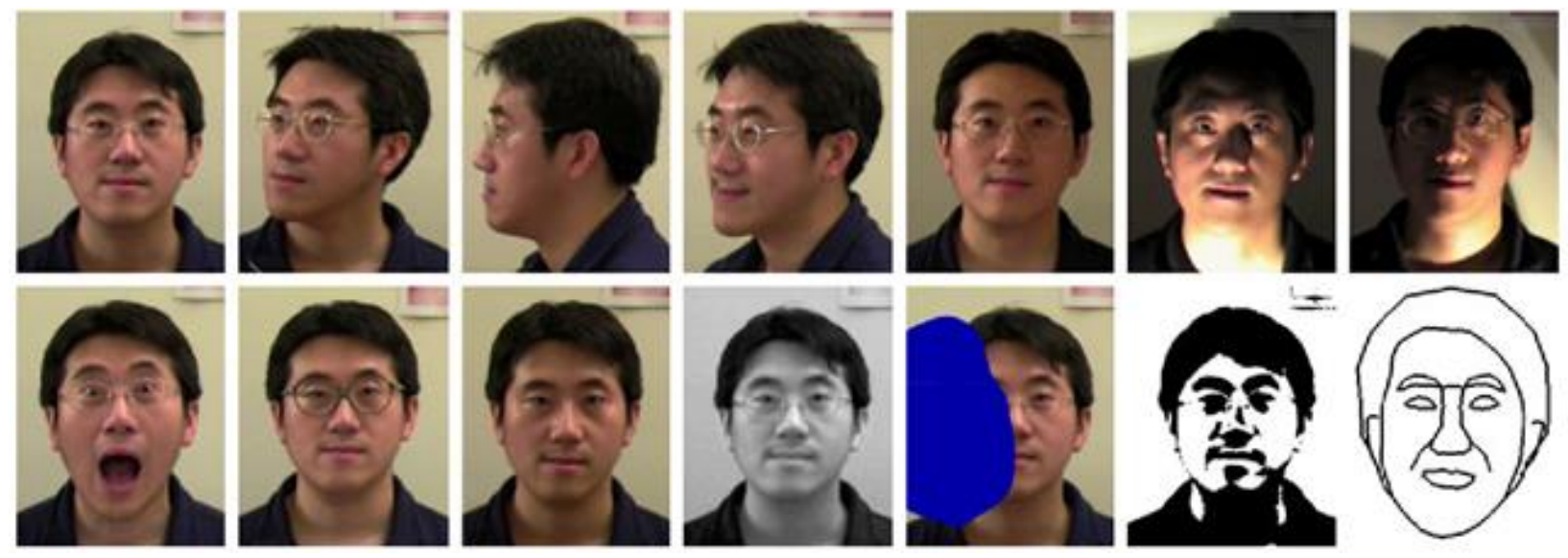

Figure 1.2: Intra-subject variation in pose, illumination, expression, occlusion, accessories color, brightness etc. [4] 
The face manifold of an individual is extremely non convex making it a complex issue challenging the accuracy of linear methods such as Principal Component Analysis, Linear Discriminant Analysis etc. Another challenge is related to high dimensionality and a small sample size. Dimensions of learning manifold usually differs with the size of image space and hence it cannot be generalized.

\subsubsection{Approaches to Overcome Challenges}

There are two possible solutions to handle the above mentioned challenges: feature extraction and pattern recognition [11]. Feature extraction is carried out in two steps: normalizing and extracting features from normalized images. Later strategy is through construction of a classification engine that helps in solving non-linearity issues. Ideal algorithm combines both these strategies.

All currently available vision based recognition techniques can be mainly classified as appearance based, which uses holistic texture features and geometry based which uses geometric features. Experimental results show that, better recognition task is seen in appearance based methods than in geometric-based recognition because of difficulty in extracting exact geometric features, especially in low quality images. Therefore, quality of image plays a major role in recognizing a face by a system. It is expected to maintain the quality sufficient to extract accurate facial features. With bad computation of facial features, the robustness of even the best algorithm deteriorates as the quality of image decreases. However, appearance based techniques exhibits poor performance with wide variations in head pose and illumination $[3,11]$. With changes in illumination, the same face can appear differently. Hence, uniform lighting conditions is desirable. The following are the approaches to overcome variations in illumination and pose respectively [4].

There are various methods to handle illumination problems. Some of them are heuristic approaches where an algorithm is constructed based on the symmetry of faces [4]. However, this algorithm only suits frontal faces. Statistical approach, which aims at analysis and extraction of underlying manifolds by applying statistical tools and through Multispectral imaging where, images are captured at specific wavelengths suitable to overcome illumination issues.

Pose variation problems are the other things to be dealt with. Compared to profile views or other angled images, frontal view images give more robust information. Problems appears when there is a need to recognize rotated images using frontal pose training data. Using larger data of training 
sets and building graphs which can link features to nodes and define the transformation needed to mimic face rotation could be possible solutions.

\subsection{Literature Review}

In this section we explore some of existing face recognition literature relevant to our work. Research in automated methods of face recognition is said to have begun in 1960s by Bledsoe [19]. Once a fully functional automated face recognition system was implemented by Kanade [11] in 1977, huge majority of face recognition research has focused on 2D images. Let us start with a brief overview of 2D face recognition methods including neural networks, Eigen faces, geometric analysis, and graph matching along with some of more recent approaches such as Line Edge Map and Support Vector Machine which are applicable mostly to frontal faces. The most relevant papers and databases for each category will be discussed briefly.

Eigenface also referred as Karhunen- Loève expansion, eigenpictures, eigenvectors and principal component is one of the most deeply explored approaches. Kirby and Sirovich [5] conducted an extensive research using this approach. According to them, pictures of faces can be represented efficiently using principal component analysis and any face image could be approximately reconstructed using a regular face image (eigenpicture) and a small collection of weights describing each face by taking the closest weight into consideration (Euclidean Distance). These weights are obtained by projecting the face image onto the eigenpicture. Later this approach was used by Turk and Pentland [6] for face detection and identification. Early work on Eigenfaces was extended to eigenfeatures [8] corresponding to facial features such as eyes, nose and mouth. It was stated that the system achieved 95\% recognition rate using the eigenfeature approach on a FERET database [31] comprising of 7562 images approximately from 3000 individuals. To summarize, we can say that Eigenfaces are more sensitive to appearance than eigenfeatures.

The Fisherface method of facial recognition was described by Belhumeru [9], which uses PCA and LDA to produce a linear projection into low dimensional subspace, similar to that used in eigenface method. However, Fisherface method takes the advantage of 'within-class' information to maximize class separation. This means that the training set of Fisherface method utilizes multiple images of each person to determine within-class variation, allowing any variation between images of the same person to be minimized in the classification process. This is the main advantage of the Fisherface approach over eigenfaces. Any small variations in lighting conditions, facial 
expressions etc. can be identified. Thus LDA is more resistant to the challenges facial recognition has been facing.

Neural Networks is attractive because of its ability to deal with non-linearity in networks. Gender classification, facial recognition and classification of facial expression can be resolved using Neural Networks. This approach for identification and verification of facial images was described by Lawrence et al [12]. A hybrid neural network involving local image sampling, a self-organizing map (SOM) and a convolution neural network was implemented by them. The SOM provides dimension reductions and invariance to minor changes in image sample. The convolution neural network extracts larger features in a batch of layers hierarchically and provides partial invariance to changes in rotation, deformation etc. This paper reported 96.2\%accurate recognition on an AT\&T database of 400images from 40 individuals.

There is a face recognition approach in which graphical representation of face image graph can be achieved. Information in graphs is encoded based on color, texture, edge maps etc. With graphs, an attempt is made to fold any variance between images of the same person, while emphasizing differences between different people. Recognition is then performed using graph matching techniques.

Wiskott et al [14] used an 'Elastic bunch graphs' method where an attempt was made to recognize faces from a large database holding single images of people, in spite of differences in facial expression and head orientation where variances are collapsed by extraction of face description in the form of graphs. Nodes of an image graph are taken from set of prominent points defined on face such as tip of nose, two corners of mouth, pupil etc. While other points defined as the center of gravity of the forehead, chin, cheek etc. Each of these nodes is labeled with a jet. A jet is a set of 40complex coefficients describing a small patch of grey values surrounding a single pixel, based on the Gabor wavelet transform. The issue related to head orientation is tackled by providing a different face bunch graph for all possible orientations. Thus, with the EBGM technique, head orientation issue was addressed by the authors. However, they didn't completely handle the problem of varying lighting conditions.

Here is yet another technique which is based on the set of geometric features computed from a facial image. This method follows a sequential order of facial feature localization and measurement of these features, characteristics and relative positions. According to Brunelli and 
Poggi [15], two faces could be distinguished based on geometrical features, such as the thickness and shape of eyebrow arches, width and vertical position of the mouth and nose, width of jaw and width of face across the cheek bone etc. The left and right boundaries of the face and nose are detected using horizontal gradients maps whereas vertical gradient maps are used to locate the top of the head, eyes, mouth and base of the nose. A template matching technique is used to locate the eyes. In the experiment conducted by Brunelli and Poggi [15], recognition rate of 90\% was recorded using a database of 47 individuals.

Several face recognition algorithms includes some basic template matching techniques. A template matching process uses samples, pixels or textures as patterns. It uses correlations or measures of distance. In the basic version of template matching, a test image represents a two dimensional array of intensity values. These intensity values are compared using Euclidean distance, with a single template representing a whole face. Also, one can use more than one face template from various viewpoints to represent a person's face and a face from a single view point can be represented as a set of multiple smaller templates. Brunelli and Poggi [15] compared template matching with geometric feature matching. They selected a set of four feature templates (eyes, nose, mouth and whole face) for all available faces in database containing 188 images of 47 individuals and observed a better recognition rate of $100 \%$ with template matching than in geometric feature matching's recognition rate of $90 \%$. However, the template matching technique is computationally complex. In general, template based techniques appears more logical compared to feature based techniques.

None of the existing techniques is free from limitations. Efforts are to be made to improve the performance of these techniques, keeping in mind larger databases and environmental conditions while conceiving a new technique or enhancing an existing technique. Here are some hybrid techniques being used currently.

Edge information is a feature representation object which is insensitive to illumination variations to some extent. Although this approach found use in several pattern recognition fields, it has been ignored in the case of face recognition. Gao et al [18] proposed a line edge map (LEM) technique which extracts lines from a face edge map as features. LEM can be stated as a hybrid of template matching and geometrical feature matching. This gains the advantages of invariance to 
illumination and a low memory requirement from feature matching approach and a high recognition performance and accuracy from template matching approach.

In a Support Vector Machine (SVM) [61], a training set of images is used to compute an Optimal Separating Hyper plane (OSH), thereby reducing the risk of misclassification between two classes of images in some feature space. To address the problem of face recognition, Guo et al [22] used SVMs with a binary tree recognition strategy in which a face image is iteratively categorized as belonging to one of the two classes, propagating until a binary tree structure denotes individual subjects on two classes and final classification decision can be made. This approach was tested on an ORL database of 400 images of 40 individuals, the average error rate recorded was $3 \%$ compared with $5.25 \%$ error generated by the normal eigenface method.

\subsubsection{Comparative Study of Different Face Databases}

As discussed earlier, one of the advantages face modality has is the existence of several publicly available databases containing face images captured under different conditions. Let us have a brief look at some of them and discuss in detail about each database and its limitations in Table 1.1 [19]

Table 1.1: Summary of Various Face Databases

\begin{tabular}{|c|c|c|}
\hline Database & Description & Limitations \\
\hline AT\&T [25] & $\begin{array}{l}\text { Contains } 400 \text { face images of } 40 \text { people } \\
\text { (10 each) and each captured at different } \\
\text { instances and different lighting } \\
\text { conditions, always against a dark } \\
\text { background }\end{array}$ & $\begin{array}{l}\text { Limited number of people; } \\
\text { uncontrolled illumination; lack } \\
\text { of images with different facial } \\
\text { expressions and head rotations }\end{array}$ \\
\hline $\begin{array}{l}\text { XM2VTS } \\
\text { [26] }\end{array}$ & $\begin{array}{l}\text { This database is designed to test face } \\
\text { and voice features of people. Video and } \\
\text { speech recordings of } 1000 \mathrm{~GB} \text { size } \\
\text { from } 295 \text { subjects over a period of } 4 \\
\text { months for } 4 \text { sessions (at an interval of } \\
\text { one month between sessions). } \\
\text { Significant variations such as change in } \\
\text { hairstyle, facial hair, accessories were } \\
\text { observed among the recordings. }\end{array}$ & $\begin{array}{l}\text { Doesn't disclose any } \\
\text { information related to image } \\
\text { acquisition parameters such as } \\
\text { pose, illumination angle etc. }\end{array}$ \\
\hline Yale [27] & $\begin{array}{l}\text { Contains } 165 \text { frontal grayscale images } \\
\text { collected from } 15 \text { people ( } 11 \text { each). } \\
\text { Captured under different lighting }\end{array}$ & $\begin{array}{l}\text { Limited number of subjects; } \\
\text { precise position of light was }\end{array}$ \\
\hline
\end{tabular}




\begin{tabular}{|c|c|c|}
\hline & $\begin{array}{l}\text { conditions (left, center, right); with and } \\
\text { without glasses and with variety of } \\
\text { facial expressions including normal, } \\
\text { happy, sad, sleepy, surprised and wink }\end{array}$ & $\begin{array}{l}\text { not mentioned; no variations in } \\
\text { pose angles were seen }\end{array}$ \\
\hline Yale B [28] & $\begin{array}{l}\text { Contains grayscale images of } 5760 \\
\text { images of } 10 \text { subjects ( } 64 \text { variety of } \\
\text { lighting angles }+9 \text { different poses). } \\
\text { Pose } 0 \text { is frontal view in which subject } \\
\text { concentrates camera lens i.e. } 0 \circ \text {; In } \\
\text { poses } 1 \text { to } 5 \text {, the subject gazes about } 12^{\circ} \\
\text { away from camera lens to his/her left } \\
\text { and poses } 6,7 \text { and } 8 \text { gazes } 24 \circ \text { away } \\
\text { from camera lens towards left. An } \\
\text { overhead lighting structure with } 64 \\
\text { computer controlled xenon strobe } \\
\text { lights were arranged. Capture rate was } \\
30 \text { frames/sec. }\end{array}$ & $\begin{array}{l}\text { Limited number of subjects; } \\
\text { the backgrounds are cluttered } \\
\text { and heterogeneous }\end{array}$ \\
\hline MIT [29] & $\begin{array}{l}\text { Covered } 16 \text { subjects. Captured for } 27 \\
\text { times when sat on a couch with various } \\
\text { head orientations. Lighting angles and } \\
\text { camera zoom were varied during the } \\
\text { sequence. Each raw image was a } \\
\text { grayscale image of } 480 \times 512 \text { which was } \\
\text { scaled to } 15 \times 16 \text { gray scale image using } \\
\text { X-by-Y pixel count in order overcome } \\
\text { size constraints }\end{array}$ & $\begin{array}{l}\text { Although variations in } \\
\text { lighting; pose and scale were } \\
\text { maintained; precise } \\
\text { measurements of each were not } \\
\text { mentioned. }\end{array}$ \\
\hline $\begin{array}{l}\text { CMU Pose, } \\
\text { Illumination, } \\
\text { Expression } \\
\text { (PIE) [30] }\end{array}$ & $\begin{array}{l}\text { Has images of } 16 \text { subjects that were } \\
\text { captured } 13 \text { different poses, } 43 \\
\text { different illumination conditions and } 4 \\
\text { different facial expression making a } \\
\text { total of } 41,368 \text { colored images with a } \\
\text { resolution of } 640 x 486 \text {. Two sets of } \\
\text { images one with ambient light and } \\
\text { other without ambient light were } \\
\text { captured. }\end{array}$ & $\begin{array}{l}\text { Cluttered backgrounds; exact } \\
\text { pose angle was not disclosed. }\end{array}$ \\
\hline $\begin{array}{l}\text { Bern } \\
\text { University } \\
\text { face } \\
\text { database } \\
\text { [20] }\end{array}$ & $\begin{array}{l}\text { Has frontal views of } 30 \text { people. } 10 \text { gray } \\
\text { images per person were captured with } \\
\text { various poses, two with frontal pose, } \\
\text { and two each looking on both (left and } \\
\text { right) sides and two each viewing up }\end{array}$ & $\begin{array}{l}\text { Limited number of subjects; } \\
\text { No images were captured } \\
\text { under varied illuminations }\end{array}$ \\
\hline
\end{tabular}




\begin{tabular}{|c|c|c|}
\hline & $\begin{array}{l}\text { and down. All were shot under } \\
\text { controlled conditions }\end{array}$ & \\
\hline $\begin{array}{c}\text { Purdue AR } \\
\text { [21] }\end{array}$ & $\begin{array}{l}\text { Contains } 4000 \text { colored frontal views of } \\
126 \text { people ( } 70 \text { men+56 women) } \\
\text { captured in two different sessions with } \\
\text { a span of } 14 \text { days between them. The } \\
\text { participants are free from restrictions } \\
\text { i.e. on clothing, accessories, make-up } \\
\text { or hair-style. Captured with various } \\
\text { facial expressions (neutral, smile, anger } \\
\text { and screaming), multiple illumination } \\
\text { conditions (left side on, right side on, } \\
\text { both sides on), partial facial occlusion } \\
\text { (sunglasses or a scarf) }\end{array}$ & $\begin{array}{l}\text { Position of light source is not } \\
\text { mentioned which may cause } \\
\text { glare in the image when } \\
\text { participant has glasses on. }\end{array}$ \\
\hline FERET [31] & $\begin{array}{l}\text { Created by FERET program with } 1000 \\
\text { subjects, between } 1993 \text { and } 1997 \text { in } \\
\text { order to support government monitored } \\
\text { testing and evaluation of face } \\
\text { recognition algorithms using standard } \\
\text { tests. The final version of database } \\
\text { consists of } 14051 \text { grayscale images } \\
\text { with frontal view, left and right profile } \\
\text { views and quarter left and right views } \\
\text { of head. Has images of same subject } \\
\text { with a gap of lyear in order to observe } \\
\text { changes in facial features. }\end{array}$ & $\begin{array}{l}\text { No information about } \\
\text { illumination conditions was } \\
\text { included; didn't provide wide } \\
\text { variety of pose variations. }\end{array}$ \\
\hline $\begin{array}{c}\text { Kuwait } \\
\text { University } \\
\text { face } \\
\text { database } \\
\text { (KUFDB) } \\
\text { [32] }\end{array}$ & $\begin{array}{l}\text { This is a collection of } 250 \text { gray scale } \\
\text { images captured from } 50 \text { people( } \\
\text { 5each).Images were normalized to } \\
\text { sizes } 24 \times 24,32 \times 32 \text { and } 64 \times 64 \text {. No } \\
\text { laboratory illumination conditions are } \\
\text { maintained. Variations in lighting, } \\
\text { facial expressions, size and rotation } \\
\text { were considered. }\end{array}$ & $\begin{array}{l}\text { Limited number of subjects; } \\
\text { didn't disclose any information } \\
\text { related to background and } \\
\text { pose. }\end{array}$ \\
\hline
\end{tabular}




\subsubsection{Face Recognition Vendor Tests (FRVT)}

\section{(I) $\quad$ FRVT 2002}

One of the large scale evaluations of automated face recognition is FRVT 2002 [33]. The main goal of this was to provide performance measurements in order to assess the ability of automatic face recognition systems to meet real-time recognition requirements. The soul of FRVT 2002 was a high computational intensity test which has 121,589 operational images of 37,437 individuals. Statistical performance was measured for all modes of verification, identification and watch list. The following conclusions were drawn from FRVT 2002.

- Indoor face recognition showed a viable improvement since FRVT 2000.

- Face recognition systems appeared insensitive to changes in indoor lighting.

- Males are easier to recognize than Females.

- Younger people seemed harder to recognize than older ones.

- Recognition rate from video sequence was less compared to the still imagery of FRVT 2002.

- Outdoor face recognition needs requires improvement.

- Observed improvement in ability to identify non-frontal faces

\section{(II) FRVT 2006}

FRVT 2006[34] is the sixth U.S Government sponsored evaluations of face recognition technology. This was an independent assessment performed by NIST. Some of the key features of this evaluation were

- Usage of high resolution 2D still images

- $\quad 3 \mathrm{D}$ images of both shape and texture channel were used

- Evaluated algorithm performance vs. human performance

- Simultaneously evaluated iris recognition

Three different datasets were used in FRVT 2006. They are

- First, a data set was collected during fall'04 and spring'05 at the University of Notre Dame consisting of high resolution frontal views captured under both controlled and uncontrolled illuminations. Participants arrives at weekly intervals throughout the academic year and two controlled and uncontrolled still images and one 3D image were captured. 
- Second, a multi biometric data set consisting of very high resolution still frontal images and $3 \mathrm{D}$ facial scans along with iris images.

- Third, a data set consisting of low-resolution JPEG images captured under uncontrolled illumination conditions. This was the same data set used in FRVT 2002.

Figure 1.3-2 shows the evaluation report in terms magnitude improvement in FRVT 2006 over FRVT 2002.

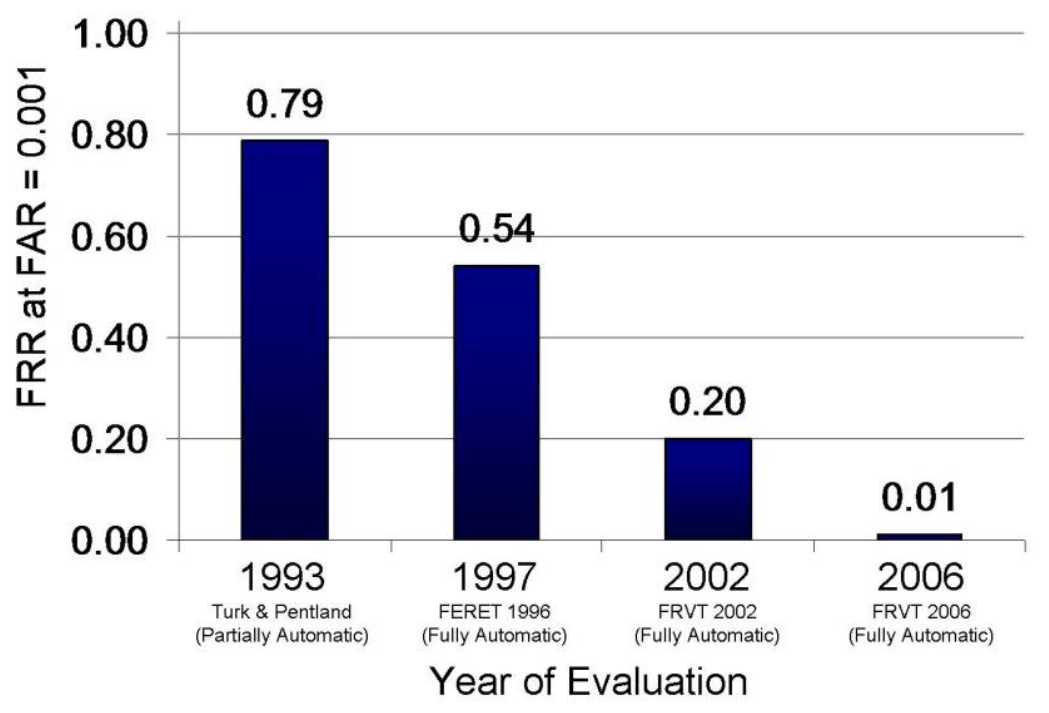

Figure 1.3: Reduced error rate for state-of-art recognition algorithms from 1993 through 2006 [34]

This indicates that best available algorithm in FRVT 2002 was $80 \%$ accurate whereas that in FRVT 2006 was $99 \%$ accurate both at a false acceptance rate of $0.1 \%$. This indicates a substantial improvement in technology. Also, the state-of-the-art algorithm in FRVT 2006 outperformed humans in identification tasks.

\subsubsection{Face Recognition Grand Challenge (FRGC)}

The FRGC [35] was intended to create a consistent research environment in order to show possible improvement over the FRVT 2002 results. The data for this experiment was collected at University of Notre Dame during academic years 2002-2003 and 2003-2004.

Table 1.2 explains the dataset used for the FRGC experiment 
Table 1.2: FRGC Dataset

\begin{tabular}{|c|c|c|c|}
\hline FRGC & \multicolumn{2}{|c|}{ Gallery Set } & Probe Set \\
\hline Data collected & \multicolumn{2}{|c|}{$\begin{array}{l}\text { Students at University of Notre } \\
\text { Dame (academic year 2002-2003) }\end{array}$} & $\begin{array}{l}\text { Students at University of Notre } \\
\text { Dame (academic year 2002-2003) }\end{array}$ \\
\hline Subsets & Gallery set 1 & Gallery set 2 & \\
\hline $\begin{array}{l}\text { Type of } \\
\text { images }\end{array}$ & $\begin{array}{l}\text { Still images } \\
\text { (2D) }\end{array}$ & $\begin{array}{l}\text { 3D images; } \\
\text { controlled 2D; } \\
\text { uncontrolled } \\
\text { 2D }\end{array}$ & $\begin{array}{l}\text { 3D images; controlled 2D; } \\
\text { uncontrolled 2D }\end{array}$ \\
\hline $\begin{array}{l}\text { Total number } \\
\text { of images }\end{array}$ & 12776 & 6601 & 32056 \\
\hline 2D controlled & 6338 & 3722 & 16028 \\
\hline $\begin{array}{c}2 \mathrm{D} \\
\text { Uncontrolled }\end{array}$ & 6338 & 1866 & 8014 \\
\hline $3 \mathrm{D}$ & 0 & 943 & 4007 \\
\hline $\begin{array}{l}\text { Number of } \\
\text { Subjects }\end{array}$ & 222 & 222 & 466 \\
\hline $\begin{array}{l}\text { Subject } \\
\text { sessions }\end{array}$ & $\begin{array}{l}\text { 9-16 per } \\
\text { subject }\end{array}$ & 943 & $\begin{array}{c}4007 \\
1-22 \text { per subject }\end{array}$ \\
\hline
\end{tabular}

Experiments were conducted on this data set to focus on number of key areas such as high resolution still 2D images, high resolution multiple still 2D image, 3D still images.

The following significant conclusions can be made based on results of FRGC

- Improvement in the performance of face recognition technology has been observed.

- The issue of uncontrolled environments needs to be addressed more carefully

- Performance of an average algorithm in FRGC is still lower than top performer of FRVT 2002

- 3D recognition using shape and texture need not necessarily provide better results than high quality $2 \mathrm{D}$ images. 


\subsubsection{Summary of Research Results of Various Databases}

In Table 1.3-3, a summary of performance evaluations of facial recognition algorithms on some of the existing databases is tabulated.

Table 1.3: Research Results on Various Databases

\begin{tabular}{|c|c|c|c|}
\hline Database & References & Method & $\begin{array}{l}\text { Percentage of Accurate } \\
\text { Recognition }\end{array}$ \\
\hline \multirow{2}{*}{ CMU PIE } & \multirow{2}{*}{ [16] } & PCA & $36.6 \%$ \\
\hline & & LDA & $95 \%$ \\
\hline \multirow{2}{*}{$\begin{array}{c}\text { CMU } \\
\text { Multi-PIE }\end{array}$} & \multirow{2}{*}{ [16] } & PCA & $63.3 \%$ \\
\hline & & LDA & $31.1 \%$ \\
\hline \multirow{2}{*}{ FERET } & \multirow{2}{*}{ [19] } & PCA & $\begin{array}{c}87 \% \text { in verification mode and } 54 \% \\
\text { in identification mode }\end{array}$ \\
\hline & & SVM & $\begin{array}{l}93 \% \text { in verification mode and } \\
77.78 \% \text { in identification mode }\end{array}$ \\
\hline \multirow{3}{*}{ AR } & [18] & LEM & $\begin{array}{l}96.43 \% \text { over a database containing } \\
\text { frontal views captured under } \\
\text { controlled conditions }\end{array}$ \\
\hline & \multirow{2}{*}{ [37] } & $\mathrm{SVM}+\mathrm{PCA}$ & $92.67 \%$ \\
\hline & & SVM+ICA & $94 \%$ \\
\hline \multirow[b]{3}{*}{ Yale } & \multirow{2}{*}[37,19]{} & $\mathrm{SVM}+\mathrm{PCA}$ & \multirow{2}{*}{$\begin{array}{l}99.39 \% \text { recognition rate with both } \\
\text { the methods over a database of } \\
165 \text { images of } 15 \text { people }\end{array}$} \\
\hline & & SVM+ICA & \\
\hline & [19] & $\begin{array}{l}\text { Combined holistic } \\
\text { and feature } \\
\text { analysis-based } \\
\text { approaches }\end{array}$ & $96.11 \%$ \\
\hline $\begin{array}{c}\text { Bern } \\
\text { University } \\
\text { Database }\end{array}$ & [18] & LEM & $100 \%$ \\
\hline \multirow[b]{2}{*}{ AT\&T } & {$[23,19]$} & $\begin{array}{l}\text { SVM with binary } \\
\text { tree }\end{array}$ & $\begin{array}{c}91.21 \% \text { for SVM and } 84.86 \% \text { for } \\
\text { nearest center classification }\end{array}$ \\
\hline & [13] & PDBNN & $\begin{array}{c}96 \% \text { using a database of } 200 \\
\text { individuals }\end{array}$ \\
\hline
\end{tabular}




\subsection{Problem Statement}

As discussed earlier, facial recognition being one of the prominent area in the fields of computer vision and pattern recognition, predicting the performance of face recognition systems is highly desirable. Facial recognition systems behave differently on various unseen data inputs. In order to test the validity of any matching algorithm, utmost care should be taken during data collection. Assessing the performance of matching algorithms on small sized databases can potentially lead to biased results. Hence, large scale databases are often required to ensure the performance of matching algorithms.

It is well documented that the characteristics of dataset used to develop and test algorithms, systems can impact the performance. Several factors pertaining to this issue are: pose, illumination, expression, background, etc. Other factors such as image quality (e.g. resolution, compression, blur), aging [58] and occlusions also contribute to facial recognition errors. Earlier studies have discovered the fact that specific demographic groups (e.g. gender, age, ethnicity), certain cohorts are more susceptible to errors [59]. In order to overcome these issues, characteristics of a dataset should be well understood prior to its real-time usage. To address this issue, methods that can provide indicators of the characteristics of a biometric dataset that may impact its performance if used in evaluation of a biometric system were developed.

The goal of this thesis is to determine the characteristics of the frontal face image data from the WVU 2012 BioCOP Collection that may impact results if this dataset is used to test a new biometric algorithm or system application.

The proposed goal was accomplished by completing the following tasks:

- Generating genuine and impostor match score distributions to see if they reflect the baseline performance of an ideal dataset.

- Comparing demographic match score distributions with those of the entire dataset to understand the effect of demographic breakdown.

- Compare the generated demographic match score distributions against those of the entire dataset using statistical measures to

- Based on these comparisons, draw conclusions about how the characteristics of this largescale dataset may impact its effectiveness in testing biometric systems. 


\subsection{Overview of Thesis}

This section gives a brief overview of the face as a biometric modality, its advantages, disadvantages and applications. Also, the evolution of existing algorithms and their implementation on various databases were discussed. The rest of work is organized as follows:

- Chapter 2: Brief summary of the basic terminology related to biometrics, various tasks in face recognition technology and detailed explanation on working of face recognition systems involving face detection and feature extraction. Basics on importance of Receiver Operating Characteristics and Statistical Distance Measures were discussed.

- Chapter 3: Detailed explanation of NeuroTechnology MegaMatcher SDK, its attributes, and properties along with experimental setup and matching procedure along with discussion on generation of genuine and impostor distributions. Also, data acquisition and its usage in thesis was described.

- Chapter 4: Summary of the experimental results of acquired biometric data on the complete dataset and then with demographic breakdown.

- Chapter 5: This chapter summarizes the contribution of this thesis. We draw conclusions from obtained results and discuss the most promising extensions to this work in the future scope section that follows. 


\section{Chapter 2}

\section{Theoretical Background}

\subsection{Illustration of Matchscore Distribution}

The general representation of biometric match scores are shown in figure 2.1. The basic terminology [35] associated with this illustration follows

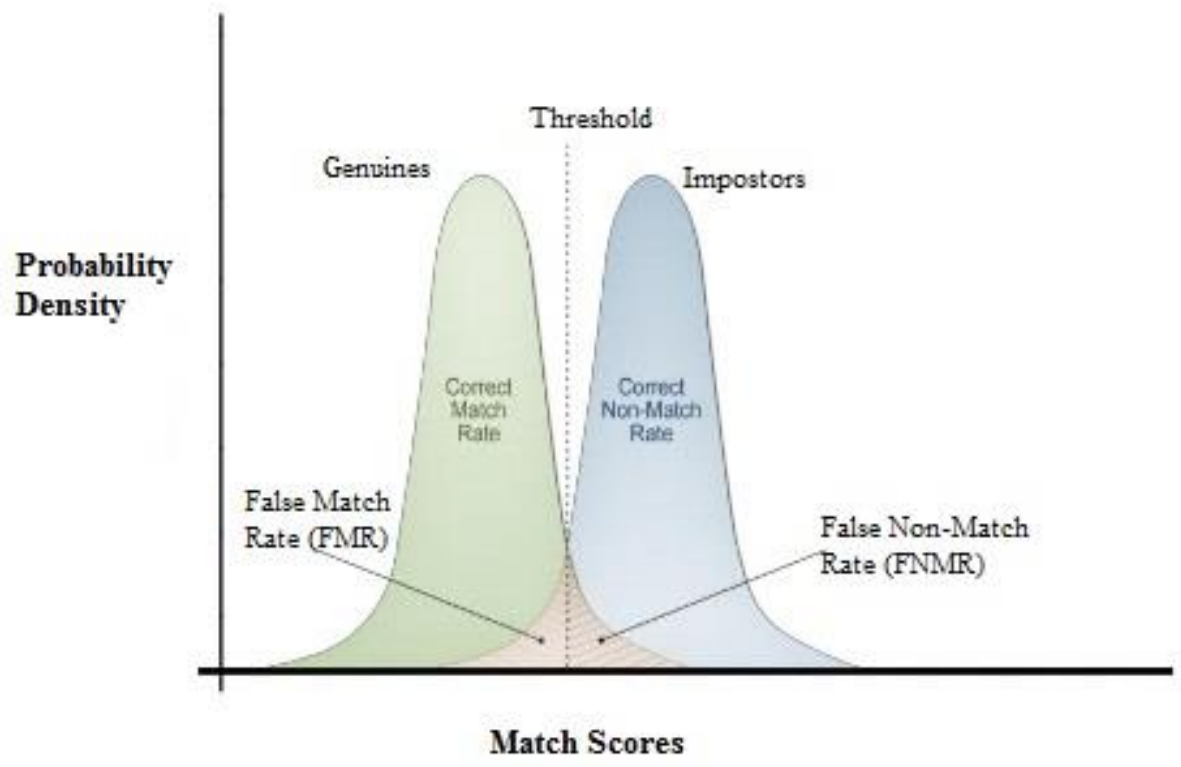

Figure 2.1: Gaussian distribution of the Genuine and Impostor User Populations [52]

- Biometric probes are the biometric characteristics extracted during verification or identification which are passed through an algorithm that convert the characteristic into biometric features to perform matching with biometric template.

- Biometric templates are sets of stored biometric features used to match against a biometric probe.

- A Genuine Score is defined as a match score recorded when the biometric probe of an individual matches with the biometric templates of the same individual in a database.

- An Impostor Score is defined as a match score recorded when the biometric probe of an individual matches with the biometric templates of the every other individual in a database. 
- A Match Score can be defined as a numeric value resulting from the comparison of a biometric probe with a biometric template.

- The Threshold is a numeric value at which decision boundary exists.

- The False Accept Rate (FAR) can be defined as the percentage of times a face recognition algorithm falsely accepts an incorrect claim as to the existence or non-existence of an individual in a database. This is a statistic used to measure the biometric performance of a task.

- The False Reject Rate (FRR) can be defined as the percentage of times a face recognition algorithm falsely rejects a correct claim of existence or non-existence of an individual in a database. This is a statistic used to measure the biometric performance of a task.

- Normalization is the process of adjusting the size, scale, illumination and orientation of both the biometric probe and biometric template to ensure commensurability.

- A Similarity Score is a value which indicates the similarity or correlation between a biometric probe and stored biometric templates in a database.

\subsection{Face Recognition Technology}

Face Recognition Technology (FRT) is a subfield of Pattern recognition technology, which uses statistical techniques to perform detection and extraction of patterns from data in order to match with stored patterns in database. The data upon which the pattern recognition system is performing should be in a way not to allow human to identify. It is important for a system to be able to locate and analyze an image pattern of a face which is free from noise.

A face recognition process follows a series of steps. The first step is the capturing of a facial image, technically termed the "probe image" which would normally be done with the help of a video or still camera with or without the knowledge of the subject. This is certainly one of the greatest features of FRT. The quality of the captured face image determines the efficiency of the whole system. The process begins with face detection and extraction from an image which normally contains the background, other faces and even more complex patterns. Such an image is normalized to the extent possible while retaining its actual properties (rotation, size etc.) The normalized face image is then passed to a recognition software where features are extracted to 
create a biometric template or represented mathematically in order to compare against those in the gallery.

\subsubsection{Various tasks in FRS}

Face Recognition Systems (FRS) can normally be used for three different tasks or combinations of tasks: Verification, Identification and Watch-list [35]. Each of these characterizes distinctive challenges while implementing FRT.

\section{(I) Verification (“Am I the identity I claim to be?"):}

This is also called authentication which is the simplest task for a FRS. This is meant for applications requiring user interaction in the form of an identity claim which is a one-to-one matching task as the system need not necessarily check every record in the database. The verification task is conducted for both

- Clients, people who try to gain access using their own identity.

- Impostors, people who try to gain access using a false identity.

The percentage of clients rejected access is reported as the FRR, and the percentage of impostors gaining access is reported as the FAR for a given threshold.

\section{(II) Identification ("Who am I or What is my identity?")}

Identification is a more complex task than verification. This is mostly meant for surveillance applications where user interaction is not essential or desired. Identification is a one-to-many problem which can be further classified as

- Closed-Set, an identification problem where we need to identify a known person in the gallery.

- Open-Set, an identification problem in which the identity of a person to be identified may or may not exist in the gallery.

Most real-time identification tasks tends to be open-set problems. The percentage of correct identification is reported as the true identification rate (TIR) and the percentage of wrong identification is reported as the false identification rate (FIR). 


\section{(III) Watch-list ("Is this one of the suspects we are looking for?")}

A watch-list task is a specific case of the open-set identification task. In this task, the system determines whether the probe image corresponds to a person on the watch list, and then subsequently identifies the person through the match. This is a one-to-many problem with an openset assumption.

In a watch-list task, along with TIR and FIR, the sensitivity of the watch-list (i.e. how often an unknown is classified as a person belonging to watch-list) can be described with the FAR. The converse holds well with the FRR.

The matching of a probe against a template in the gallery produces a similarity score. Based on this an identity claim is accepted when the similarity score meets the threshold and rejected if it fails to meet the threshold criteria. If the threshold for a similarity score is set too high, the FRR increases; similarly, if it is too low the FAR increases. In order to avoid these situations, a tradeoff must be considered while setting the threshold.

\subsection{Process of Face Recognition}

Face Recognition involves several concerns regarding its classification. Ultimately, the following classification was agreed upon by most of researchers. Face recognition is performed using the following steps $[3,24,11]$.

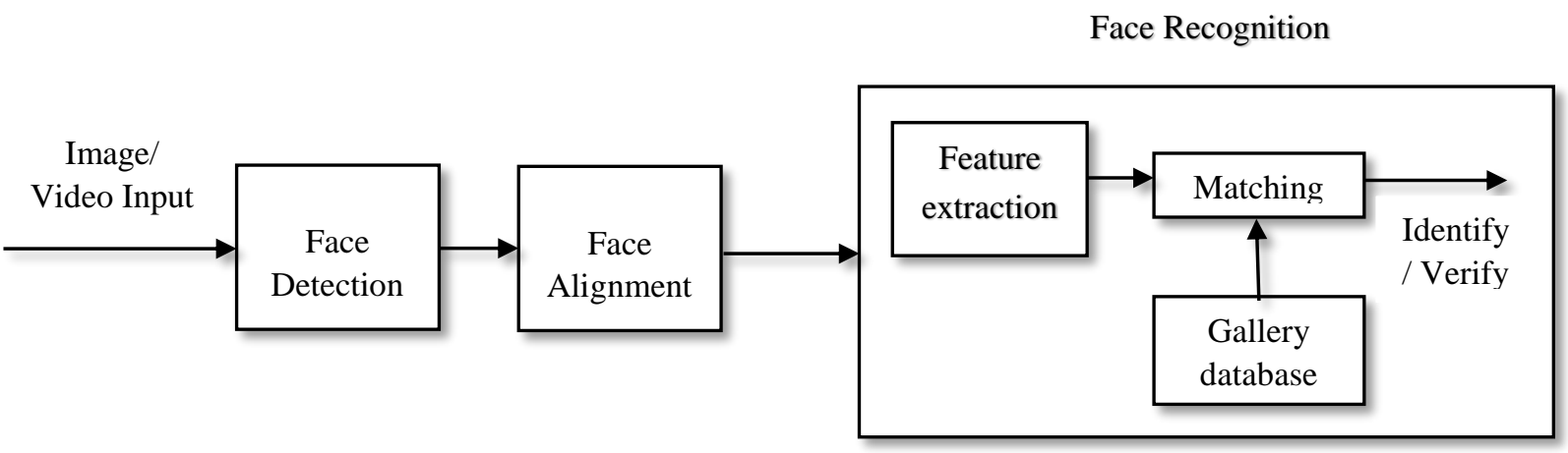

Figure 2.2: Generic steps in face recognition

The input of a face recognition system is either a still image or a video stream. This fed input is detected, and the process of detecting face from an image is followed by face alignment, feature extraction and matching. Feature extraction involves the extraction of relevant facial features from a normalized data. Let us discuss each step in detail. 


\subsubsection{Image Acquisition}

Face data used by automated face recognition systems should be in machine readable format. In general, the image formats used by these systems are 2D photos, 3D depth images and video sequences. In order to meet the growing demand and to address existing challenges, sensing techniques are continuously being improvised by capturing facial images in multiple spectra such as visible, infrared, near infrared etc.

- Image Inputs. Until recent advancements in face recognition systems, two dimensional visible spectrum images were the only source used by conventional automated systems. Therefore, most of the sensors and algorithms have been developed based on these requirements. 2D sensors are utilized in most applications. To overcome the challenges posed by 2D photographs, prevailing technology is being substituted with 3D sensors. An image captured by a 3D sensor typically covers $120^{\circ}$ of the human head, which is referred as a $2.5 \mathrm{D}$ scan [24]. Three to five such $2.5 \mathrm{D}$ scans completes a full $3 \mathrm{D}$ face model. However, usage of this technology is yet to become widespread.

- Video sequences. Face recognition can use video stream inputs along with still images. In case of video input, the system should be capable of not only detecting but tracking faces which is essentially a motion estimation problem. Face tracking can be performed using different methods, e.g., head tracking, feature tracking, model-based tracking, and imagebased tracking [3, 24]. The elementary face tracking process seeks the location of an image in a given picture. Then, variation between the frames is calculated to locate a face. This process may face some issues with partial occlusion, changes in illumination, computational speed, etc.

\subsubsection{Face Detection}

The main aim of face detection is to localize a face in an image. This is the foremost step in many face related applications. In general, processed images from a database are fed as input to a face detection algorithm. If the input is a video stream, facial tracking is done between frames, in order to preserve the identity of a subject and to reduce computational complexity. There is a lot of discussion related to classification criteria. Present state-of-art face detection methods are primarily based on local texture features from a given image followed by application of classifiers to distinguish between face and non-face objects [24]. The face detection algorithm proposed by 
Viola and Jones has been found successful in most real time applications. However, there is an ambiguity related to its robustness due to the production of false positive and negative errors.

\subsubsection{Viola-Jones Face Detector}

The Viola-Jones face detector scans through input images using windows of different sizes and checks whether or not a face exists in each window. The detection process can be accomplished with the help of scanning windows of various sizes. The existence of face in a window is determined by applying classifiers to local features derived using rectangular filters. These rectangular filters can be grouped as two-rectangle, three-rectangle, and four-rectangle [24] as shown in figure 2.3 .

These 2D rectangular filters are similar to the one dimensional Haar wavelets used in signal processing domain. Hence, these filters are also known as Haar-like filters [24, 39]. The difference between sum of pixel intensities in shaded and non-shaded regions gives the feature values. When the combination of filter features in certain window exceeds a threshold, a face is said to have been detected.
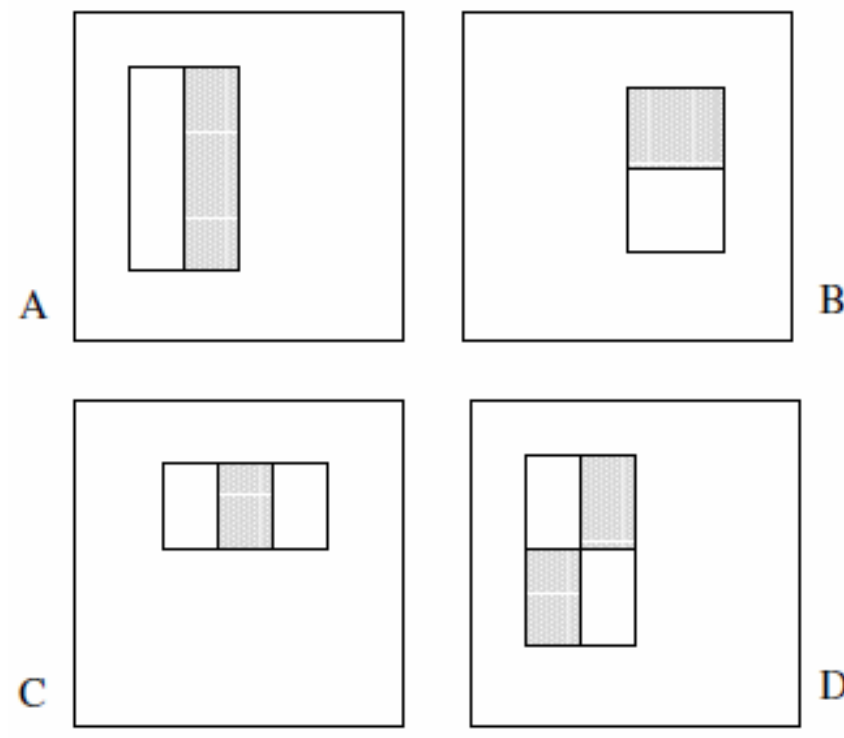

B

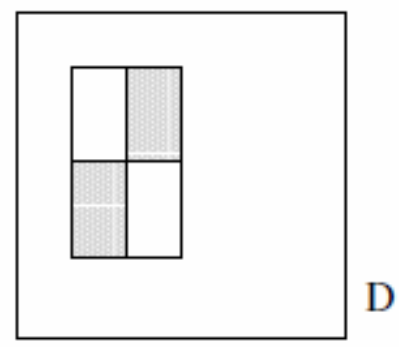

\section{Figure 2.3: Haar-like filters for face detection in Viola-Jones face detection (A) and (B) Two- rectangular filters (C) Three-rectangular filter and (D) Four-rectangular filter [38]}

However, a single Haar-like filter cannot accomplish the face detection task with high accuracy. Hence, a set of various sized Haar-like filters needs to be applied to each window and the combined 
filter response should be considered in detecting a face [24, 39]. Also, this approach can be used to detect individual components such as eyes within a detected face.

\subsubsection{Face Alignment}

Face alignment is a processing step prior to face recognition. This is performed in order to achieve more accurate localization and normalization of a face [11]. Based on location points, facial components such as eyes, mouth, nose and facial outline are located. Input image is normalized with respect to geometrical properties such as size, pose using geometrical transforms. Such an image is further normalized with respect to photometrical properties such as illumination and gray scale [11].

\subsubsection{Feature Extraction and Matching}

A feature extraction algorithm deals with extraction of features from data. It creates new features through combinations or transformations of original data. There are numerous feature extraction algorithms. Most of them find their use in other areas besides face recognition. The current available face recognition algorithms are updated versions of the ones developed years ago. One of these algorithms is PCA which was first invented a century ago, but proposed for pattern recognition in 1965 [50]. Finally, it was applied to facial recognition in the early 90's [5, 6].

There are three main approaches to extract features and match face images. Figure 2.4 illustrates the classification of feature extraction techniques.

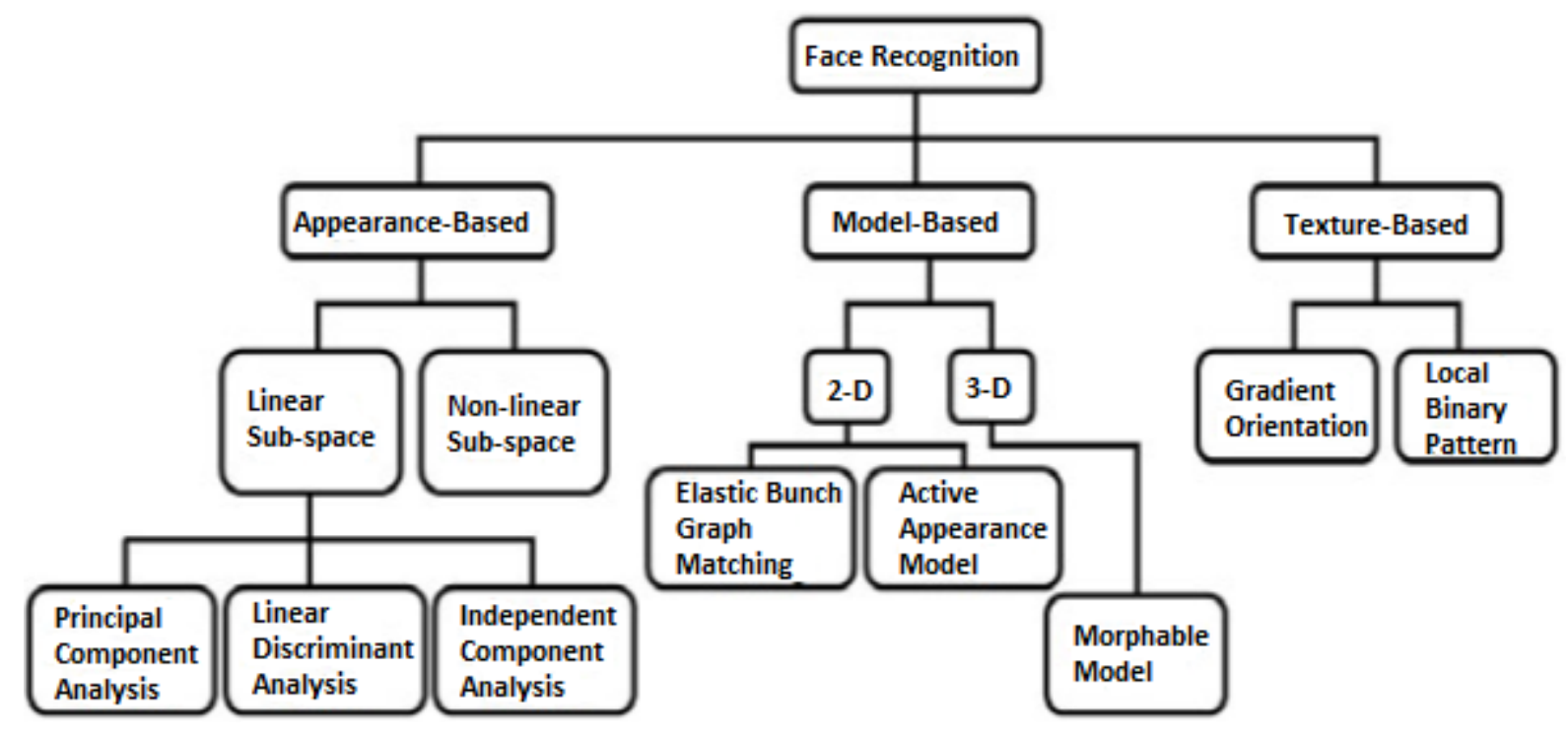

Figure 2.4: Classification of face recognition techniques [24, 39] 
- Appearance-based methods. This approach is based on two-dimensional images. In this technique, the whole face region from an acquired face image is used to generate a compact structure by mapping a high-dimensional image into a low-dimensional subspace [24]. A subspace is the representation of basic vectors which are derived using a training set of images. Commonly used mapping techniques are Principal Component Analysis (PCA), Linear Discriminant Analysis (LDA) and Independent Component Analysis (ICA).

- Model-based methods. This method was built based on three-dimensional face models along with two-dimensional images. Face bunch graphs (FBG) and Active Appearance model (AAM) are examples of this approach.

- Texture-based methods. This is an approach in which local features which are robust to variations in pose and illumination are used. Local Binary Patterns (LBP) is an example of this type of method.

\subsubsection{Appearance-based Approaches}

In an appearance based approach, the face is represented as set of raw intensity images and each image reflects a high-dimensional vector. Then statistical techniques are applied in order to extract a feature space from the image distribution and the sampling image is compared against the training data set. Appearance based methods can be categorized into linear and nonlinear types. Linear appearance-based method implements linear dimensional reduction. The coefficients of projected face vectors represents features of each face image. On the other hand, the non-linear appearance-based method is highly complex. This approach is a complex version of linear appearance based methods [49].

\section{(i) Principal-Component Analysis}

Principal Component Analysis also known as eigenfaces is among the most prominent statistical methods $[5,6]$ which uses minimum mean squared error linear subspace. This is a mathematical procedure that performs dimensionality reduction on extracting principal components of a multidimensional data. Eigenfaces are two-dimensional, shadowlike grayscale faces as shown in the figure 2.5 [40] from which feature vectors are derived.

The first principal component i.e., the first coordinate system is the linear combination of the actual dimensions that has highest variability [7]. The $\mathrm{m}$-th principal component is the linear combination 
having maximum variability and orthogonal to $\mathrm{m}-1$ first principal components. The $\mathrm{m}$-th coordinate will be in the direction of (m-1) principal component with maximum variance. With the help of this technique, actual data can be reduced to up to $1 / 1000^{\text {th }}$ of its size which is a major advantage [40]. But PCA is compatible only with full frontal images which is one of its drawbacks. Also, it is less robust to changes in facial features i.e., due to accessories or due to variations in illumination.

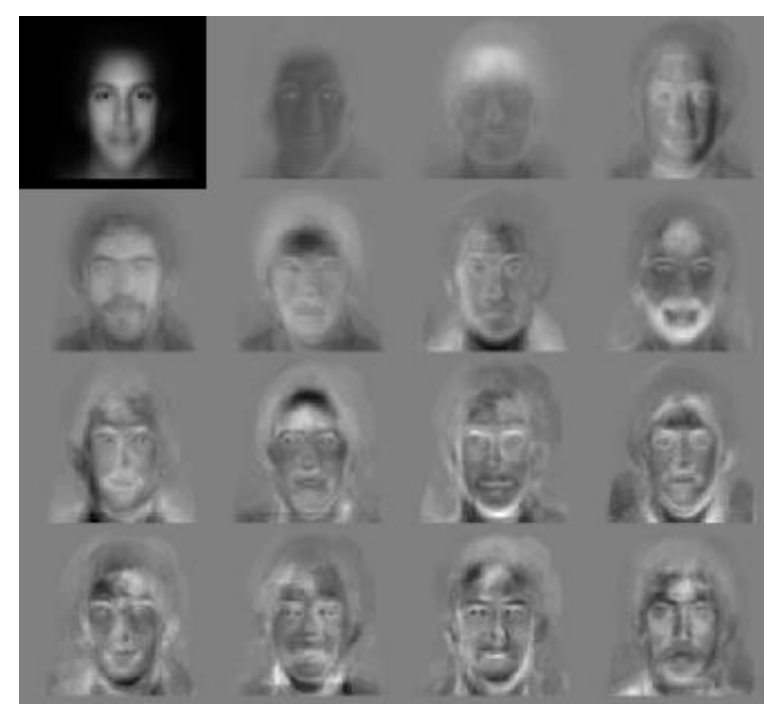

Figure 2.5: Eigen faces

\section{(ii) Linear Discriminant Analysis}

The main idea behind LDA is to find linear combinations of features as well as maintaining separation between classes unlike PCA. Typical LDA is designed considering only two classes. However, multi-class projection vectors are more commonly used. In LDA, data points from different classes should be farther apart while those in same class can be close. LDA obtains different projection vectors for different classes [9]. LDA is predominantly used where an accelerated matching process is desired. This is a statistical method which is resistant to variations in lighting and expressions making it advantageous over PCA. However, it is not capable of dealing with non-frontal images.

Similar to PCA, LDA can efficiently retain only a certain amount of data sufficient to accomplish its matching task. Still, it demands larger datasets to make use of a large amount of reference data for matching. The necessity of larger databases is not always desirable in real time applications. 
On testing the AR database $[8,10]$ using PCA and LDA face recognition, they were able to show that PCA outperforms LDA if the training set is small. The objective function of LDA is given by Cai.et.al [50] from the reference section.

\section{(iii) Independent Component Analysis}

As the name says, the goal of Independent Component Analysis is to convert data to a linear combination of statistically independent data points, thus providing independent rather than uncorrelated image representation. ICA which a more powerful data representation- as an alternative to PCA [51].

\subsubsection{Model-based Approaches}

Model-based approaches try to model a human face sufficient to recognize an image. Model-based techniques enable matching of face images across a range of poses by deriving pose-independent facial images [48]. This requires detection of numerous landmark points on the face such as the corners of the mouth and eyes, the tip of the nose, the chin etc. Unlike appearance-based techniques, model-based ones use multiple landmarks making this a much more reliable technique. Also, generation of realistic animation of faces is possible with these approaches [24].

\section{Elastic Bunch Graphs (EBGM)}

Elastic bunch graph matching is a model-based technique which deals with nonlinear aspects such as variations due to illumination, pose and expression. In this method, Gabor wavelets are used to label each node of a graph $[14,24]$ with set of Gabor coefficients. These coefficients are extracted using a Gabor Kernel containing five different spatial frequencies and eight different orientations. A face bunch graph can be modeled in two phases. In the first phase, the user has to manually mark the required landmarks and define the geometric structure of image graph of first image. This image graphs helps the remaining images in the training set to semi-automatically develop their respective image graphs. In the second phase, a face bunch graph is obtained from a set of corresponding image graphs. Therefore, each node in a FBG is marked with a set of Gabor coefficients. A set of these coefficients at each landmark point is termed as a "bunch" [7, 24]. See Figure 2.6 [39] for the EBGM of a face map.

An edge between the FBG is assigned based on average distance between corresponding nodes in the training set [24]. Landmark points for a new image are obtained by maximizing the similarity 
between the FBGs of alike poses [7]. This process is known as Elastic Bunch Graph Matching (EBGM). EBGM suits images which are not neutral, however, the accurate marking of landmarks plays a key factor.

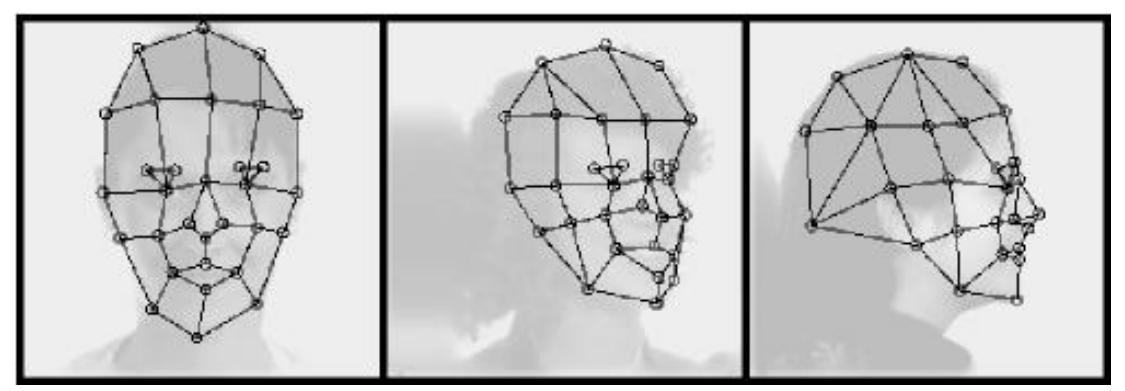

Figure 2.6: EBGM of a face [39]

\subsubsection{Texture-based Approaches}

In appearance-based techniques, raw pixel intensity values- which are quite vulnerable to variations in lighting, occlusions and facial expressions- are used in extracting facial features. Texture-based techniques, which are designed based on distribution of local pixel intensities are an alternative to address this issue. The Local Binary pattern approach is a well-known example of such a technique.

\section{Local Binary Patterns (LBP)}

Local Binary Patterns (LBP) have been effectively implemented to derive local intensities in several image retrieval, segmentation and texture classification applications. The image pixels of a $3 X 3$ neighborhood or region are used in extracting these LBP features [24].

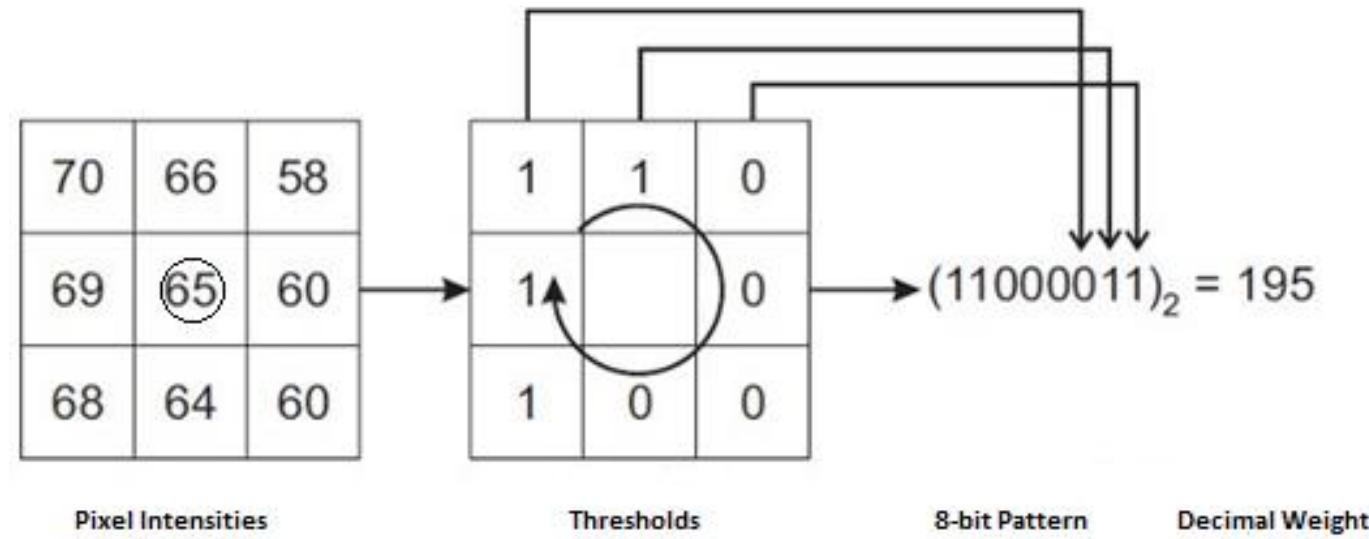

Figure 2.7: Sample illustration of LBP [42] 
The basic LBP operator compares the 8 neighboring pixel intensity values against central pixel intensity in a region and represents the output as an 8-bit binary strings, - [41] which is further converted into a decimal number by applying weights to each bit [24] as shown in figure 2.7.

Multiscale LBP is an extension to basic LBP. MLBP introduces a radius parameter ' $R$ ', which means the remaining pixels are at a distance of ' $R$ ' from the center pixel [24]. Sampling points 'P', stay along the circle of radius ' $R$ '. An MLBP operator with radius ' $R$ ' and sampling points ' $P$ ' is often represented as $L B P_{P, R}[41]$.

Following LBP encoding of each pixel, a facial image is further divided into numerous small windows and the histogram of LBP in each window is calculated. The number of bins in the histogram equals 8 and $2^{P}$ in LBP and MLBP respectively [24].

\subsubsection{Comparison of various feature extraction techniques}

In this section, let us summarize the methods [24, 41], key factors, advantages and disadvantage of each category. Refer to Table 2.1 .

Table 2.1: Summary of Feature Extraction Techniques

\begin{tabular}{|c|c|c|c|c|}
\hline Technique & Methods & Features & Advantages & Disadvantages \\
\hline $\begin{array}{c}\text { Appearance- } \\
\text { based }\end{array}$ & $\begin{array}{c}\text { PCA, LDA, } \\
\text { ICA }\end{array}$ & Eyes and Mouth & $\begin{array}{c}\text { Higher recognition } \\
\text { rates } \\
\text { Model-based }\end{array}$ & $\begin{array}{c}\text { Need large data sets } \\
\text { of high quality } \\
\text { images with proper } \\
\text { illumination }\end{array}$ \\
\hline EBGM & $\begin{array}{c}\text { Corners of the } \\
\text { mouth and eyes, } \\
\text { tip of nose, chin } \\
\text { etc. }\end{array}$ & $\begin{array}{c}\text { Robust to } \\
\text { illumination, pose } \\
\text { and expressions } \\
\text { and } \\
\text { Texture-based }\end{array}$ & $\begin{array}{c}\text { Needs longer } \\
\text { high accuracy rates }\end{array}$ & $\begin{array}{c}\text { computational time } \\
\text { Distribution of } \\
\text { local pixel } \\
\text { intensities in an } \\
\text { image }\end{array}$ \\
$\begin{array}{c}\text { Invariant to } \\
\text { illumination and } \\
\text { spatial rotations, } \\
\text { Highly accurate }\end{array}$ & $\begin{array}{c}\text { Binary data is } \\
\text { sensitive to noise, } \\
\text { miss some local } \\
\text { patterns due to } \\
\text { non-consideration } \\
\text { of center pixel }\end{array}$ \\
\hline
\end{tabular}




\subsection{Statistical Distance Measures}

Usually in probability theory or information theory, statistical distance measure is used to qualify the performance of two statistical objects such as random samples or probability distributions etc. In this thesis we used a couple of statistical measures to calculate distance between two probability distributions.

\subsubsection{Kullback-Leibler Divergence}

Kullback and Leibler introduced an information measure, termed as Kullback-Leibler divergence or relative entropy in 1951, to assess the distance between two distributions [45]. Let us assume two probability distributions $f(x)$ and $g(x)$ for a random variable $X$. Kullback-Leibler divergence or relative entropy is given as [43]:

$$
K L D(f \| g)=D(f \| g)=\sum_{x \in X} f(x) \log \frac{f(x)}{g(x)}
$$

The KL divergence uses the same random variable to compare entropy of two distributions. In this thesis, the Kullback-Leibler divergence measure was used to compare two probability mass functions in order to yield distance scores. This is an asymmetric measure [44].

Kullback-Leibler divergence results in a non-negative score with the minimal score of ' 0 ' when $f$ is the same as $g$, interpreted as no information loss when $g$ is approximated with $f$. Thus, one can conclude that the smaller or closer the value of $D(f \| g)$ to is ' 0 ', the closer the model $g$ is to the true distribution.

This divergence is possible only if both $f(x)$ and $g(x)$ are both non-zero, or else it returns negative infinity. Also, it is necessary to make sure that both the vectors $f(x)$ and $g(x)$ are of the same size to carry out the process. If they are of different sizes, zeroes can be padded to balance their sizes. As this divergence is asymmetric, a robust algorithm calculates the distance scores in both the directions and returns the mean of the scores [44].

The following section lists the properties of Kull-back Leibler Divergence [43, 44, and 45]. 


\subsubsection{Properties of Kullback-Leibler Divergence}

(i) Kullback-Leibler divergence is non-symmetric

$$
D(f \| g) \neq D(g \| f)
$$

This divergence is symmetric if and only if $D(q \| p)=\infty$

(ii) Divergence is always non-negative

$$
D(f \| g) \geq 0
$$

(iii) Divergence equals ' 0 ' if and only if $f(x)=g(x) \quad \forall \quad x \in x$

$$
D(f \| g)=0
$$

(iv) Mutual information is the KL divergence between $f(x, y)$ and $f(x) f(y)$

$$
I(X ; Y)=D(f(x, y) \| f(x) f(y))
$$

Jeffery's divergence (JD) is the symmetrized version of Kullback-Leibler Divergence. Assume two probability distributions $f(x)$ and $g(x)$ for a random variable $X$. Jeffery's divergence is defined as [56, 57]:

$$
J D(f \| g)=D(f \| g)+D(g \| f)
$$

It ranges between ' 0 ' and positive infinity.

\subsubsection{Jensen-Shannon Divergence}

While Kullback-Leibler Divergence is a classic method of finding similarity between two probability distributions, Jensen-Shannon Divergence (JSD) is smoothened version of KLD with some prominent modifications. It is also called as information radius or total divergence from the average [54].

Let us consider we have two match score distributions $f(x)$ and $g(x)$ for a random variable $X$. Jensen-Shannon Divergence (JSD) can be computed using [54]

$$
J S D(f \| g)=\frac{1}{2} D(f \| h)+\frac{1}{2} D(g \| h)
$$

whereh $=\frac{1}{2}(f+g) ; D(f \| h)$ and $D(g \| h)$ are defined as in equation 2.1 
Certain characteristics of JSD make it an appropriate measure over standard measures of information theory such as mutual information or relative entropy [55] etc. Some of them are

- It is a symmetrical measure

- It is a finite measure

- It can be employed over more than two distributions

- It is bounded

- It has adequate branching property

All these aspects constitutes to consider JSD is an advantageous measure over classic ones.

\subsubsection{Properties of Jensen-Shannon Divergence}

JSD is always non-negative. The value of JSD equating ' 0 ' is considered ideal as it says the two distributions are similar.

JSD is a symmetric measure and is bounded. Depending upon the choice of logarithm by the user, the bounds of JSD varies as in equations 2.8 and 2.9 [55].

For a base 2 logarithm, JSD lies between 0 and 1 i.e.

$$
0 \leq J S D(f \| g) \leq 1
$$

Similarly, for a base $e \operatorname{logarithm}$ or natural $\log$, JSD lies between 0 and $\ln (2)$ i.e.

$$
0 \leq J S D(f \| g) \leq \ln (2)
$$

Logarithm of base other than the specified ones yields a different upper bound.

\section{Relation between Statistical Distance Measures}

According to T.Yamano [57], inequality between Jeffery's and Jensen Shannon Divergence is given by

$$
J S D(f \| g) \geq \frac{1}{4} \ln \left(1+J D(f \| g) \quad ; \text { when } f_{i} \geq g_{i}\right.
$$

According to some authors, symmetrized average of KLD or relative entropy is half the value defined by Jeffery [56].

$$
K L D(f \| g)=\frac{1}{2} J D(f \| g)
$$




\subsection{Receiver Operating Characteristics (ROC)}

It is important to determine whether or not a biometric system can be employed for a particular operational environment such that it meets the requirements of optimal performance and to evaluate the methodologies used in biometrics [40]. Receiver operating characteristics have been extensively used for performance evaluation in the field of biometrics.

In general, a receiver operating characteristic graph is a technique used to visualize and select a classifier based on its performance. ROC have been used in signal detection theory which is further extended in field of medicine for decision making [47]. In biometrics, ROC graphs can used to compare the performance among matchers or among subcategories in a database.

ROC is a curve that plots the genuine acceptance rate (GAR) on y-coordinate with respect to false acceptance rate (FAR) on x-coordinates. A test which is faultless or one with high accuracy passes through the upper left corner. In an ROC, area under curve (AUC) is an attribute used to measure the performance and determine the conclusions in comparing methods. Higher the AUC, better is the performance [47]. This is measured on a scale between 0 and 1 . AUC of 1 represents an ideal performance and value $\leq 0.5$ represents worst performance.

\subsubsection{Properties and Uses of an ROC}

An ROC demonstrates following properties [47]:

- Demonstrates the tradeoff between GAR and FAR. An increase in GAR is associated with a decrease in FAR and vice versa.

- The nearer the curve to top left corner, more is the accuracy of the test.

- The nearer the curve to the diagonal of ROC space, worst is its performance.

- Area under curve is an attribute that determines accuracy.

The ROC may be use to [47]:

- Determine the cutoff point at which optimal GAR and FAR are achieved.

- Assess the accuracy of a test.

- Compares the performance among multiple tests. 
Another attribute considered in estimating the accuracy of a test is through determination of Equal Error Rate (EER). The EER is an operating point on ROC where false acceptance rate equals the false rejection rate [46]. Lower the measure of EER, higher is the accuracy of the test. Thus, it is always desirable to have lower error rates.

In practice, comparing the performance of two matchers is a task that is dealt using ROC in biometrics. Most biometrics cannot guarantee that both false acceptance and false rejection rates are low [46]. Thus,

- Secure application needs low FAR

- Convenient application needs low FRR. 


\section{Chapter 3}

\section{Methodology}

West Virginia University has been involved in collecting several large-scale multimodal biometric data collections, with an aim to create datasets that can be used both by government and for academic patterns of FBI. Several series of collection projects were undertaken by WVU since 2008 till date. For this thesis, the NeuroTechnology MegaMatcher SDK v5.0 was used to generate genuine and impostor score distributions for neutral faces from the WVU's 2012 BioCOP collection.

\subsection{Data Acquisition}

Facial images were acquired under controlled conditions using a Canon EOS 5D Mark II digital camera with a Canon Electro-focus (EF) 70-200mm f/2.8L Image Stabilization (IS) II Ultrasonic Motor (USM) telephoto zoom lens. Images were captured twice in each session and two sessions of data were collected from some participants at least separated by two months. Captured images include standard 5-pose face capture, i.e., $0^{\circ}, \pm 45^{\circ}, \pm 90^{\circ}$ along with two frontal images each with color card and random identification number respectively. All images were captured at 2 meters against a neutral gray background with three-point tungsten lighting used to eliminate hotspotting and shadows. However, matching experiments were performed only on frontal pose images ranging from 8 to 16 images per participant based on number of sessions. Images of 1200 participants were captured during this collection covering subjects from a wide range of demographics. Parameters such as spatial resolution, focal length and exposure time etc. for all images were maintained uniform throughout the collection process.

A total of 13976 neutral pose images comprising of 'RAW' and 'SAP', colored and numbered card images were used in this study. While 'RAW', colored and numbered card images were used as they were captured, Subject Acquisition Profile (SAP) images (SAP 50 and SAP 51) are processed face images of 'RAW' version whose size and position are cropped according to ANSINIST standards [36]. All SAP 50s specifies the 'head and shoulder' composition requirements while SAP51s specifies 'head only' composition requirements [36].

Table 3.1 lists the specifications of images used for this work. 
Table 3.1: Specifications of Images in the Database

\begin{tabular}{|c|c|}
\hline Image Format & JPEG \\
\hline Aspect Ratio (other than SAP) & $3: 2$ \\
\hline Size of RAW Image & $6 \mathrm{MB}$ \\
\hline Back Ground & Neutral Gray \\
\hline Spatial Resolution & $72 \mathrm{dpi}$ \\
\hline Exposure Time & $1 / 60 \mathrm{sec}$ \\
\hline Focal Length & $135 \mathrm{~mm}$ \\
\hline Color Representation & sRGB \\
\hline Aspect Ratio (SAP) & $3: 4$ \\
\hline RAW image resolution & $5616 \times 3744$ \\
\hline Cropped SAP 50 image resolution & $3300 \times 4400$ \\
\hline Cropped SAP 51 image resolution & $2400 \times 3200$ \\
\hline
\end{tabular}

The metadata acquired during this collection process includes information about participant's age, gender, ethnicity, colors of hair and iris, facial hair, height and weight etc. This information enabled us in categorizing the data demographically.

The human face contains a variety of information for adaptive social interaction with people. Human faces provide demographic information such as (i) Gender (ii) Age and (iii) Ethnicity. These demographics are involved in human facial perception and recognition. In order to explore demographic characteristics, the entire database of 1200 subjects was further categorized into subcohorts for each demographic.

Gender recognition can be considered as an extended corollary to facial detection. Nowadays, more research is being performed on the gender classification aspect which can considerably impact the distribution of match scores. Facial features of subjects of the same gender most likely possesses more of similarities compared to their gender than when matched against the opposite gender. One of the most important things considered while classifying gender is facial hair. We have 705 male and 495 female participants in this data collection.

Age is also a vital demographic in its own way. In most cases, one can estimate a person's age approximately. The major facial characteristic that can impact age is wrinkled skin. Three such cohorts were considered to analyze the impact of age on distribution of match scores. Major share 
of participants i.e. 886 were between ages 20-30 while 113 belong to age 31-49 cohort and 59 fall between ages $50-70$.

People belonging to the same ethnic group share culture, language and region etc. along with a considerable amount of facial features. These facial features may affect the distributions of match scores. In our current database, we have participants from various ethnicities such as African, African-American, Asian, Asian-Indian, Caucasian, Hispanic, Middle Eastern and Pacific Islanders. A tiny subset of subjects other than the mentioned ethnicities were placed in 'Others'. Among all, there are 727 Caucasians while there are 20 Africans, 76 African-Americans, 105 Asians, 137 Asian-Indians, 56 Hispanic, 61 Middle-Eastern, 4 Pacific Islanders and 14 in 'Others'.

\subsection{Commercial Matching Software}

Many biometric companies in the present market are offering their own customized commercial face recognition matching software. One of the best available facial matching SDKs is NEC-Neo Face Suit. But NEC is available exclusively for law enforcement agencies or other federal organizations. Some of the commercial FR matchers and their tasks are listed in table below [60].

Table 3.2: Commercial Matching Software and their Tasks

\begin{tabular}{|c|c|c|}
\hline FR Matchers & Tasks & Website \\
\hline NEC Neo Face Suit & $\begin{array}{l}\text { Facial image matching; CCTV watch list } \\
\text { screening; searching archived videos }\end{array}$ & http://nec.com \\
\hline Cognitec Face VACS & $\begin{array}{l}\text { Face verification/ identification; } \\
\text { enrollment from a video to track an } \\
\text { individual }\end{array}$ & $\begin{array}{l}\text { http://cognitec- } \\
\text { systems.de }\end{array}$ \\
\hline L1 Face IT SDK & $\begin{array}{l}\text { Face identification; Multi-modal } \\
\text { recognition }\end{array}$ & http://www.11id.com \\
\hline $\begin{array}{c}\text { Neurotechnology’s } \\
\text { VeriLook }\end{array}$ & $\begin{array}{c}\text { Face verification/ identification; } \\
\text { enrollment from a video to track an } \\
\text { individual }\end{array}$ & $\begin{array}{l}\text { http://neurotechnology.c } \\
\text { om }\end{array}$ \\
\hline Animetrics Face R & $\begin{array}{c}\text { Face verification/ identification; } \\
\text { enrollment from a video to track an } \\
\text { individual; Multi-modal recognition }\end{array}$ & http://animetrics.com \\
\hline Google Inc.'s PittPatt & $\begin{array}{l}\text { Face verification/ identification; } \\
\text { enrollment from a video to track an } \\
\text { individual }\end{array}$ & http://pittpatt.com \\
\hline $\begin{array}{l}\text { Genex Technologies Sure } \\
\text { Match 3D Suite }\end{array}$ & Face identification & http://genextech.com \\
\hline Face-TEK's Notiface II & Face identification & http://face-tek.com \\
\hline Acsys Biometrics & $\begin{array}{l}\text { Face verification/ identification; } \\
\text { enrollment from a video to track an } \\
\text { individual }\end{array}$ & $\begin{array}{c}\text { http://acsysbiometrics.co } \\
\text { m }\end{array}$ \\
\hline
\end{tabular}


For this work, Neurotechnology's Mega Matcher multi-biometric SDK was chosen because it is readily available for purchase by academic institution. Unlike many other commercial matchers it is more affordable.

\subsubsection{Mega Matcher Matching Software}

NeuroTechnology developed a multimodal biometric SDK that covers wide range of applications in order to meet the increasing demand of authentication. The components includes VeriFinger, VeriEye, VeriLook and VeriSpeak for fingerprint, iris, facial and vocal identification respectively. The MegaMatcher SDK is intended for the development of large-scale automated fingerprint identification and multi-biometric systems. All these components are available in either the Standard version which is suitable for PC based applications or Extended version which includes a ready to use matching server.

The illustration below demonstrates the relation between various products supported by the NeuroTechnology Biometric SDK. According to NeuroTechnology this "large SDK product" is composed of several "smaller SDKs" [53]. The MegaMatcher standard version is an embedded component of MegaMatcher extended version where the MegaMatcher Standard SDK is composed of all Extended SDKs of VeriFinger, VeriEye, VeriLook and VeriSpeak which are in turn includes the components of the Standard SDKs of VeriFinger, VeriEye, VeriLook and VeriSpeak respectively.

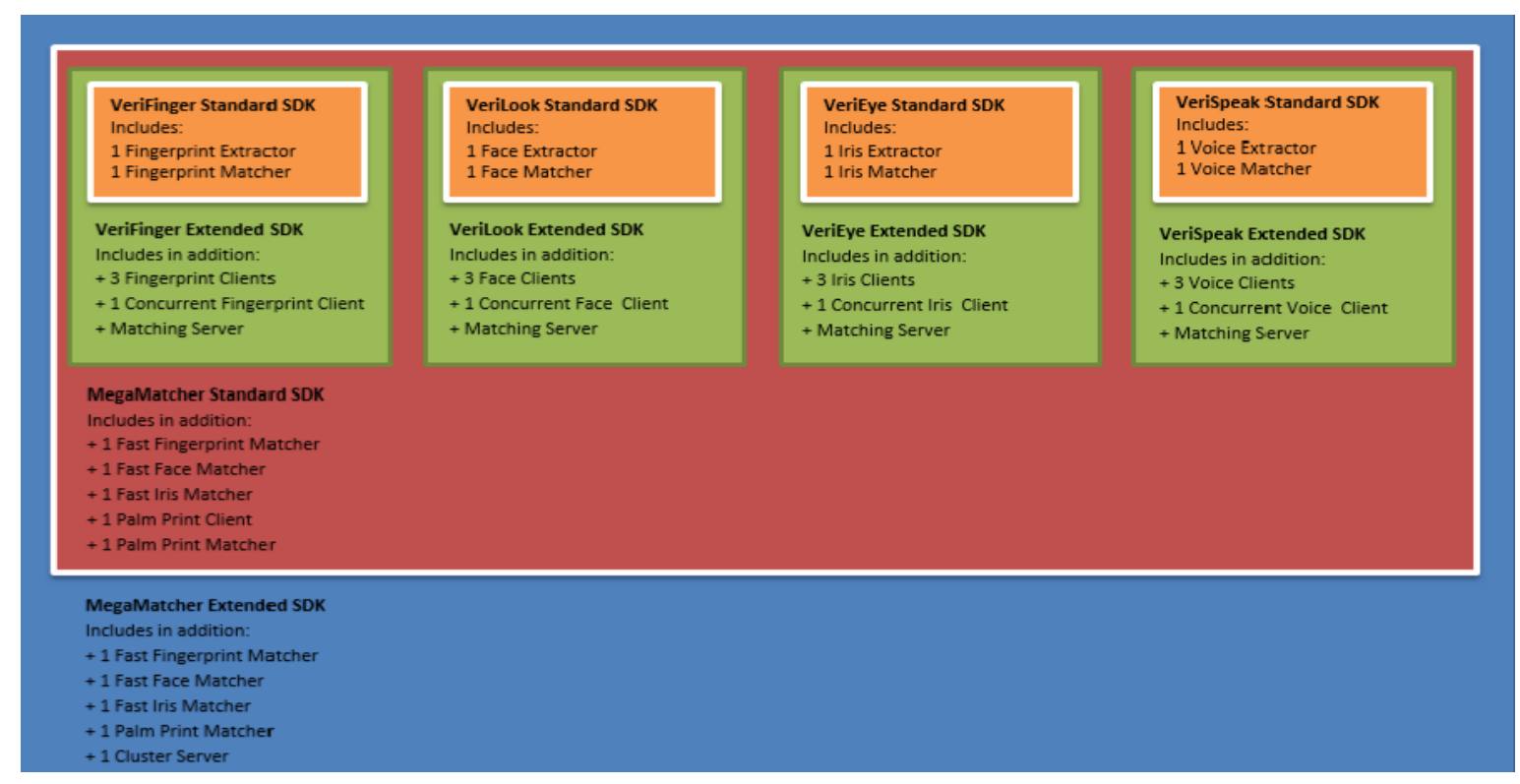

Figure 3.1: Schema of NeuroTechnology Biometric SDK [53] 
The MegaMatcher product is based on MegaMatcher scalable multi-biometric technology. As discussed earlier, MegaMatcher technology is designed to meet requirements of multi-biometric system developers where reliability and speed of biometric identification while dealing with large databases is a major concern. High productivity and efficiency are seen in fused algorithms containing face, iris, fingerprint, voice recognition engines. These engines can be used individually or can be fused with the help of integrators for improved identification results. Thus, MegaMatcher is suitable not only for civil applications but also for forensic applications.

The fast parallel matching is achieved with the help of fault-tolerant scalable cluster software. Additional tasks such as the processing of large numbers of identification requests and handling large databases can be performed using this cluster software whereas server software for local biometric systems was included in MegaMatcher. For swift development of client side software, Java and .Net components were incorporated in MegaMatcher.

The Fig 3.1 demonstrates that MegaMatcher is composed of several subcomponents organized such that each of them handles one particular modality. For our work, the VeriLook component of MegaMatcher fulfills our requirements. Facial recognition technology is being widely used in various systems including physical access control and computer user authentication.

VeriLook facial identification technology offers fast and reliable identification with live face detection, an ability to process multiples faces in a frame. In general, face matching is performed using certain facial features extracted by these systems. It is capable of fast face matching in both verification and identification modes. VeriLook exists in two versions, namely the standard and extended versions. For this work, standard version of VeriLook was used.

VeriLook includes the following Components:

- Face Extractor: creates face template from face images

- Face Matcher: performs template matching in verification and identification modes

- Face Client: combination of Face Extractor and Face BSS

- Face (BSS): Biometric Standards Support allows to support additional face templates and image format standards

A typical face identification system developed with VeriLook standard version can be illustrated using the following client work station data flow diagram. See figure 3.2. 


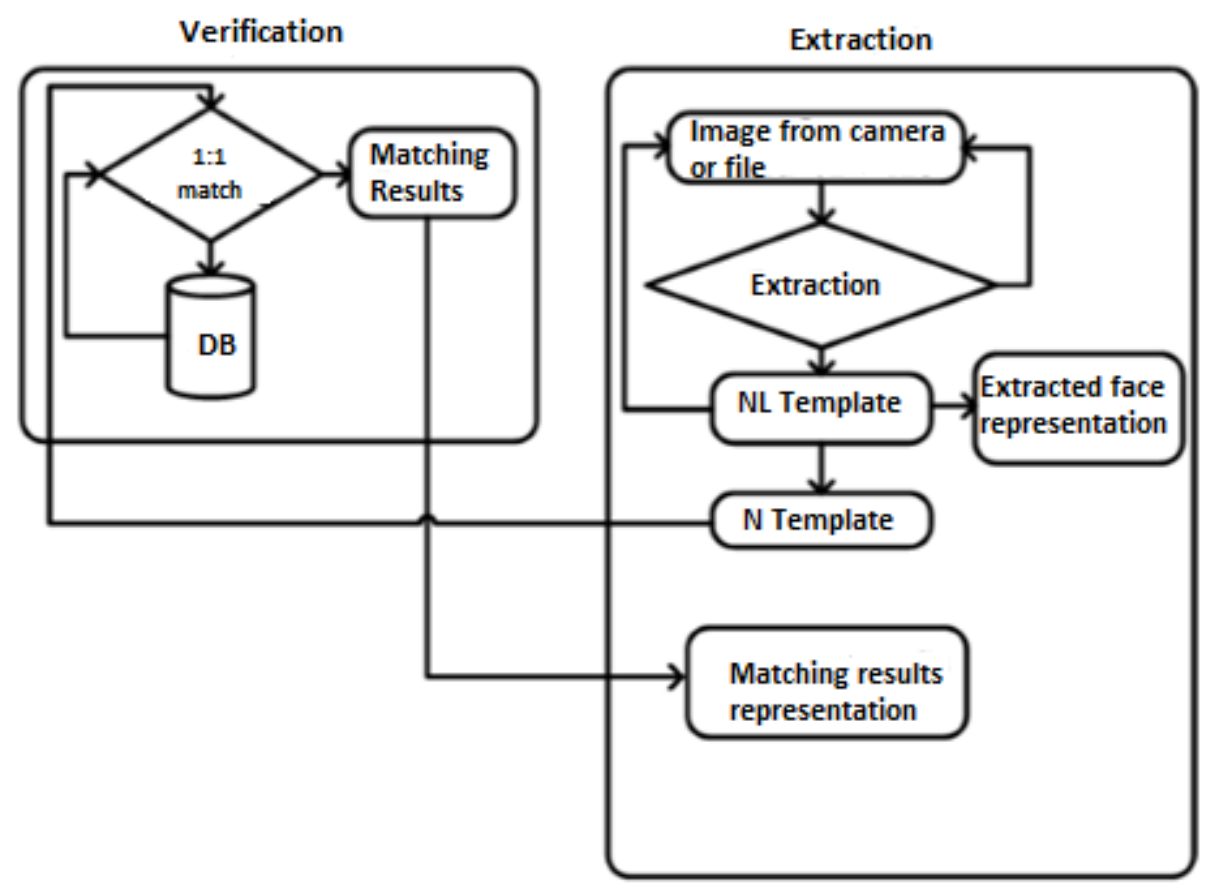

Figure 3.2: Data Flow diagram of application based on VeriLook Standard SDK [53]

Matching is carried out in two steps: Extraction and Identification or Verification.

\section{Algorithm:}

- Step 1: Raw images from the camera or pre-existing images from a database can be given as input

- Step 2: Templates for raw images are created at the extraction phase. NL Template is term used to denote one face template.

- Step 3: Face Extraction is carried out for all images in database and set of these created templates is termed as $\mathrm{N}$ Template

- Step 4: Matching is performed on these 'NTemplates' in either identification mode or verification mode.

- Step 5: Matching results are obtained and represented in desired formats.

**NL Template and N Template are the attributes coined for NeuroTechnology SDK.

Since MegaMatcher SDK is a proprietary software, information regarding the source code on which the SDK was designed is kept confidential. 


\subsection{Experimental Setup}

MegaMatcher v5.0 SDK and its sub components supports JAVA projects which are built and managed using Eclipse, a Java IDE and Apache's Maven, a build automation tool used primarily for Java projects. Using this environment, a customized Java script was written to perform the matching task along with the acquisition of quality scores. Attributes used in our customized script are listed in Table 3.3 [53] and Table 3.4 [53] lists the properties of biometric engine.

Table 3.3: Some of the Attributes in NeuroTechnology SDK

\begin{tabular}{|c|c|}
\hline Attribute & Description \\
\hline NBiometricOperation & $\begin{array}{l}\text { A part of NBiometricEngine used in } \\
\text { providing high level operations such as } \\
\text { template creation, enrollment, } \\
\text { identification or verification }\end{array}$ \\
\hline NBiometricStatus & Enumerates biometric status values \\
\hline NBiometricTask & $\begin{array}{l}\text { Contains functions which create and } \\
\text { modify biometric engine tasks such as } \\
\text { adding new subject or deleting existing } \\
\text { subject, retrieving biometric operation } \\
\text { and calculating statistics of from specific } \\
\text { task etc. }\end{array}$ \\
\hline NFace & $\begin{array}{l}\text { A function for biometric engine to deal } \\
\text { with face template. These functions } \\
\text { include creating new face object, retrieve } \\
\text { the face image of specified NFace object }\end{array}$ \\
\hline NMatchingSpeed & Defines rate of matching. \\
\hline NMatchingResult & $\begin{array}{l}\text { Retrieve information such as biometric } \\
\text { connection, ID, match score, subject etc. } \\
\text { of a specific matching result. }\end{array}$ \\
\hline NSubject & $\begin{array}{l}\text { Represents biometric information such } \\
\text { as template, matching results etc. related } \\
\text { to a person. }\end{array}$ \\
\hline NBiometricClient & $\begin{array}{l}\text { It provides biometric connection } \\
\text { function for biometric engine. Also } \\
\text { provides function for biometric data } \\
\text { capture and its transfer through various } \\
\text { connections }\end{array}$ \\
\hline NLicense & $\begin{array}{l}\text { Provides functionality for getting and } \\
\text { releasing license and internal licensing } \\
\text { service. }\end{array}$ \\
\hline
\end{tabular}


Table 3.4: Matching Properties of Biometric Engine

\begin{tabular}{|c|l|}
\hline Properties & \multicolumn{1}{|c|}{ Description } \\
\hline Matching.MaximalResultCount & $\begin{array}{l}\text { Allows maximal possible result } \\
\text { number i.e. maximal matching } \\
\text { iterations }\end{array}$ \\
\hline Matching.Threshold & $\begin{array}{l}\text { Defines the matching threshold. } \\
\text { Matching scores below this will be } \\
\text { ignored. }\end{array}$ \\
\hline
\end{tabular}

Initially we specified the usage format of probe and gallery images along with license version of the SDK. Templates are created for each image upon its creation whenever the NBiometricStatus flag says ' $O K$ ' followed by enrollment of each subject. A minimum of 0.08- 0.21 seconds is required for face template creation. Biometric components for extraction and matching of face can be achieved using Biometrics.FaceExtraction and Biometrics.FaceMatching. In order to reduce the probability of missing matches, a matching threshold of ' 0 ' was used i.e. $100 \%$ false acceptance rate. Matching speed was chosen as low and MaximalResultCount helps in accommodating maximum possible subjects.

With all these specifications Match Scores were generated and stored in .csv files. Generated scores were further used in plotting genuine and impostor graphs. Also, quality scores were generated using getQuality () attribute. However, to date, no efforts were made to assess quality scores.

\subsection{Matching Procedure}

Matching is done through the comparison of templates with an aim to check if they are of the same person's. The result of such comparison is a similarity score. The higher the score, the higher is the probability that the collected features belongs to right person. Templates can be complex having information of multiple modalities and multiple records (a basic unit of a template) of a modality. Matching is performed on these records and depending on the modality, the resulting scores can either be fused in order to return an ultimate score or consider the available best score as a final score. In face modality, the fused score is returned. 


\subsubsection{Matching Threshold and FAR/FRR}

The strictness of the matching algorithm is controlled by the NMatcher.MatchingThreshold parameter of NMatcher. The matching threshold is a minimum score that verification and identification functions accept to assume that the matched templates belong to same person. The higher the threshold, the more similar feature collections will be required to yield positive results [53]. The matching threshold is in turn related to the FAR and FRR. The higher is threshold, the lower the FAR and higher the FRR and vice versa. Table 3.5 [53] lists the Matching Threshold for NMatcher.

The threshold can also be calculated using the formula:

$$
\text { Threshold }=-12 * \log _{10}(F A R)
$$

Where the FAR is a non-percentage value.

A matching threshold should be selected according to our desired FAR and the system's development requirement. In verification mode, FAR is calculated for single match (1:1) whereas in identification mode (1: N), false acceptance accumulates. Identification false acceptance can be calculated using the formula:

$$
\left(1-(1-F A R)^{N}\right) * 100
$$

where, $\mathrm{N}$ is the size of the database.

Table 3.5: Relation between Matching Threshold and FAR

\begin{tabular}{|c|c|}
\hline FAR & Matching Threshold (score) \\
\hline $100 \%$ & 0 \\
\hline $10 \%$ & 12 \\
\hline $1 \%$ & 24 \\
\hline $0.1 \%$ & 36 \\
\hline $0.01 \%$ & 48 \\
\hline $0.001 \%$ & 60 \\
\hline $0.0001 \%$ & 72 \\
\hline $0.00001 \%$ & 84 \\
\hline $0.000001 \%$ & 96 \\
\hline
\end{tabular}




\section{Chapter 4}

\section{Experimental Results}

\subsection{Face Image Match Score Analysis}

Match Scores were generated using VeriLook component of MegaMatcher. To generate a distribution of genuine match scores, a single image is taken as probe, and all the other images belonging to same participant are treated as gallery in order to generate match scores. The distribution of impostor scores is obtained by choosing an image from one participant and matching it against all other images from rest of the participants in the dataset. For both match genuine and impostor matching experiments, the probe image was not included in the gallery. For impostor matching, the matcher experienced an "out of memory" exception due to large image file size while considering entire dataset for impostor matching. In order to overcome this, a random subset of 2000 gallery images belonging to all 1200 subjects was created for each individual probe image (probe not contained in gallery). Hence, impostor match score distributions were generated for each individual probe images use the small gallery datasets, and these distributions were combined into a total imposter score distribution for each case considered. Figure 4.1 shows of genuine and impostor match scores for face images in the 2012 Biometric dataset. Table 4.1 summarizes the maximum and minimum match scores along with percentage and range of maximum concentration.

Table 4.1: Summary of MatchScores for Overall Data

\begin{tabular}{|c|c|c|c|}
\hline Distribution/Category & $\begin{array}{c}\text { Max. } \\
\text { Matchscore }\end{array}$ & $\begin{array}{c}\text { Min. } \\
\text { Matchscore }\end{array}$ & $\begin{array}{c}\text { Approximate percentage of } \\
\text { maximum concentration }\end{array}$ \\
\hline Genuine & 5730 & 0 & $\begin{array}{c}99 \% \text { of scores are exists in } \\
\text { range of } 0 \text { and } 3000\end{array}$ \\
\hline Impostor & 974 & 0 & $\begin{array}{c}99.9 \% \text { of scores exists in } \\
\text { range of } 0 \text { and } 30\end{array}$ \\
\hline
\end{tabular}

Receiver operating characteristic (ROC) curve was plotted using this data. Attributes such as area under curve and equal error rate were observed as 0.9096 and 0.1367 respectively. Although these scores does not represent $100 \%$ accuracy, considerable accuracy was seen. Refer Figure 4.4 for the characteristic curve of overall data. 


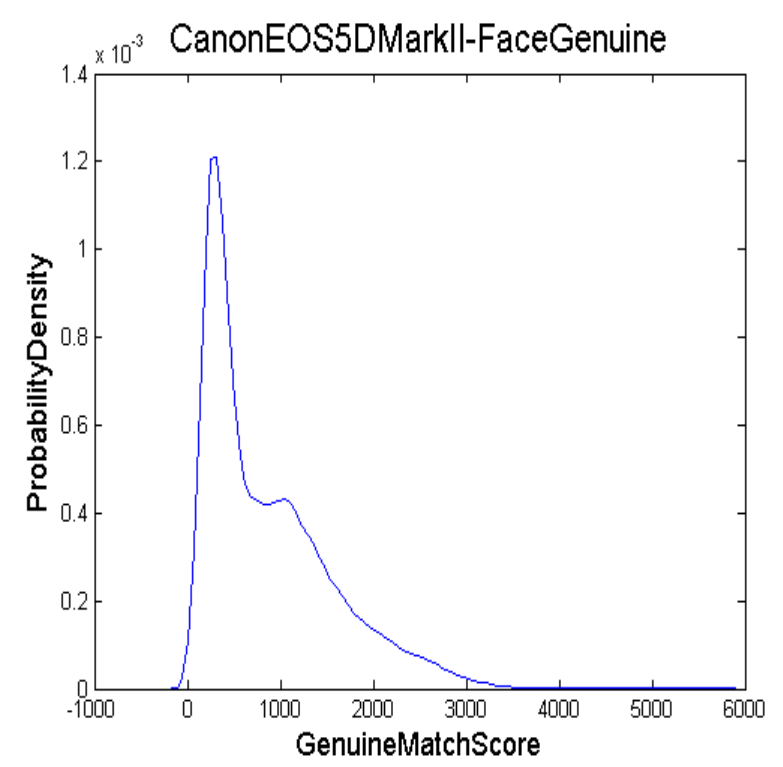

(a)

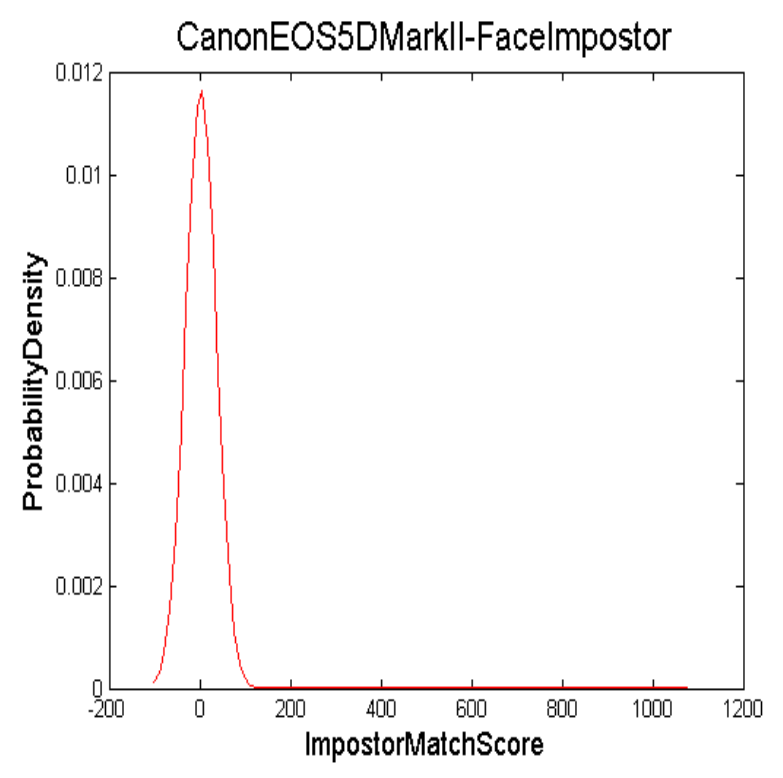

(b)

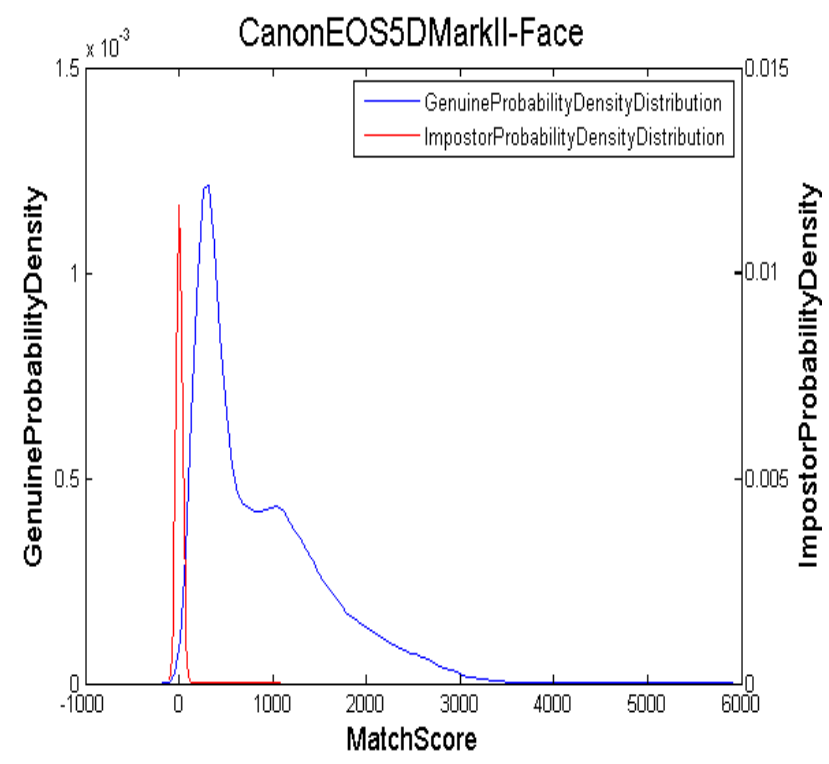

Figure 4.1: Match score distributions for face images from a Canon 5D Mark II digital camera.

(a) Genuine distribution. (b) Impostor distribution. (c) Combined distribution of match scores.

(c) 


\subsection{Demographic Break Down of Face Match Scores}

To explore whether or not demographic characteristics, including gender, age and ethnicity impacted match score distributions, the face data was divided into subsets. A summary of results are provided in the following sections, with a complete listing of genuine and imposter score distributions for section 4.2.3 are provided in Appendix A. Receiver Operating Characteristic (ROC) curves were plotted to determine the performance of overall data against demographics as well as among the demographics. Also, a couple of statistical distance measures were calculated for each demographics from the complete data and scores were tabulated.

\subsubsection{Gender-Based Face Match Score Distributions}

Matching experiments were carried out separately for male \& female and corresponding genuine and impostor match scores were generated. Impostor matching was carried out on a random data subset consisting of 1800 images belonging to male and 1600 images belonging to female to overcome the "out of memory" exception experienced by the matcher (as described above). Sample results are provided in Figures $4.2 \& 4.3$ for female and male face images respectively. A comprehensive list of gender-based face match score are provided in Table 4.2. The genuine match score distributions of the separate male and female cohorts do not show significant differences over the match score distributions of the combined male/female population shown in Figure 4.1 (again, when considering the relative scales of the probability density values on the $y$ axes). The imposter score distribution for females is narrower than males, most likely due to the smaller number of female participants (thus, a smaller number of images) in the data collection.

Table 4.2: Summary of MatchScores for Gender Demographics

\begin{tabular}{|c|c|c|c|c|}
\hline $\begin{array}{c}\text { Type of } \\
\text { Demographic }\end{array}$ & Category & $\begin{array}{c}\text { Max. } \\
\text { Match } \\
\text { score }\end{array}$ & $\begin{array}{c}\text { Min. } \\
\text { Match } \\
\text { score }\end{array}$ & $\begin{array}{c}\text { Approximate percentage of maximum } \\
\text { concentration }\end{array}$ \\
\hline \multirow{2}{*}{ Female } & Genuine & 5521 & 0 & $\begin{array}{c}99 \% \text { of scores ranges between } 0 \text { and } \\
3000\end{array}$ \\
\cline { 2 - 5 } & Impostor & 321 & 0 & $99 \%$ of scores ranges between 0 and 35 \\
\hline \multirow{2}{*}{ Male } & Genuine & 5730 & 3 & $\begin{array}{c}99.5 \% \text { of scores ranges between } 0 \text { and } \\
3200\end{array}$ \\
\cline { 2 - 5 } & Impostor & 558 & 0 & $\begin{array}{c}99.8 \% \text { of scores ranges between } 0 \text { and } \\
40\end{array}$ \\
\hline
\end{tabular}




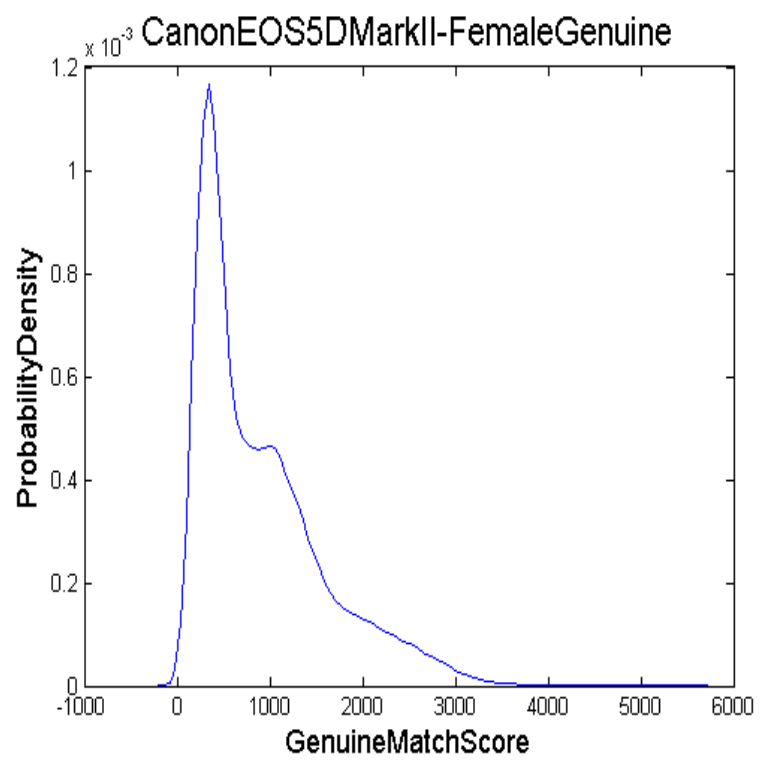

(a)

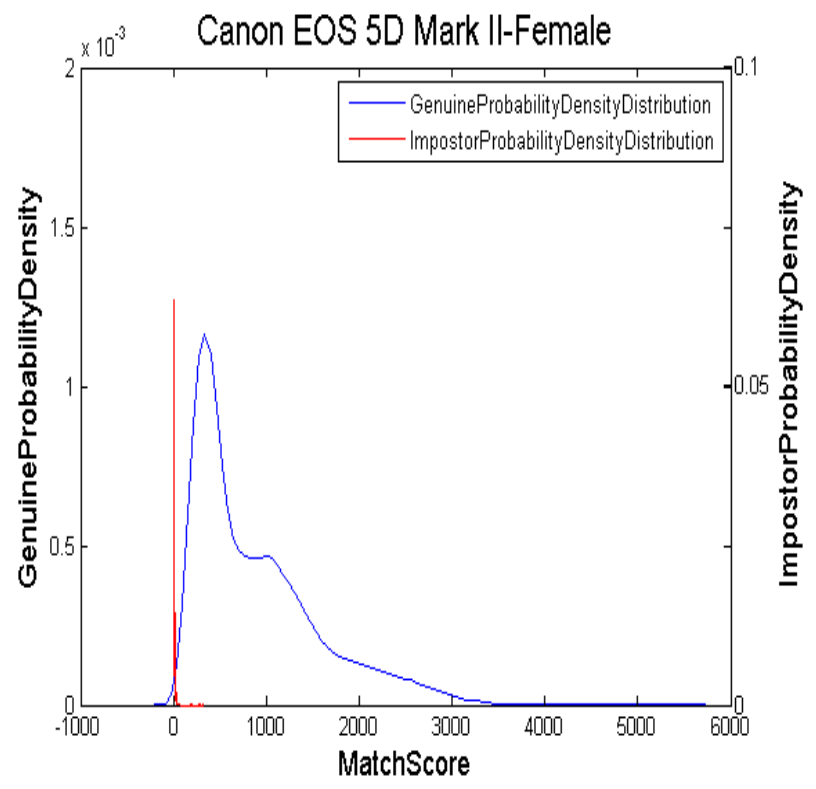

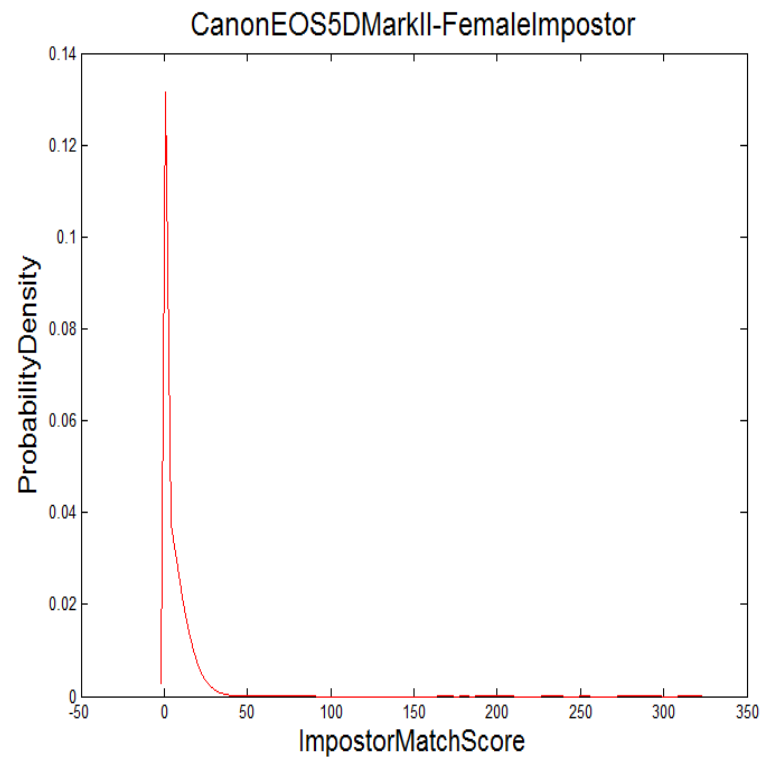

(b)

Figure 4.2: Genuine and imposter score distributions for female face images from Canon EOS 5D Mark II camera. (a) Genuine distribution of female face. (b) Impostor distribution of female face. (c) Combined distribution of match scores of female face.

(c) 


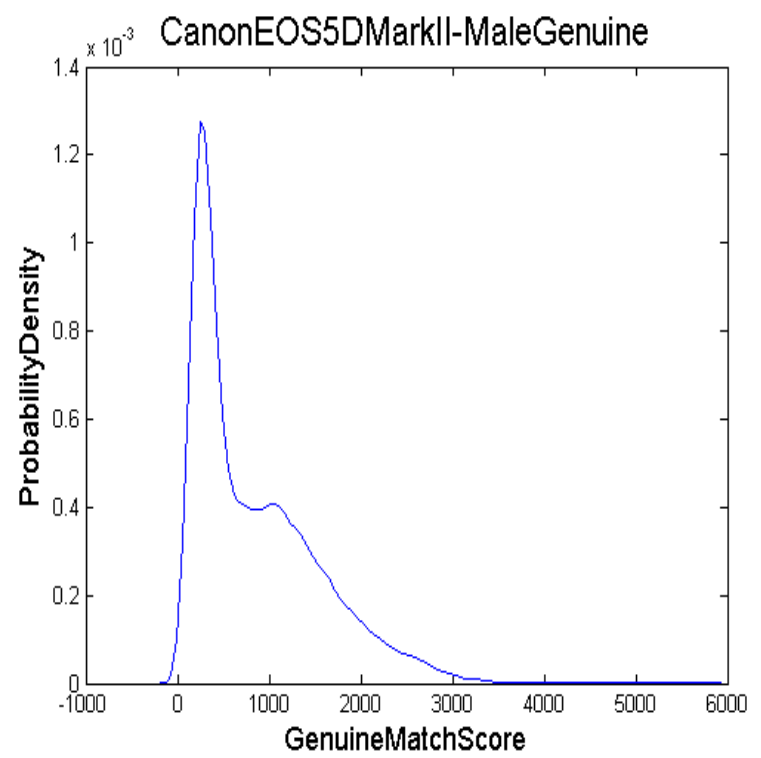

(a)

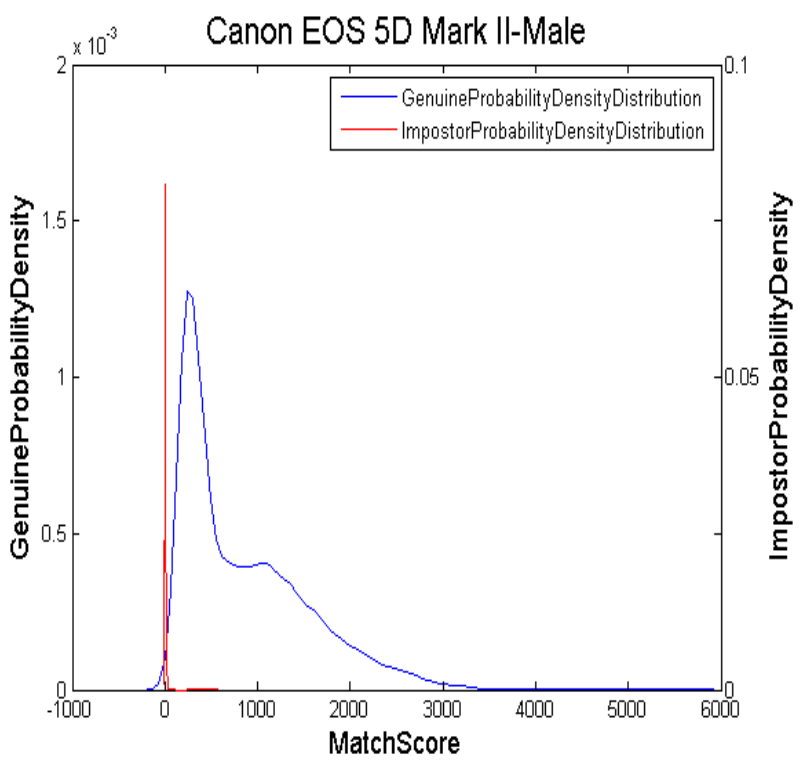

(c)

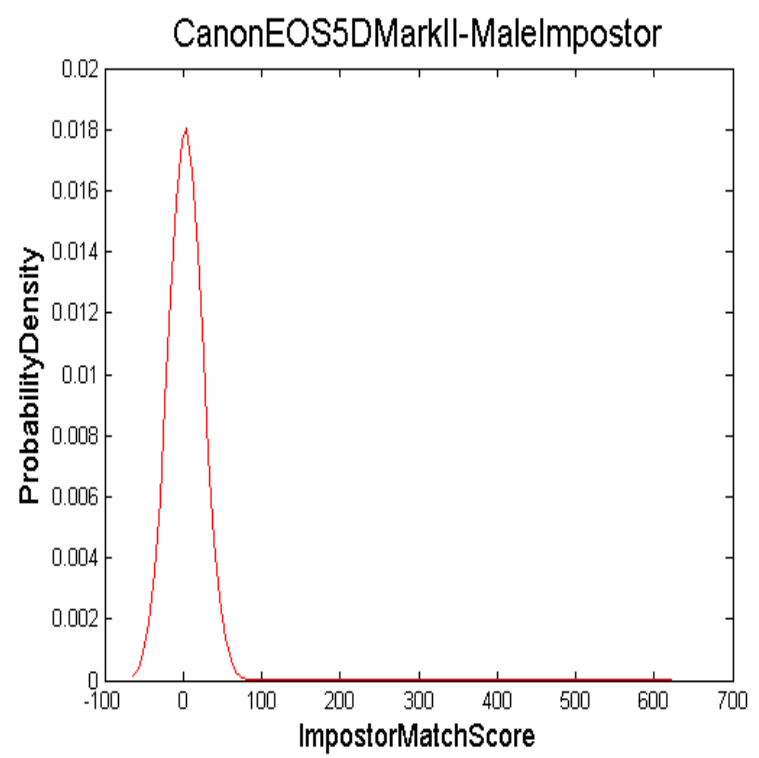

(b)

Figure 4.3: Genuine and imposter score distributions for male face images from Canon EOS 5D Mark II camera.

(a) Genuine distribution of male face. (b) Impostor distribution of male face. (c) Combined distribution of match scores of male face. 


\section{(I) Gender-Based Face ROC Vs Overall Data}

Receiver Operating Characteristics (ROC) of male and female cohorts were plotted against overall data. Figure 4.4 illustrates the performance of each of them. Table 4.3 lists the scores of attributes demonstrating the performance of each of the demographics along with those for complete data.

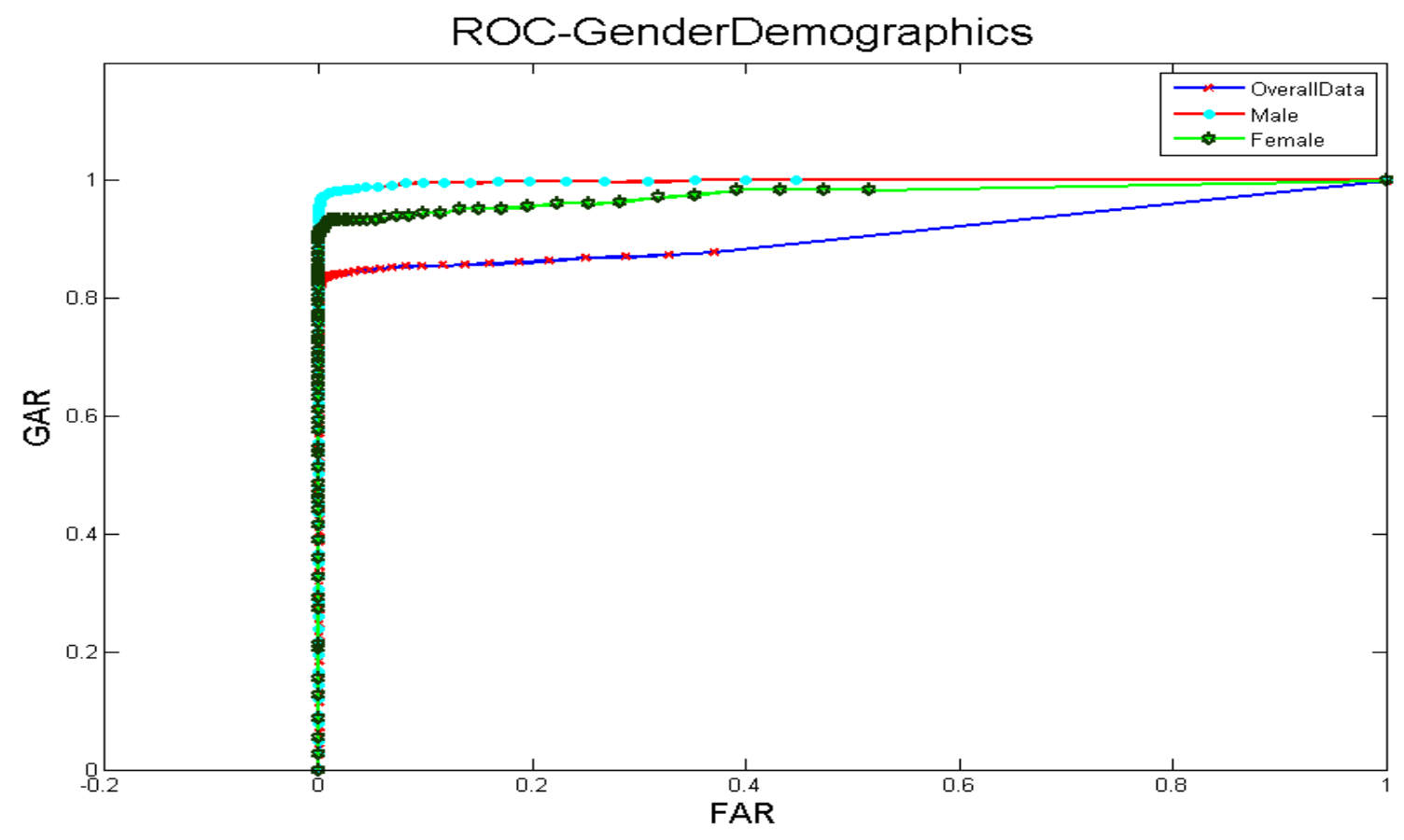

Figure 4.4: ROC-Gender Vs Overall Data

Table 4.3: Scores of attributes related to ROC-Gender Demographics

\begin{tabular}{|c|c|c|}
\hline Demographic/Attribute & Area Under Curve (AUC) & Equal Error Rate (EER) \\
\hline Overall Data & 0.9096 & 0.1367 \\
\hline Female & 0.9750 & 0.0627 \\
\hline Male & 0.9983 & 0.0173 \\
\hline
\end{tabular}

As discussed earlier, it is desirable to have higher area under curve and lower equal error rate for an ROC. See section 2.5 for ideal characteristics of an ROC. For an ideal performance, scores of AUC equals 1 and EER equals 0. Although nearly accurate scores were recorded by all, performance of both genders are relatively accurate than the overall data. AUC and EER of male cohort is very much close to that of an ideal curve. Thus, we can conclude that gender demographics performed slightly better than overall data and male in turn performed better over female counterparts. 


\section{(II) Gender-Based Face Distance Measures}

Kull-back Leibler, Jeffery's and Jensen-Shannon divergence scores were calculated for each gender demographic with respect to total face data. Initially, probability distributions were plotted for entire data set, female and male cohorts as shown in figures 4.1, 4.2 and 4.3. Distance between the distributions of actual data set and each demographic were measured both for genuine and impostor distributions and scores were tabulated in table 4.4.

Table 4.4: Distance scores for Gender Demographics

\begin{tabular}{|c|c|c|c|c|}
\hline \multirow{2}{*}{ Gender/Distribution } & \multicolumn{2}{|c|}{ Genuine } & \multicolumn{2}{c|}{ Impostor } \\
\cline { 2 - 5 } & KLD(or)JD/2 & JSD & KLD(or)JD/2 & JSD \\
\hline Female & 0.2170 & 0.1076 & 0.2986 & 0.1443 \\
\hline Male & 0.1477 & 0.0752 & 0.1575 & 0.0785 \\
\hline
\end{tabular}

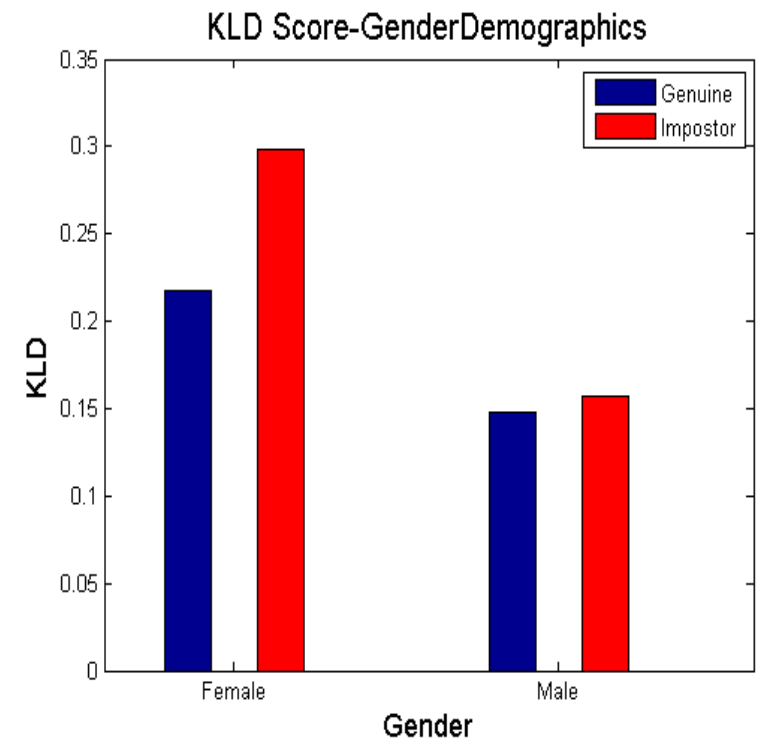

(a)

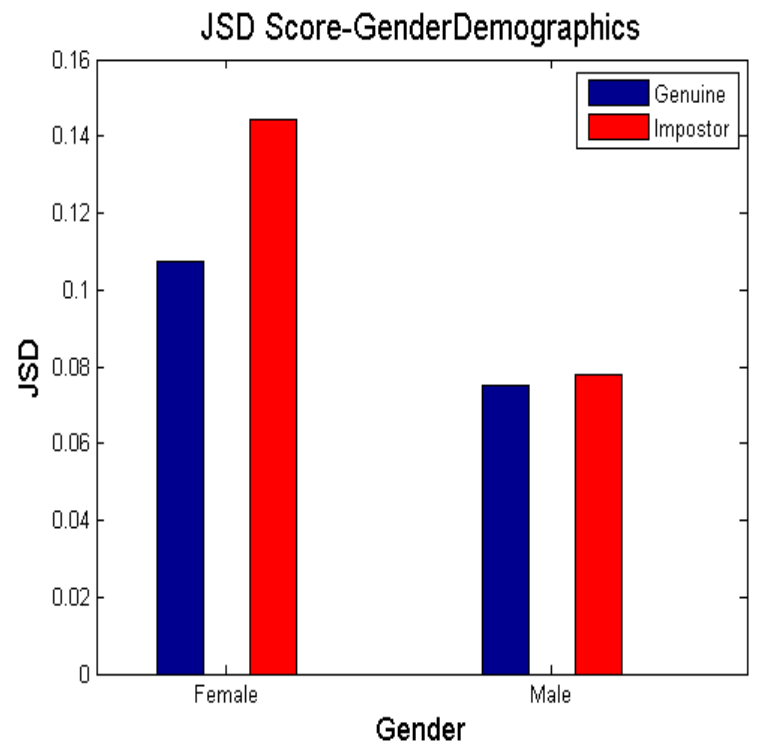

(b)

Figure 4.5: Graph of Distance score-Gender Demographics (a) KLD (b) JSD

Graphical representation of distance scores from above table were shown in figure 4.5. As discussed in section 2.4, it is desirable to have divergence scores close to zero. From the figure above, we can say that distance measures for 'Male' are comparatively better than that of 'Female' in both the genuine and impostor cases. Both the statistical measures exhibited similar trend throughout the gender demographics. 


\subsubsection{Age-Based Face Match Score Distributions}

As discussed earlier, three groups were considered based on the age distribution of participants. For the 31-49, 50-70 age groups, there was no 'out of memory' issue with the matcher, so impostor scores were obtained considering all images in that age range. Due to the large number of participants in the age groups 20-30, a random subset of 1700 was made and impostor scores are extracted using this random subset. Results are summarized in Figures 4.6, 4.7 and 4.8. Table 4.5 gives the brief summary of match scores of all age groups.

Table 4.5: Summary of MatchScores for Age Demographics

\begin{tabular}{|c|c|c|c|c|}
\hline \multirow{2}{*}{$\begin{array}{c}\text { Type of } \\
\text { Demographic }\end{array}$} & Category & \multirow{2}{*}{$\begin{array}{c}\text { Max. } \\
\text { Matchscore }\end{array}$} & \multirow{2}{*}{$\begin{array}{c}\text { Min. } \\
\text { Matchscore }\end{array}$} & \multirow{2}{*}{$\begin{array}{c}\text { Approximate percentage } \\
\text { of maximum } \\
\text { concentration }\end{array}$} \\
\hline & Distribution & & & \\
\hline \multirow{2}{*}{$\begin{array}{l}\text { AgeGroup } \\
20-30\end{array}$} & Genuine & 5268 & 13 & $\begin{array}{c}99 \% \text { of scores ranges } \\
\text { between } 0 \text { and } 3000\end{array}$ \\
\hline & Impostor & 88 & 0 & $\begin{array}{c}99 \% \text { of scores ranges } \\
\text { between } 0 \text { and } 25\end{array}$ \\
\hline \multirow{2}{*}{$\begin{array}{l}\text { AgeGroup } \\
31-49\end{array}$} & Genuine & 4628 & 12 & $\begin{array}{c}99 \% \text { of scores ranges } \\
\text { between } 0 \text { and } 2900\end{array}$ \\
\hline & Impostor & 594 & 0 & $\begin{array}{c}99.9 \% \text { of scores ranges } \\
\text { between } 0 \text { and } 35\end{array}$ \\
\hline \multirow{2}{*}{$\begin{array}{l}\text { AgeGroup } \\
50-70\end{array}$} & Genuine & 5521 & 0 & $\begin{array}{c}99.5 \% \text { of scores ranges } \\
\text { between } 0 \text { and } 3400\end{array}$ \\
\hline & Impostor & 45 & 0 & $\begin{array}{c}95 \% \text { of scores ranges } \\
\text { between } 0 \text { and } 13\end{array}$ \\
\hline
\end{tabular}




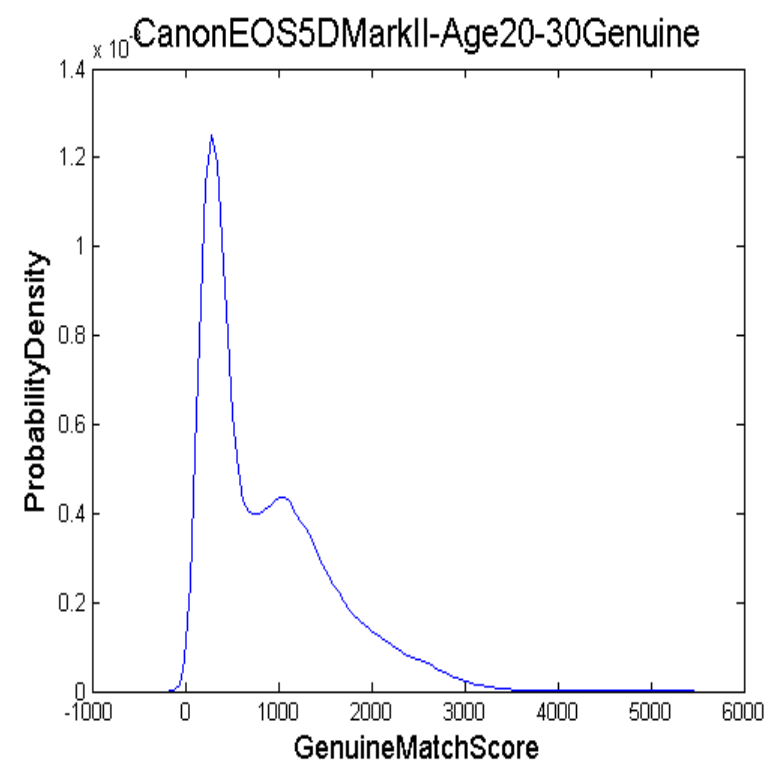

(a)

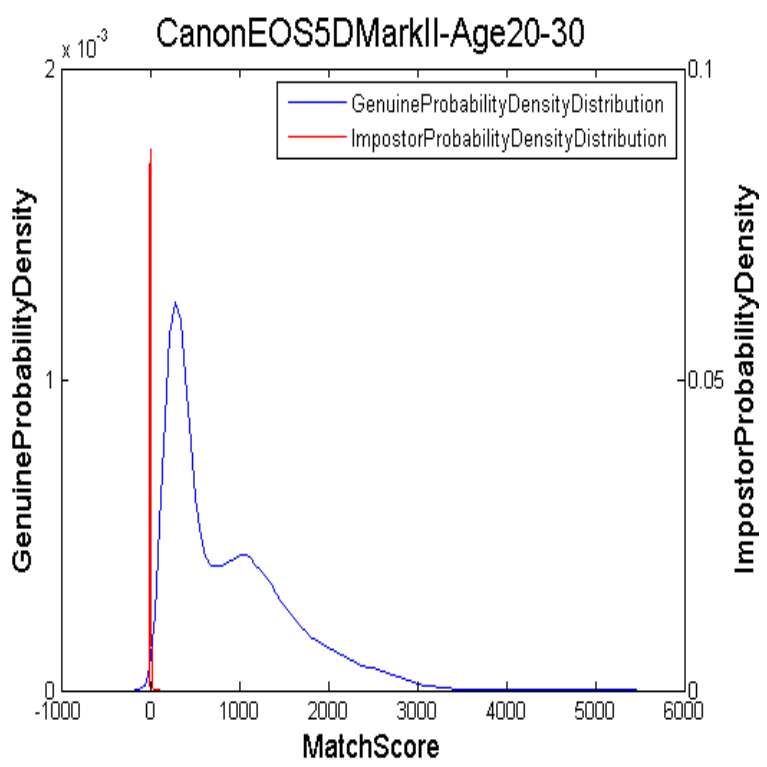

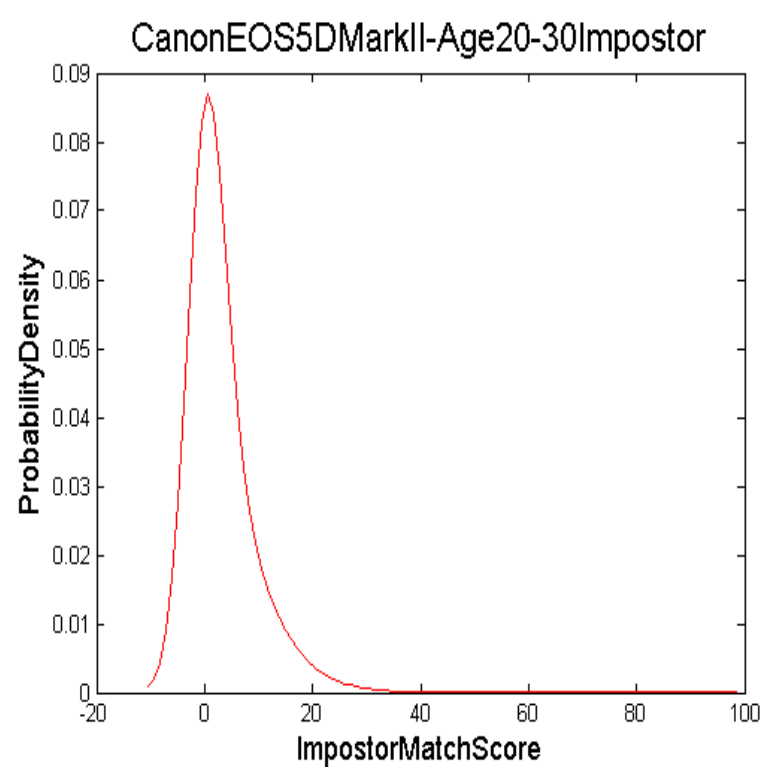

(b)

Figure 4.6: Genuine and imposter score distributions for Age 20-30 face images from Canon EOS 5D Mark II camera.

(a) Genuine distribution of Age 20-30. (b) Impostor distribution of Age 20-30. (c) Combined distribution of match scores of Age 20-30.

(c) 


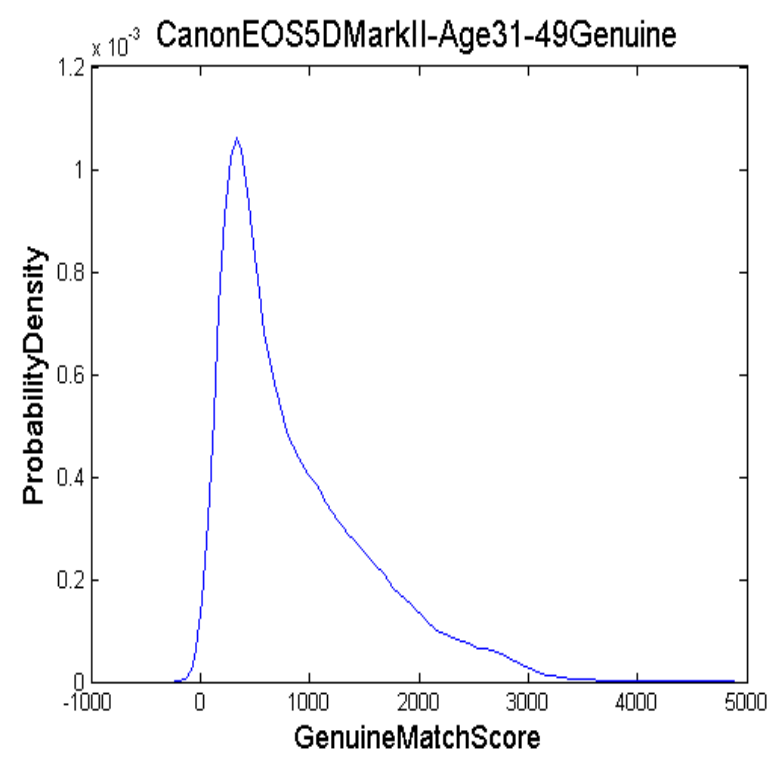

(a)

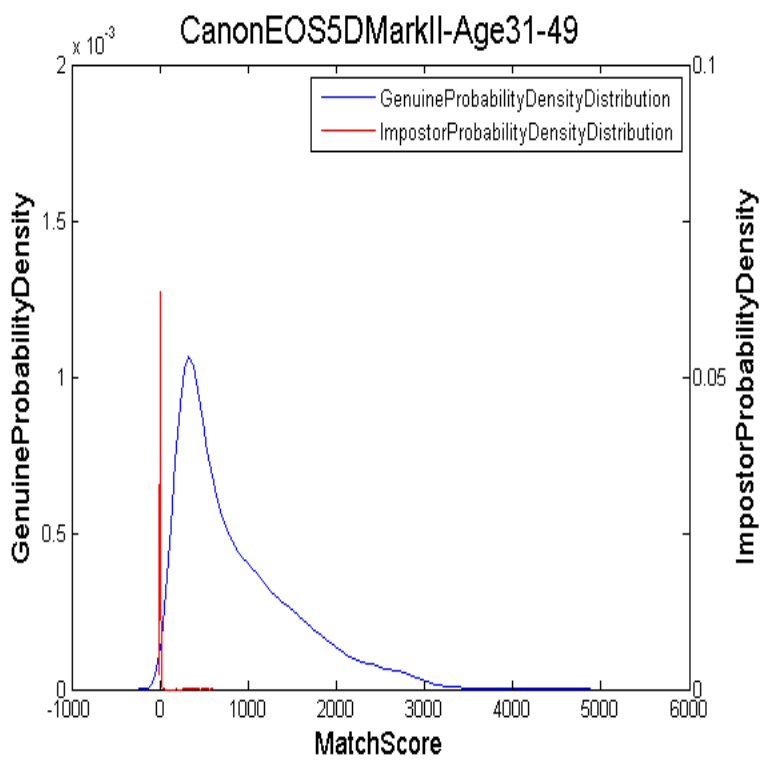

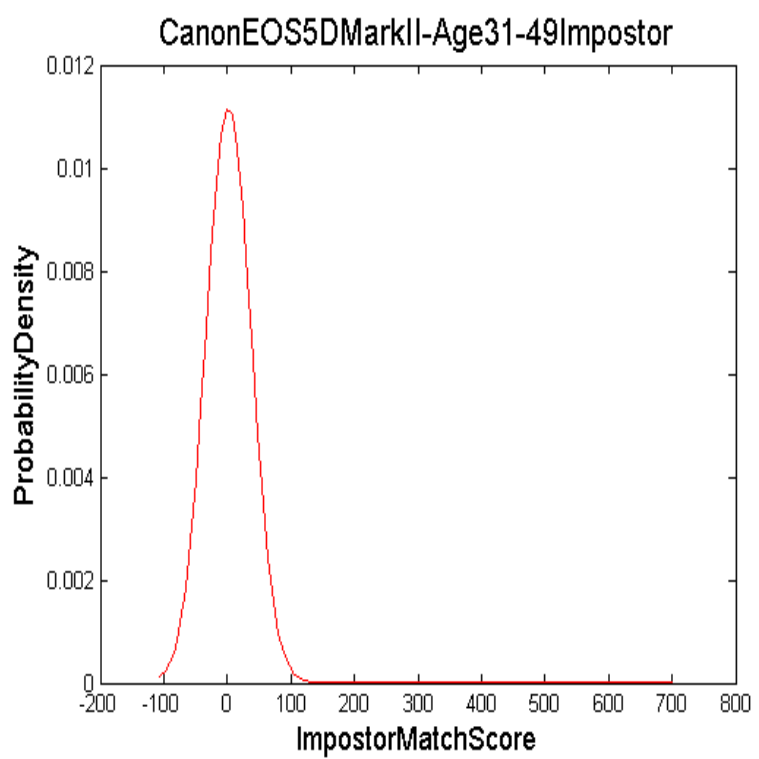

(b)
Figure 4.7: Genuine and imposter score distributions for Age 31-49 face images from Canon EOS 5D Mark II camera.

(a) Genuine distribution of Age 31-49. (b) Impostor distribution of Age 31-49. (c) Combined distribution of match scores of Age 31-49.

(c) 


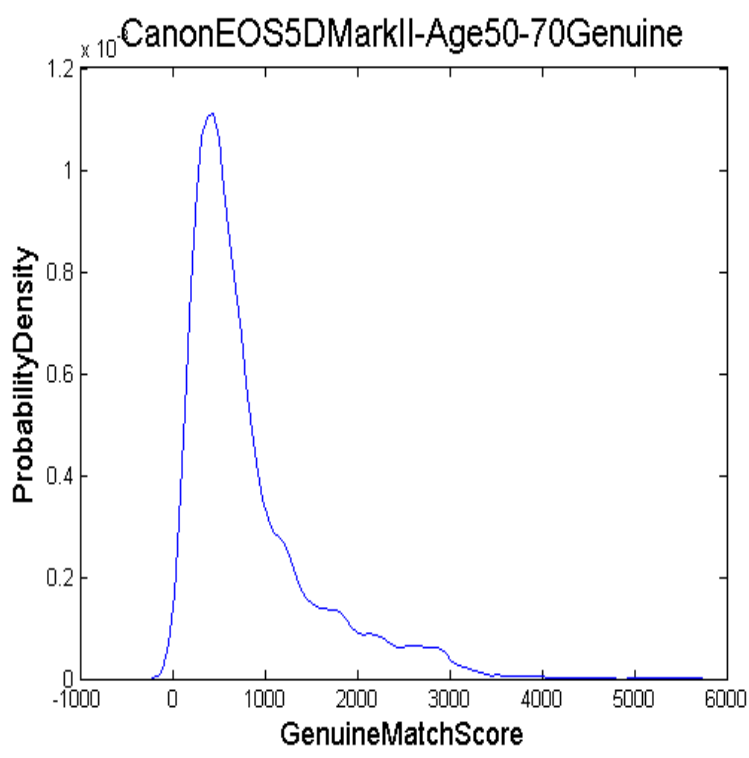

(a)

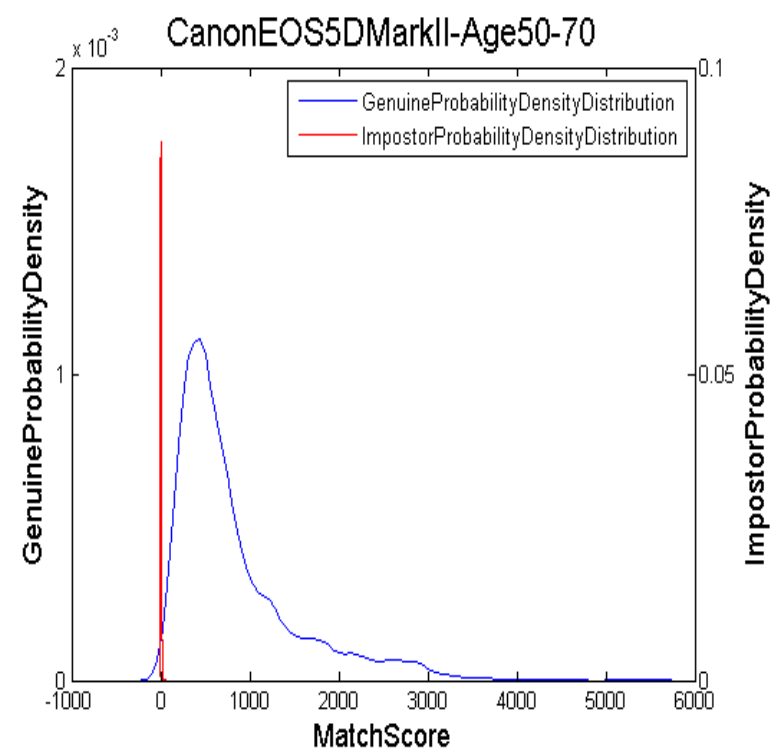

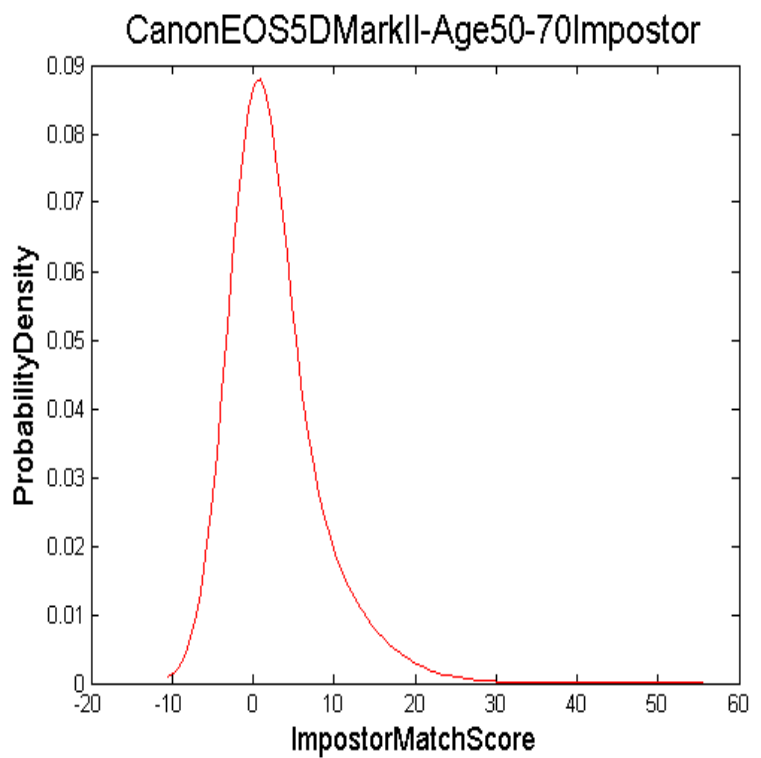

(b)

Figure 4.8: Genuine and imposter score distributions for Age 50-70 face images from Canon EOS 5D Mark II camera.

(a) Genuine distribution of Age 50-70. (b) Impostor distribution of Age 50-70. (c) Combined distribution of match scores of Age 50-70.

(c)

The genuine match score distributions of the 31-49 and 50-70 age groups is noticeable different than both the 20-30 age group and the entire population. This is most likely due to the smaller number of participants/images included in these cohorts. However, there is no significance difference in the overlap region of genuine and imposter score distributions for the age groups 3149 and 50-70 when compared to the total population and age group 20-30. 


\section{(I) Age-Based Face ROC Vs Overall Data}

Figure 4.9 illustrates the ROC of various age groups against overall data. Table 4.6 lists the scores of all attributes for each of these age groups along with those for complete data helpful to evaluate the performance of each group.

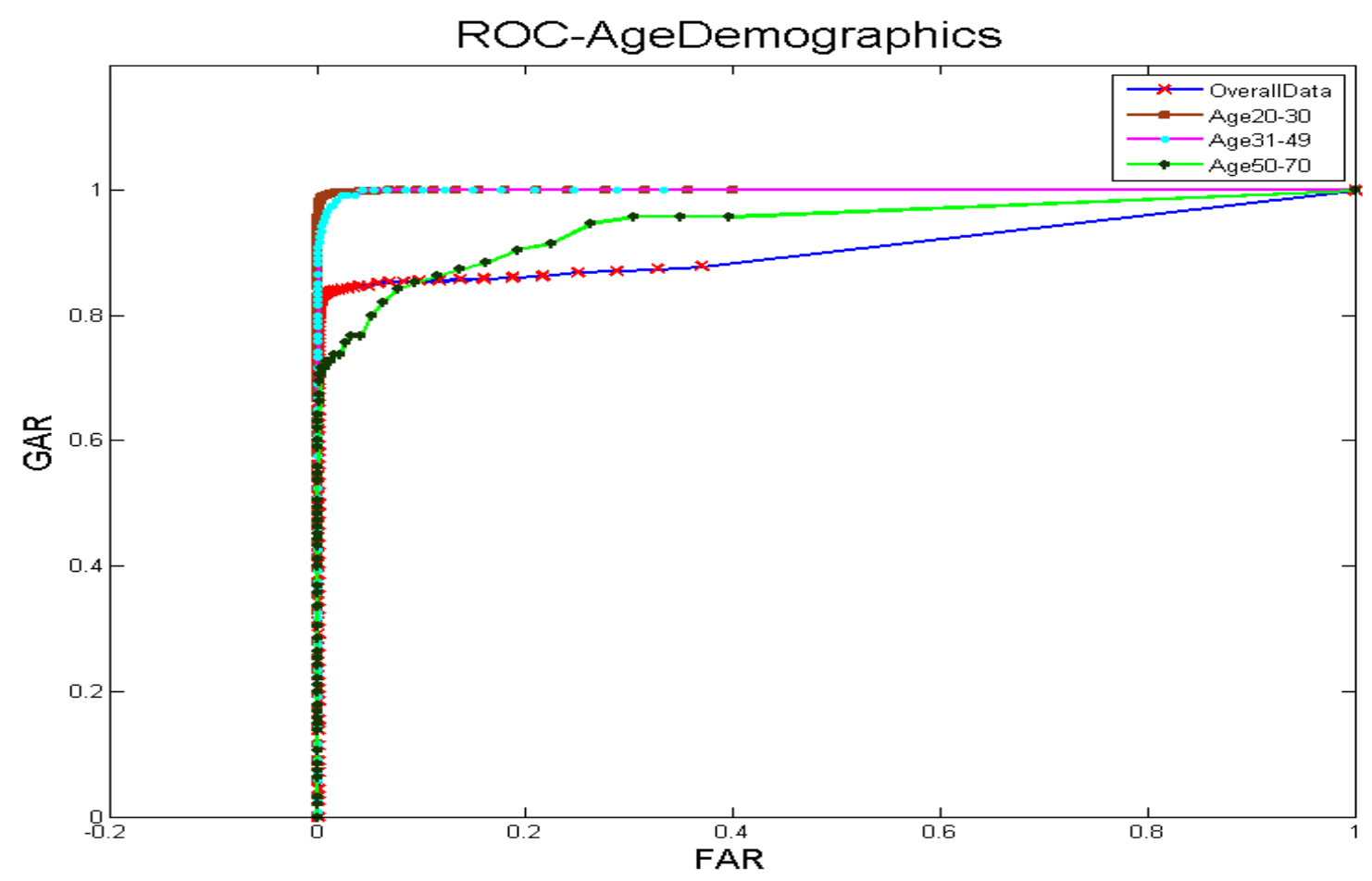

Figure 4.9: ROC Age Groups Vs Overall Data

Table 4.6: Scores of attributes related to ROC-Age Demographics

\begin{tabular}{|c|c|c|}
\hline Demographic/Attribute & Area Under Curve (AUC) & Equal Error Rate (EER) \\
\hline Overall Data & 0.9096 & 0.1367 \\
\hline Age 20-30 & 0.9997 & 0.0095 \\
\hline Age 31-49 & 0.9991 & 0.0180 \\
\hline Age 50-70 & 0.9382 & 0.1290 \\
\hline
\end{tabular}

From the above table, we can say that performance of all the age groups is more or less better than overall data. However, among the age groups less accuracy was shown by people in between 5070 years of age than remaining participants. Age groups 20-30 and 31-49 demonstrated very narrow difference in their performances. Still, age group 20-30 resulted almost high accuracy despite being a cohort with more number of participants. 


\section{(I) Age-Based Face Distance Measure}

Various statistical divergence measures were calculated for each age demographic with respect to total face data. Initially, probability distributions were plotted for entire data set, age 20-30, age 31-49 and age 50-70 cohorts as shown in figures 4.1, 4.6, 4.7 and 4.8. Distance between the distributions of actual data set and each demographic were measured both for genuine and impostor distributions and scores were tabulated in table 4.7.

Table 4.7: Distance scores for Age Demographics

\begin{tabular}{|c|c|c|c|c|}
\hline \multirow{2}{*}{ Age/Distribution } & \multicolumn{2}{|c|}{ Genuine } & \multicolumn{2}{c|}{ Impostor } \\
\cline { 2 - 5 } & KLD(or)JD/2 & JSD & KLD(or)JD/2 & JSD \\
\hline Age 20-30 & 0.0877 & 0.0450 & 0.0609 & 0.0304 \\
\hline Age 31-49 & 0.3899 & 0.2090 & 0.0819 & 0.0415 \\
\hline Age 50-70 & 0.5064 & 0.2945 & 0.0679 & 0.0338 \\
\hline
\end{tabular}

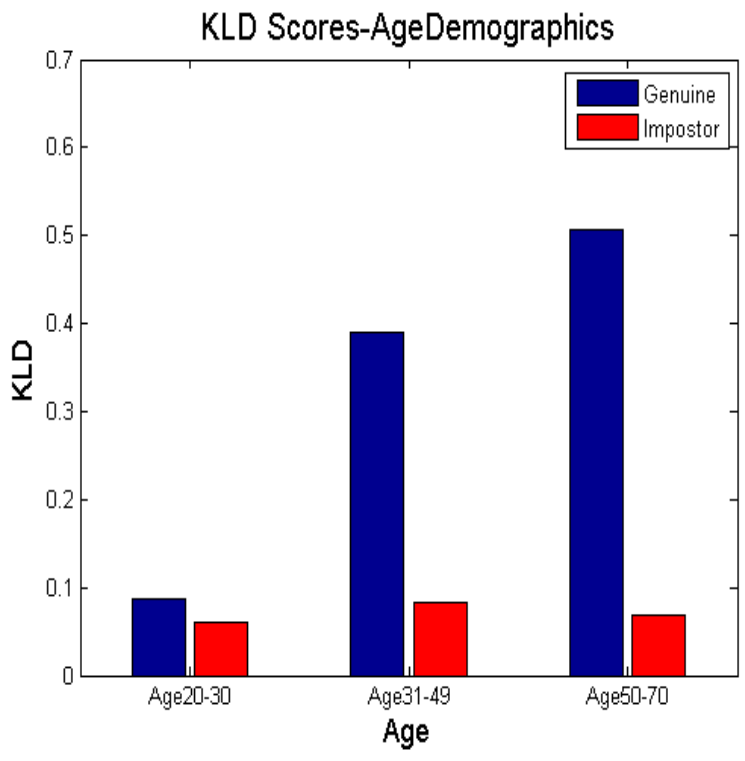

(a)

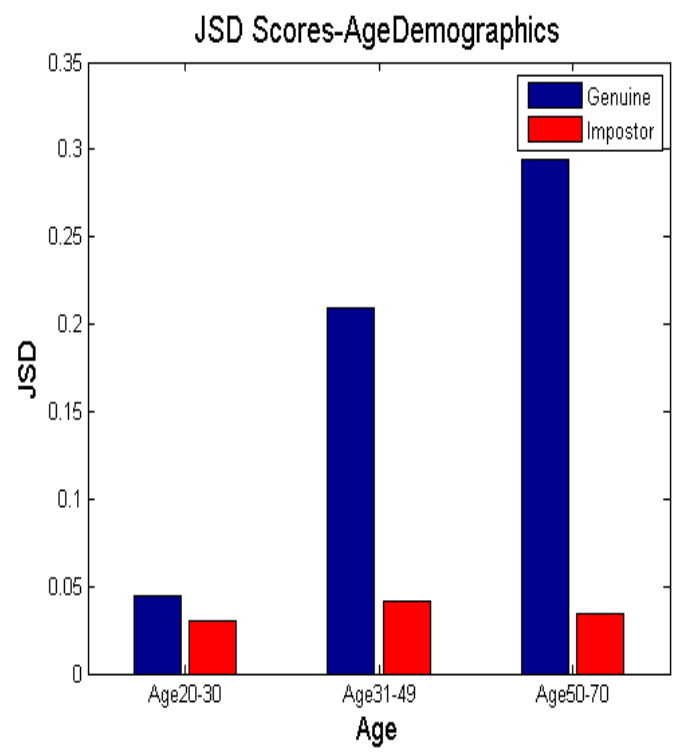

(b)

Figure 4.10: Graph of Distance score-Age Demographics (a) KLD (b) JSD

Graphical representation of distance scores from above table were shown in figure 4.10. From the graph above, we can say that distance measures for Age group 20-30 is drastically less than that of ages 31-49 and 50-70 in genuine case which is desirable. This is perhaps due to large sample size of this cohort. In impostor case, not much difference is observed among the scores, however, they are very much close to ' 0 '. Similar trend was observed in both KLD and JSD. 


\subsubsection{Ethinicity-Based Face Match Score Distributions}

Face images were grouped according to the ethnicities described in section 3.1. For imposter score generation, all images for each ethnicity were considered in the matching galleries except for Caucasians due the "out of memory" issue experienced due to the number of Caucasian images. In this case, a random subset of 1700 gallery images was created on which impostor matching was performed for each Caucasian probe image. A comparison of the aggregate face combined genuine and imposter score distributions and the individual combined distributions for each ethnicity are provided in Figures 4.11-1 and 4.11-2. Table 4.8 lists the set of match scores that are significant to each ethnicity. A comprehensive listing of individual ethnicity-based genuine and imposter face image score distributions is provided in Appendix A.

Overall, as observed in Figures 4.11-1 \& 4.11-2, there is no major change in the overlap region of the imposter and genuine scores for any ethnicity when compared to the aggregate scores (Figure 4.11(a)); again when considering the relative scales of the probability density values on the $y$ axes). Any changes in distributions can be attributed to the variation is the sizes of the ethnic populations within the dataset. 
Table 4.8: Summary of MatchScores for Ethnicity Demographics

\begin{tabular}{|c|c|c|c|c|}
\hline \multirow{2}{*}{$\begin{array}{c}\text { Type of } \\
\text { Demographic }\end{array}$} & Category & \multirow{2}{*}{$\begin{array}{l}\text { Max. } \\
\text { Match } \\
\text { score }\end{array}$} & \multirow{2}{*}{$\begin{array}{l}\text { Min. } \\
\text { Match } \\
\text { score }\end{array}$} & \multirow[b]{2}{*}{$\begin{array}{c}\text { Approximate percentage of maximum } \\
\text { concentration }\end{array}$} \\
\hline & Distribution & & & \\
\hline \multirow[t]{2}{*}{ African } & Genuine & 3201 & 34 & $\begin{array}{l}99.5 \% \text { of scores ranges between } 0 \text { and } \\
\qquad 2500\end{array}$ \\
\hline & Impostor & 44 & 0 & $97 \%$ of scores ranges between 0 and 30 \\
\hline \multirow{2}{*}{$\begin{array}{l}\text { African- } \\
\text { American }\end{array}$} & Genuine & 3470 & 42 & $\begin{array}{l}99 \% \text { of scores ranges between } 0 \text { and } \\
2700\end{array}$ \\
\hline & Impostor & 321 & 0 & $\begin{array}{l}99.9 \% \text { of scores ranges between } 0 \text { and } \\
45\end{array}$ \\
\hline \multirow[t]{2}{*}{ Asian } & Genuine & 4696 & 16 & $\begin{array}{l}99 \% \text { of scores ranges between } 0 \text { and } \\
\qquad 2800\end{array}$ \\
\hline & Impostor & 88 & 0 & $99 \%$ of scores ranges between 0 and 38 \\
\hline \multirow[t]{2}{*}{ Asian-Indian } & Genuine & 4637 & 43 & $\begin{array}{l}99 \% \text { of scores ranges between } 0 \text { and } \\
2800\end{array}$ \\
\hline & Impostor & 88 & 0 & $99 \%$ of scores ranges between 0 and 35 \\
\hline \multirow{2}{*}{ Caucasian } & Genuine & 5730 & 0 & $\begin{array}{l}99 \% \text { of scores ranges between } 0 \text { and } \\
3200\end{array}$ \\
\hline & Impostor & 558 & 0 & $\begin{array}{l}99.5 \% \text { of scores ranges between } 0 \text { and } \\
30\end{array}$ \\
\hline \multirow[t]{2}{*}{ Hispanic } & Genuine & 4560 & 46 & $\begin{array}{l}99.5 \% \text { of scores ranges between } 0 \text { and } \\
3200\end{array}$ \\
\hline & Impostor & 63 & 0 & $95 \%$ of scores ranges between 0 and 17 \\
\hline \multirow{2}{*}{$\begin{array}{l}\text { Middle- } \\
\text { Eastern }\end{array}$} & Genuine & 5052 & 13 & $\begin{array}{l}99.5 \% \text { of scores ranges between } 0 \text { and } \\
3000\end{array}$ \\
\hline & Impostor & 58 & 0 & $99 \%$ of scores ranges between 0 and 27 \\
\hline \multirow{2}{*}{$\begin{array}{l}\text { Pacific } \\
\text { Islanders }\end{array}$} & Genuine & 2709 & 67 & $\begin{array}{l}99.5 \% \text { of scores ranges between } 0 \text { and } \\
\qquad 2400\end{array}$ \\
\hline & Impostor & 27 & 0 & $\begin{array}{l}99.5 \% \text { of scores ranges between } 0 \text { and } \\
20\end{array}$ \\
\hline \multirow[t]{2}{*}{ Others } & Genuine & 3518 & 56 & $\begin{array}{l}95 \% \text { of scores ranges between } 0 \text { and } \\
2300\end{array}$ \\
\hline & Impostor & 36 & 0 & $95 \%$ of scores ranges between 0 and 15 \\
\hline
\end{tabular}




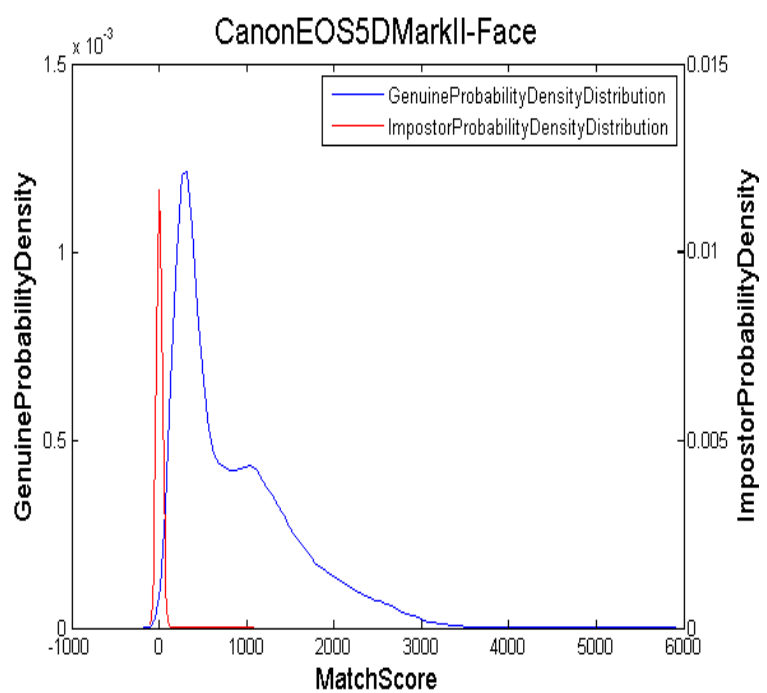

(a)

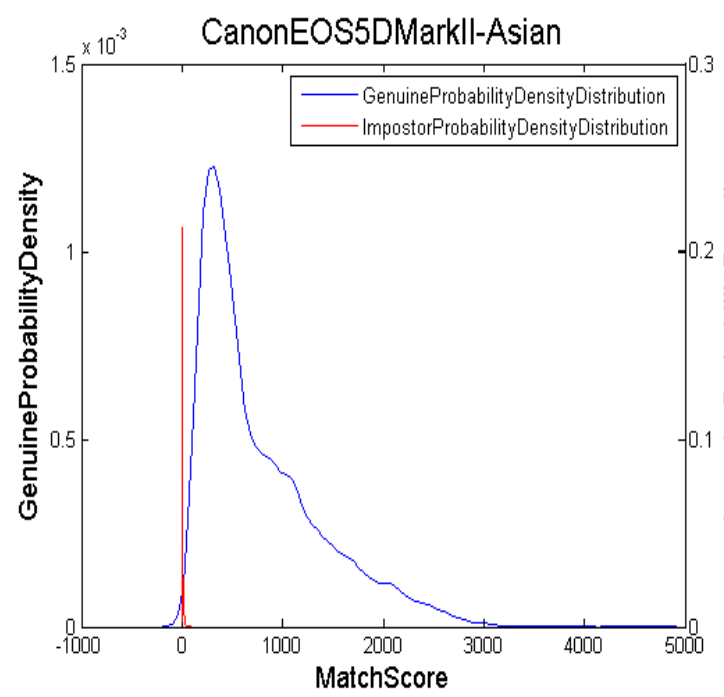

(c)

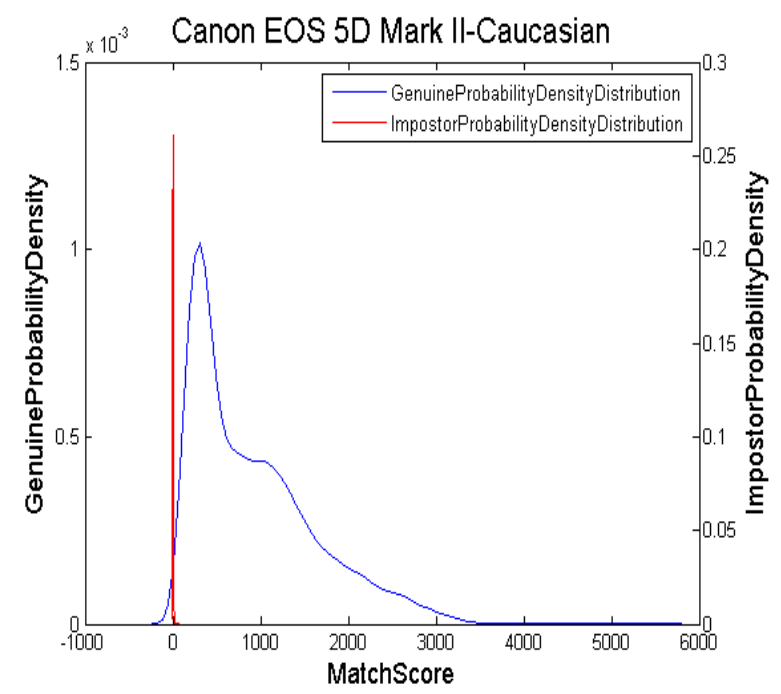

(e)

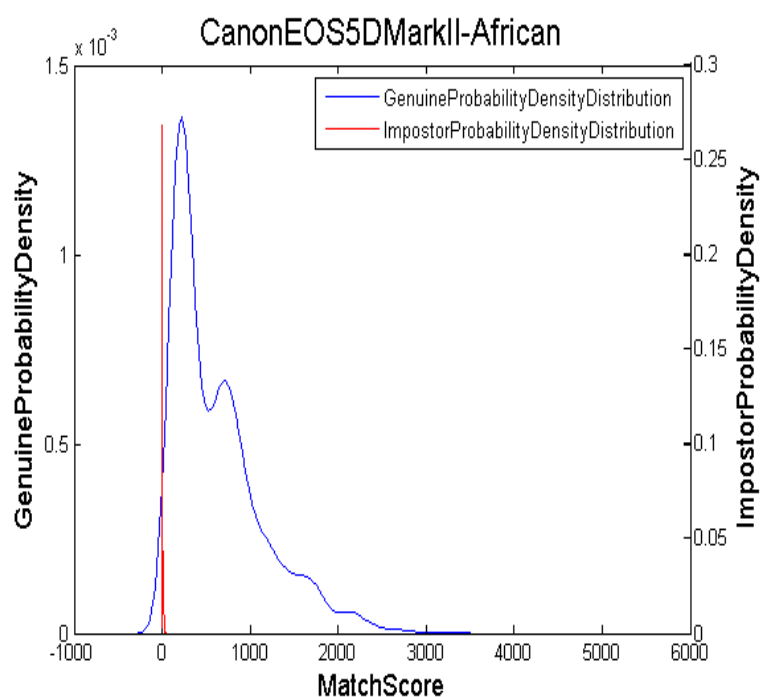

(b)

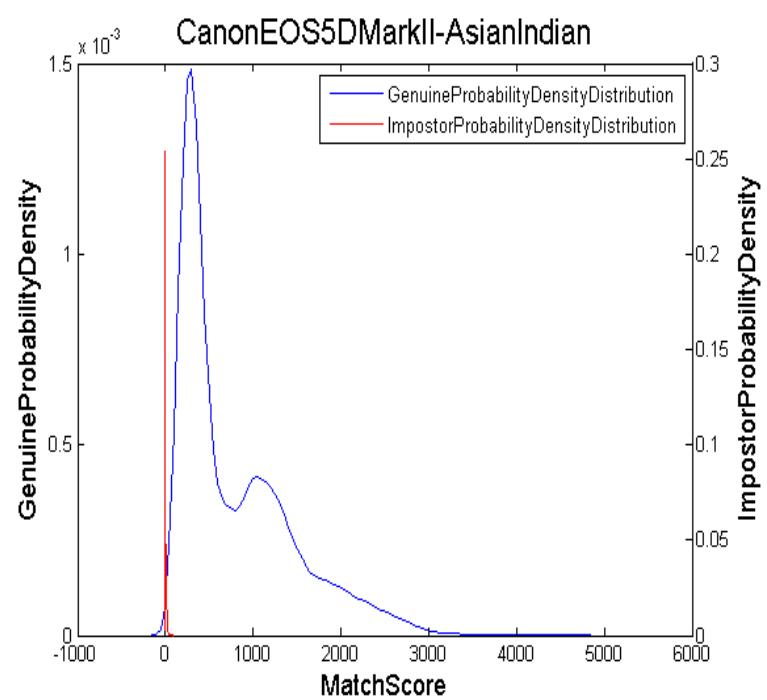

(d)

Figure 4.11-1: Combined match score distributions for ethnicities from Canon EOS 5D Mark II camera.

(a) Face (aggregate) (b) African (c) Asian (d) AsianIndian (e) Caucasian 


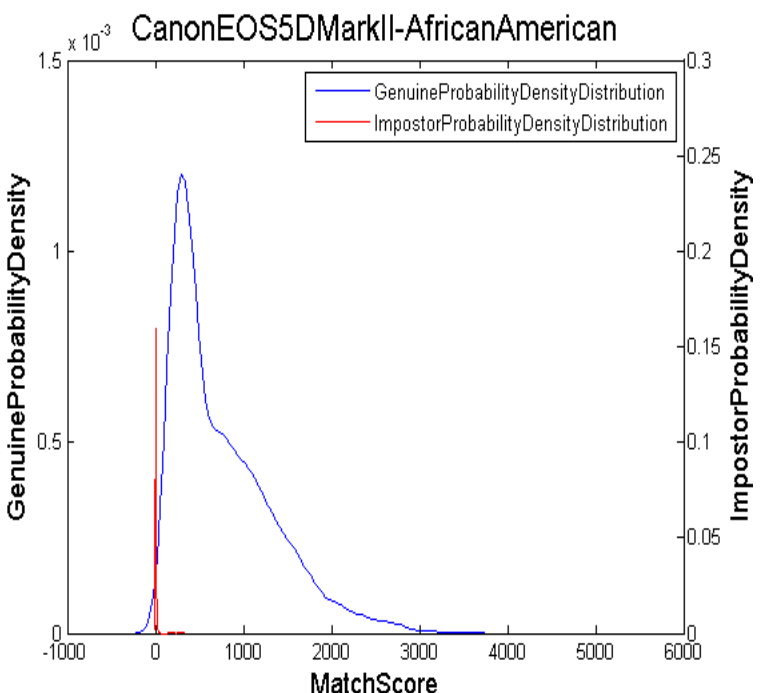

(f)

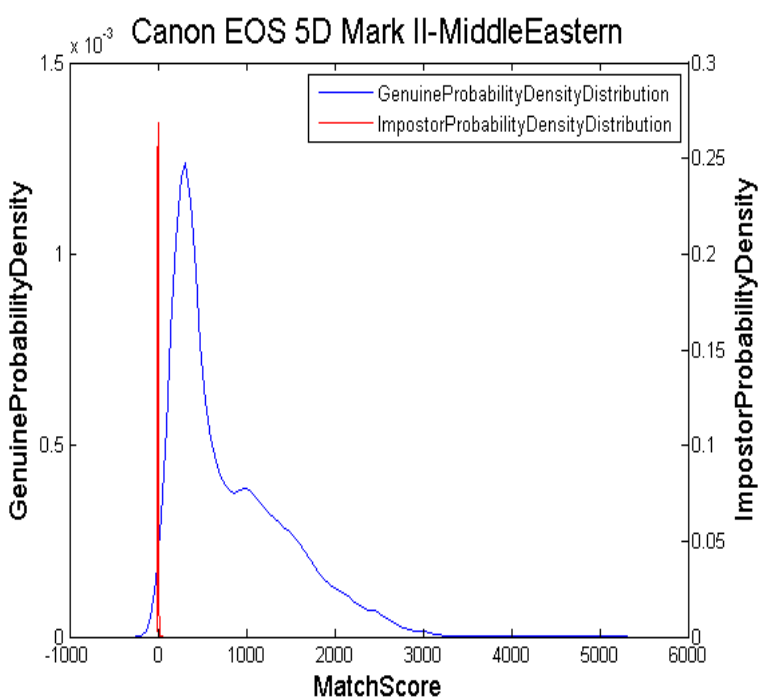

(h)

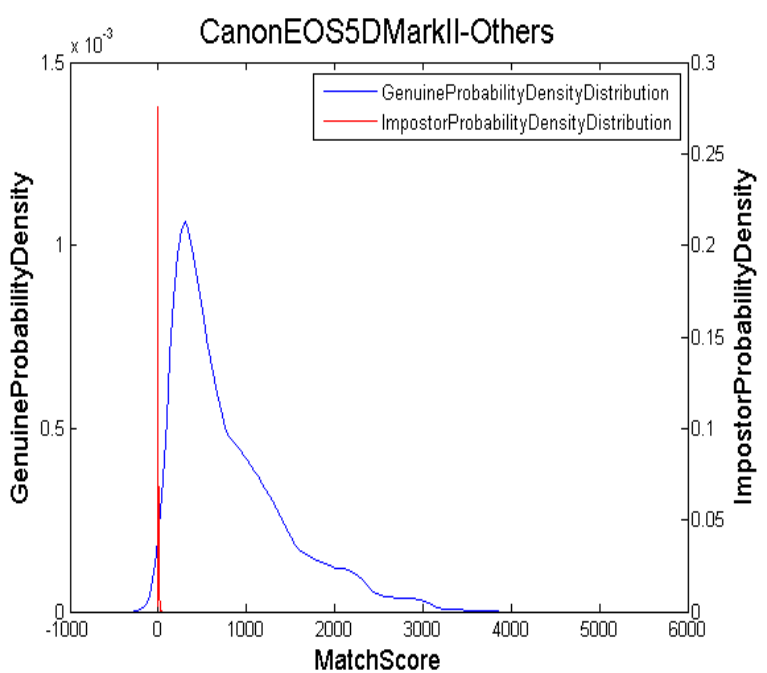

(j)

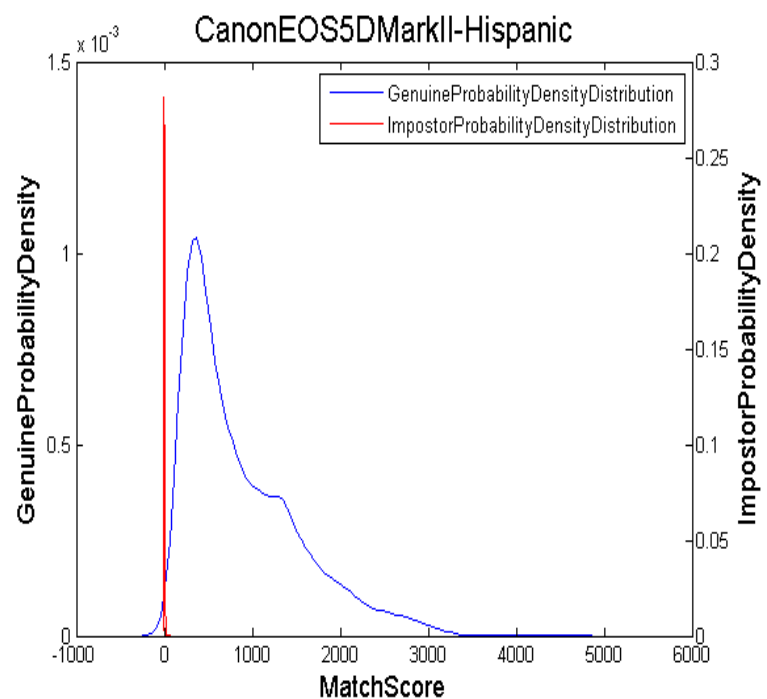

(g)

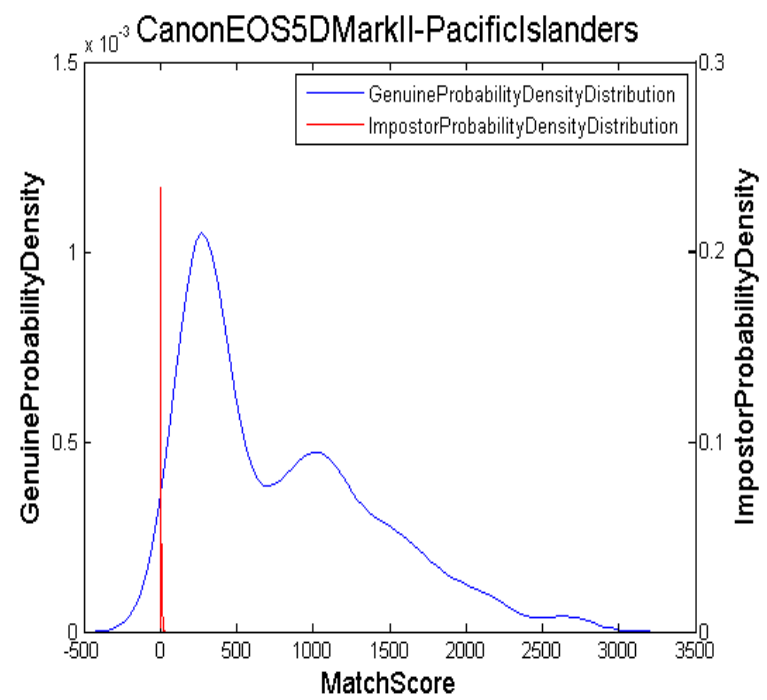

(i)

Figure 4.11-2: Combined match score distributions for ethnicities from Canon EOS 5D Mark II camera.

(f)African-American (g) Hispanic (h) Middle-Eastern (i) Pacific-Islanders (j) Others. 


\section{(I) Ethnicity-Based Face ROC Vs Overall Data}

ROC of all ethnicities along with overall data can be seen in figure 4.12. Table 4.9 lists the scores for all attributes essential to evaluate the performance of each of these ethnicities along with those for overall data.

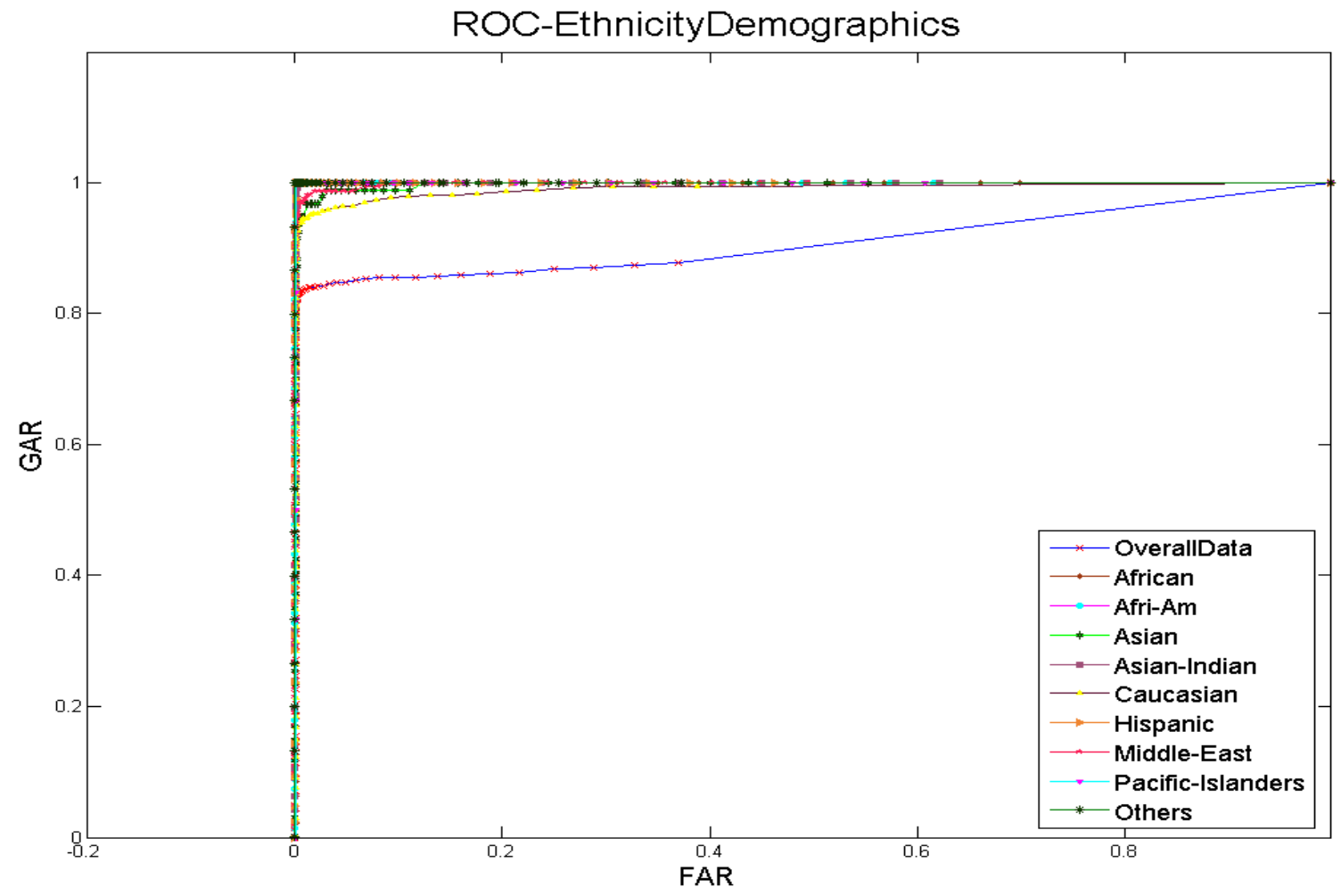

Figure 4.12: ROC-Ethnicities Vs Overall Data

Table 4.9: Scores of attributes related to ROC-Ethnicity Demographics

\begin{tabular}{|c|c|c|}
\hline Demographic/Attribute & Area Under Curve (AUC) & Equal Error Rate (EER) \\
\hline Overall Data & 0.9096 & 0.1367 \\
\hline African & 0.9999 & 0.0020 \\
\hline African-American & 1 & $\sim 0$ \\
\hline Asian & 0.9977 & 0.024 \\
\hline Asian-Indian & 1 & $\sim 0$ \\
\hline Caucasian & 0.9903 & 0.0391 \\
\hline Hispanic & 1 & $\sim 0$ \\
\hline Middle-Eastern & 0.9987 & 0.0160 \\
\hline Pacific-Islanders & 0.9999 & 0.0020 \\
\hline Others & 1 & $\sim 0$ \\
\hline
\end{tabular}


Similar to gender and age demographics, all ethnicities showed better performance over complete data. Among the ethnicities, $100 \%$ accuracy was shown by African-Americans, Asian-Indians, Hispanic and Others. Also, almost ideal performance was observed in Africans, Middle-Eastern and Pacific-Islanders. Caucasians being a largest ethnic group, showed high accuracy over complete data and slightly less than other ethnicities.

\section{(I) Ethnicity-Based Face Distance Measure}

$\mathrm{KL}, \mathrm{J}$ and JS divergence scores were calculated for each ethnic groups with respect to total face data. Initially, probability distributions were plotted for entire data set and all ethnicity cohorts as shown in figures 4.11-1 and 4.11-2. Distance between the distributions of actual data set and each demographic were measured for both genuine and impostor distributions and scores were tabulated in table 4.10 .

Table 4.10: Distance scores for Ethnicity Demographic

\begin{tabular}{|c|c|c|c|c|}
\hline \multirow{2}{*}{ Ethnicity/Distribution } & \multicolumn{2}{|c|}{ Genuine } & \multicolumn{2}{c|}{ Impostor } \\
\cline { 2 - 5 } & KLD(or)JD/2 & JSD & KLD(or)JD/2 & JSD \\
\hline African & 0.7213 & 0.4935 & 0.7113 & 0.3230 \\
\hline African-American & 0.4637 & 0.2890 & 0.5183 & 0.2412 \\
\hline Asian & 0.3591 & 0.2032 & 0.3969 & 0.1834 \\
\hline Asian-Indian & 0.3290 & 0.1750 & 0.5313 & 0.2462 \\
\hline Caucasian & 0.1392 & 0.0680 & 0.0363 & 0.0181 \\
\hline Hispanic & 0.4642 & 0.2790 & 0.1892 & 0.0933 \\
\hline Middle-Eastern & 0.4238 & 0.2494 & 0.2510 & 0.1240 \\
\hline Pacific-Islanders & 1.1263 & 0.6473 & 0.5005 & 0.2438 \\
\hline Others & 0.6318 & 0.4248 & 0.0240 & 0.0122 \\
\hline
\end{tabular}

Graphical representation of distance scores from above table were shown in figure 4.13. From the graph below, we can say that distance measures for ethnicities 'Caucasian' is comparatively less than remaining ethnicities while it is significantly higher for 'Pacific-Islanders' because of its smaller size. Also, distance measure is seen considerably higher in 'Africans' and 'Others' ethnic groups because of their respective sample sizes. 


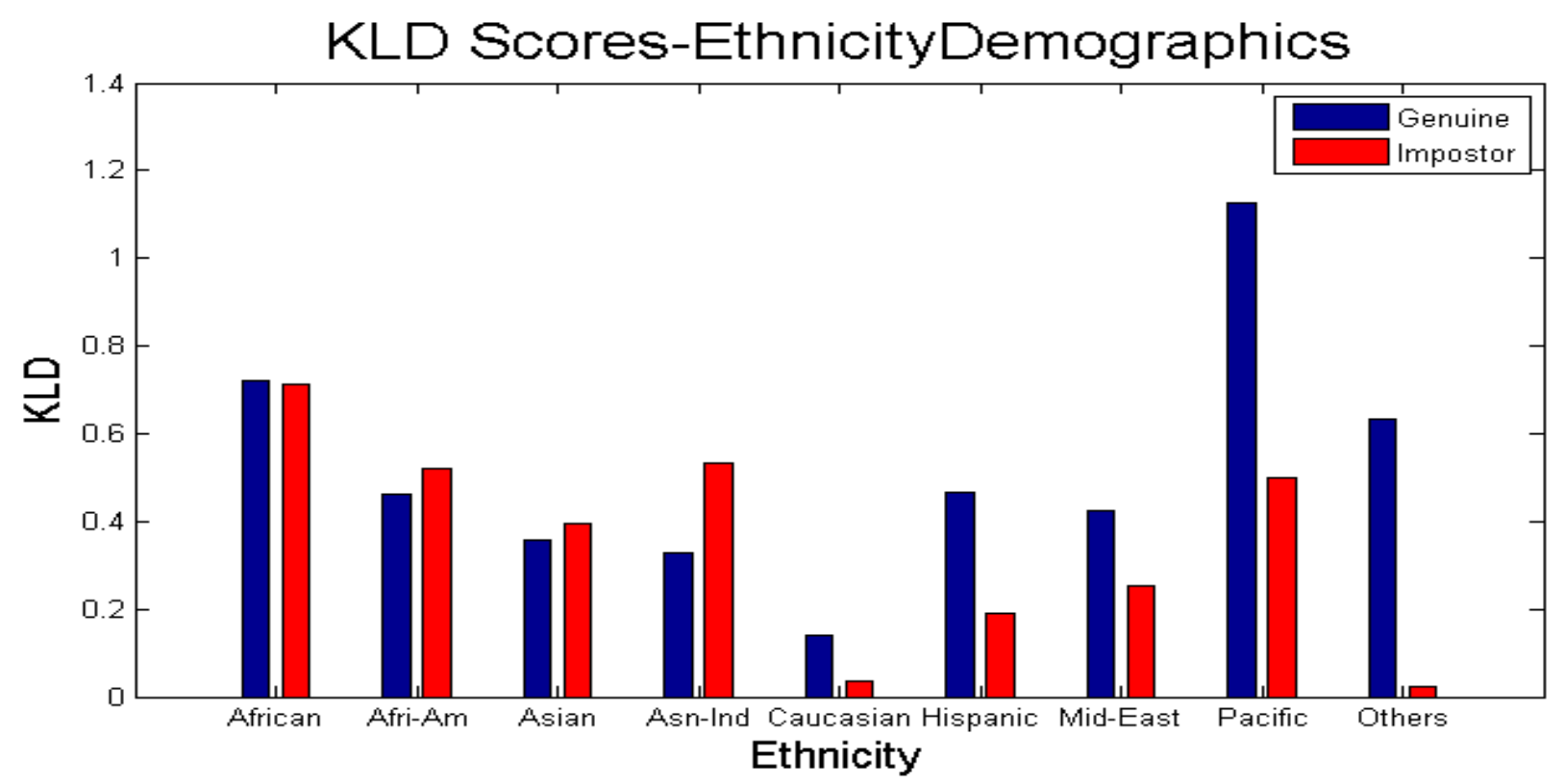

(a)

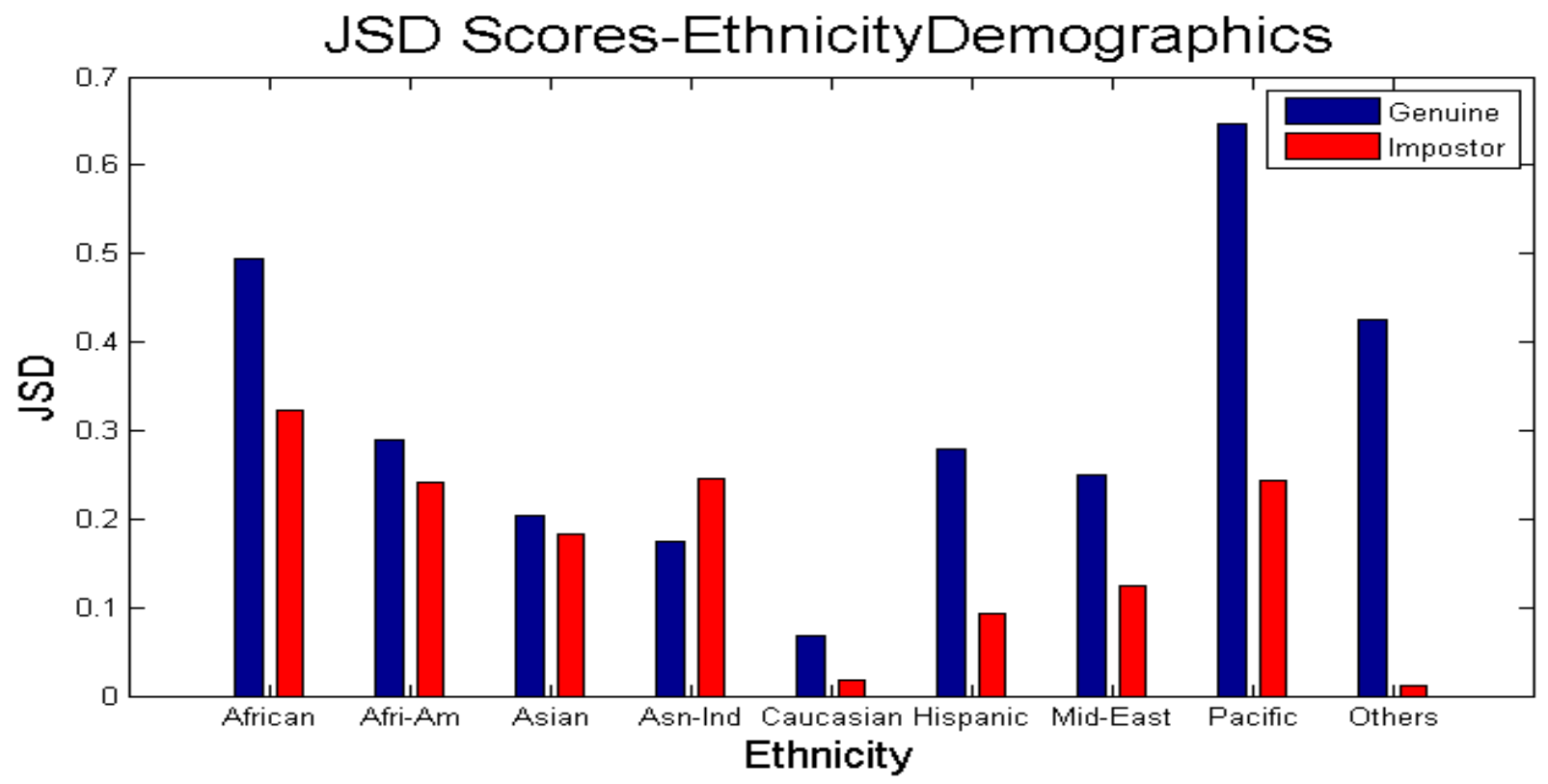

(b)

Figure 4.13: Graph of Distance score-Ethnicity Demographics (a) KLD (b) JSD

In impostor case, there is a large variation of distance scores are ranging from 0.02 for 'Others' to $\sim 0.7$ for 'Africans' in KL and 0.32 to 0.01 in JS divergences. This is perhaps due to the occurrence of more false matches while performing impostor matching on 'Africans', 'African-Americans', Asian-Indians' and 'Pacific-Islanders'. Also, we can say that 'Caucasians' has got minimal 
distance scores in both genuine and impostor cases. Lesser divergence in impostor case implies occurrence of fewer number of false matches.

\section{Statistical Distance Measures among the demographics}

Kullback-Leibler and Jensen-Shannon divergence measures were calculated between cohorts of almost equal sample size. Table 4.11 lists the distance measures observed through pairwise comparison.

Table 4.11: Pairwise Comparison of Demographics MatchScores

\begin{tabular}{|c|c|c|c|}
\hline \multirow{2}{*}{ Demographic Pairs } & Type of Match Scores & KLD (or) JD/2 & JSD \\
\hline Female--Male & Genuine & 0.3887 & 0.1917 \\
\cline { 2 - 4 } & Impostor & 0.1793 & 0.0860 \\
\hline Age20-30--Age31-49 & Genuine & 0.3583 & 0.1931 \\
\hline Age20-30--Age50-70 & Genuine & 0.4384 & 0.2563 \\
\hline Asian-Indian--Asian & Genuine & 0.4805 & 0.2622 \\
\hline Middle-East--Hispanic & Genuine & 0.5091 & 0.3469 \\
\hline $\begin{array}{c}\text { African-America-- } \\
\text { Middle-Eastern }\end{array}$ & Genuine & 0.5013 & 0.3442 \\
\hline $\begin{array}{c}\text { African-America-- } \\
\text { Hispanic }\end{array}$ & Genuine & 0.4839 & 0.3522 \\
\hline African--Others & Genuine & 0.4763 & 0.2988 \\
\hline
\end{tabular}

The observed distance scores did not follow any particular trend although the sample sizes of cohorts are same. Thus, it can be said that sample size of a cohort is not impacting the distance measures in case of demographic pairs.

\section{Error Rates observed in various Demographics}

FRR was calculated for all generated Matchscore distributions at FAR $=1 \%$ and tabulated in Table 4.12. Except for age group 50-70, false reject rate decreased in all demographics than in overall data. But the matcher may yield different error rates at different percentage of FAR. Increase in threshold results in decreased FARs and increased FRRs as there are inversely related. Also, significant increase in failure to enroll rate (FER) was found in Africans and African- Americans. 
Thus, we can say that this commercial matcher failed in creating templates for more number of participants from these ethnicities.

Table 4.12: Percentage of Error Rates Observed in the Dataset

\begin{tabular}{|c|c|c|}
\hline $\begin{array}{c}\text { Matchscore } \\
\text { Distribution }\end{array}$ & $\begin{array}{c}\text { Percentage of False } \\
\text { Rejection (FRR) at } \\
\text { FAR=1\% }\end{array}$ & $\begin{array}{c}\text { Percentage of } \\
\text { Failure to Enroll } \\
(\text { FTE) }\end{array}$ \\
\hline Overall Data & 0.27 & 1.15 \\
\hline Female & 0.05 & 0.7 \\
\hline Male & 0.04 & 2 \\
\hline Age 20-30 & 0.01 & 1.2 \\
\hline Age 31-49 & 0.035 & 2.1 \\
\hline Age 50-70 & 0.60 & 4.5 \\
\hline African & 0 & 19.8 \\
\hline African-American & 0 & 11.9 \\
\hline Asian & 0.06 & 0.7 \\
\hline Asian-Indian & 0 & 1.95 \\
\hline Caucasian & 0.07 & 0.2 \\
\hline Hispanic & 0 & 0.2 \\
\hline Middle-Eastern & 0.08 & 0 \\
\hline Pacific-Islanders & 0 & 0 \\
\hline Others & 0 & 0 \\
\hline
\end{tabular}




\section{Chapter 5}

\section{Conclusion and Future Work}

This chapter summarizes our work and establishes a discussion of contribution, advantages and limitations of our approach, suggesting some direction for future research in the later section.

\subsection{Conclusion}

A commercial matcher namely NeuroTechnology MegaMatcher v 5.0 was used to analyze facial images from WVU's 2012 Biometric collection project comprising of 1200 participants of both genders from various ethnicities and age groups. However, this thesis exclusively concentrates on neutral pose facial images of participants captured under well controlled environment. MatchScores were generated using 'VeriLook' component of this commercial matcher then genuine and impostor match score distributions were developed using these scores.

Genuine score distributions were generated by matching a single probe image from an individual against a gallery of all images of the same individual and this process was repeated until every gallery image acts as a probe. Similarly, imposter score distributions were generated by matching a single probe image against all other images from participants in the dataset. But, this matcher experienced 'out of memory' exception due to large file size of facial images. To overcome this, a random subset of gallery was used to generate impostor match scores. For both genuine and imposter scores, gallery excludes the probe images.

Considering overall data and after demographic breakdown, the match score distributions exhibited an overlap between genuine and impostor distributions. From the observed false rejection at false acceptance rates of $1 \%$, FRR was observed lesser within demographic cohorts than in entire dataset except in age group 50-70. However, receiver operating characteristic (ROC) curves were plotted to better understand this dataset. For all the three demographics: gender, age and ethnicity, greater area under curve (AUC) and lower equal error rate (EER) were observed with respect to overall data which is desirable. Further examining the ROC curves, male cohort performed better than female and age group 20-30 exhibited better performance among age demographics while age 50-70 being worse. Among ethnicities, almost ideal performance was 
shown by Africans, Asian, Caucasians, Middle-Eastern and Pacific-Islanders while 100\% accuracy was seen in other ethnic groups.

Kullback-Leibler divergence and Jensen-Shannon divergence were implemented to measure the distance scores of each demographic from overall data. The distances between impostor distributions of overall data and each demographic was seen lesser in age demographics compared to gender and ethnicities. Among impostors, distance measures of Africans is higher, while it is much lesser in Caucasians, 'Others' and all age groups. In genuine case, distance measure of male cohort, age group 20-30 and Caucasians from overall data is much lesser than those in remaining demographics while it is significantly higher for 'Pacific-Islanders' because of its smaller sample size. Using KLD and JSD, almost analogous trend was observed in both genuine and impostor match cases for all demographics.

After observing the match score distributions of the facial images in this dataset, we can say that this matcher reflected baseline performance of a biometric system. Demographic breakdown can be said crucial while analyzing this dataset after observing the ROC curves. Although, this may not be necessarily true for every dataset, demographic breakdown to some extent may help in evaluating larger biometric datasets of this kind. However, prior to the release of such datasets as reference databases for real-time applications, it is important to cross check obtained results before drawing conclusions rather than concluding just by evaluating results from a single matcher. Also, it was observed that matcher yielded high failure to enroll rates in certain demographics. This may not be the case on implementing another matcher.

\subsection{Future Work}

This work can be extended in multiple ways. Firstly, in order to end up with robust results, evaluating and cross checking the data with other matcher is always recommended. Secondly, multi-biometrics can be performed with this dataset since it contains iris and fingerprint images similar to facial images. Multi-biometrics adds strength while evaluating a dataset. Thirdly, it is always challenging to work with non-neutral pose images over neutral poses which may yield robust matching results. Also, working on occluded images and images captured under uncontrolled environments is something to be done eventually. Finally, as 3D is prevailing over $2 \mathrm{D}$ in current state-of-art, concentrating on 3D adds strength to this field 


\section{Appendix}

\section{Ethnicity Match Score Distributions for Face Data}

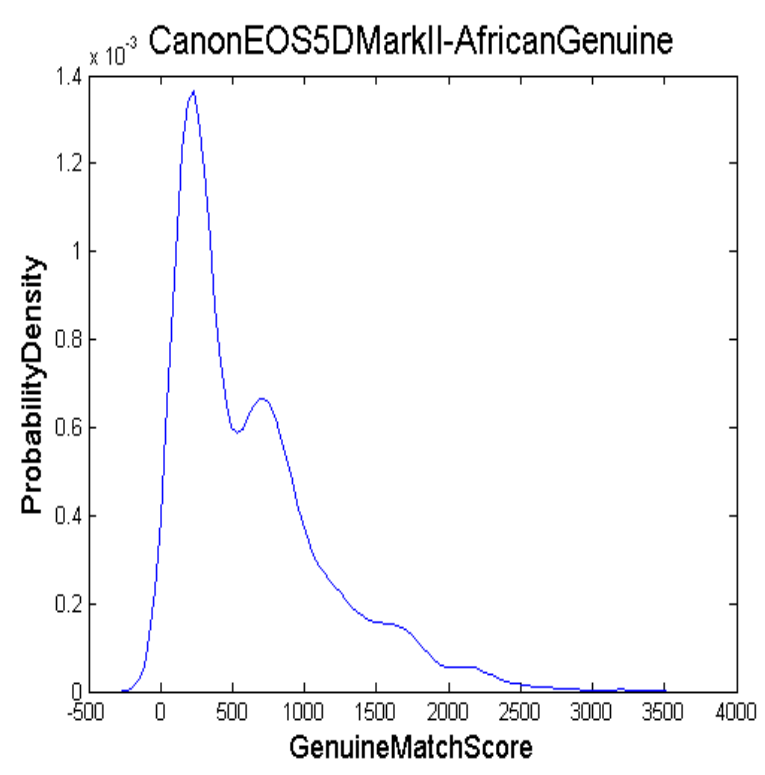

(a)

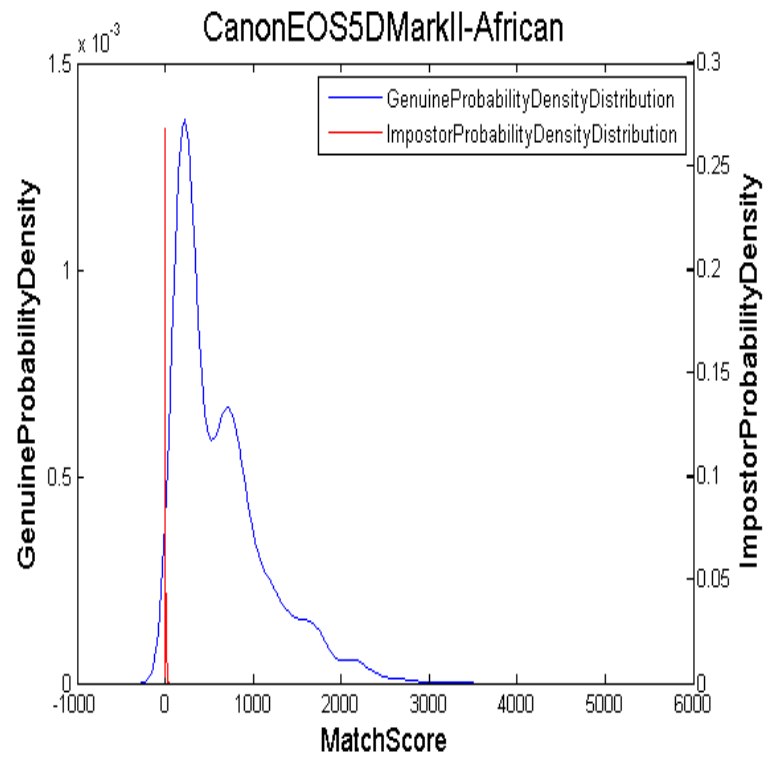

(c)

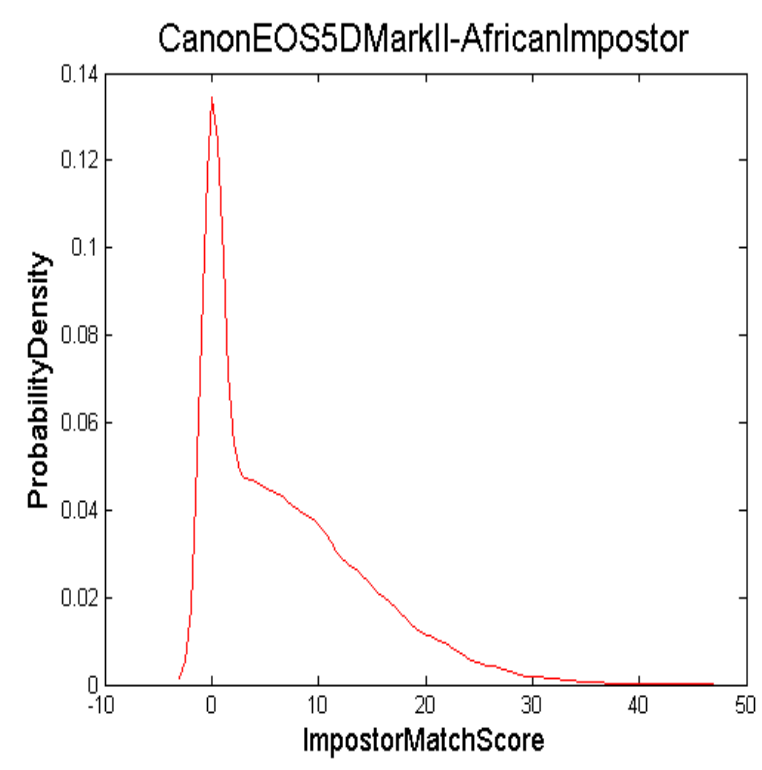

(b)

Figure A-1: Genuine and imposter score distributions for African face images from Canon EOS 5D Mark II sensor. (a) Genuine distribution of African face. (b) Impostor distribution of African face. (c) Combined distribution of match scores of African face. 


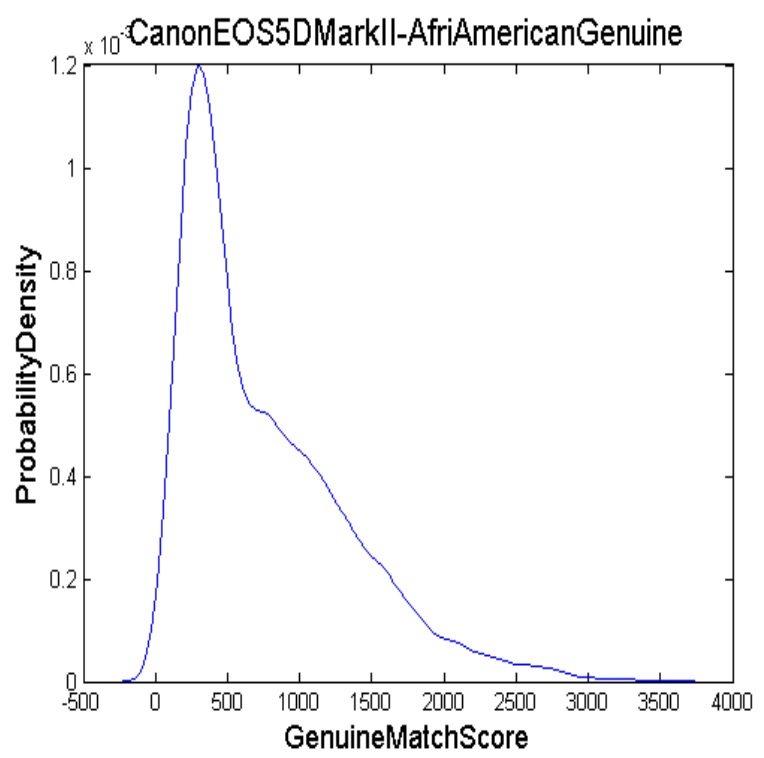

(a)

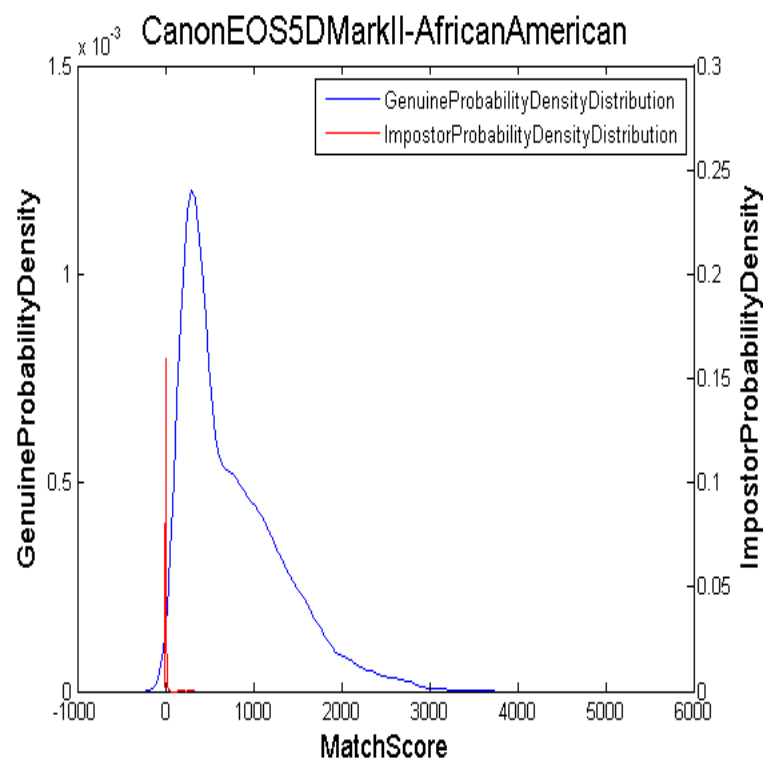

(c)

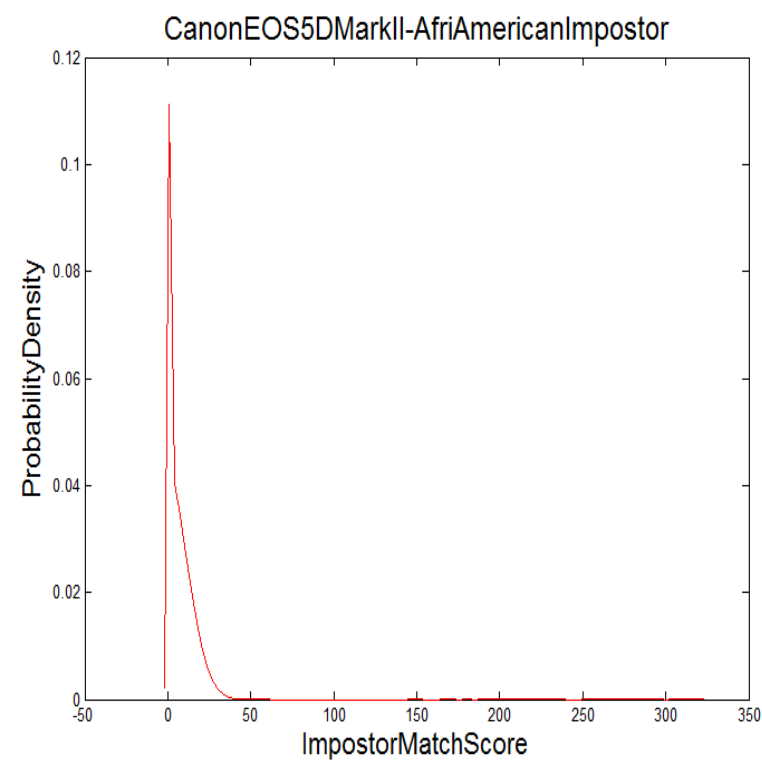

(b)

Figure A-2: Genuine and imposter score distributions for African-American face images from Canon EOS 5D Mark II sensor.

(a) Genuine distribution of African-American face. (b) Impostor distribution of African-American face. (c) Combined distribution of match scores of AfricanAmerican face. 


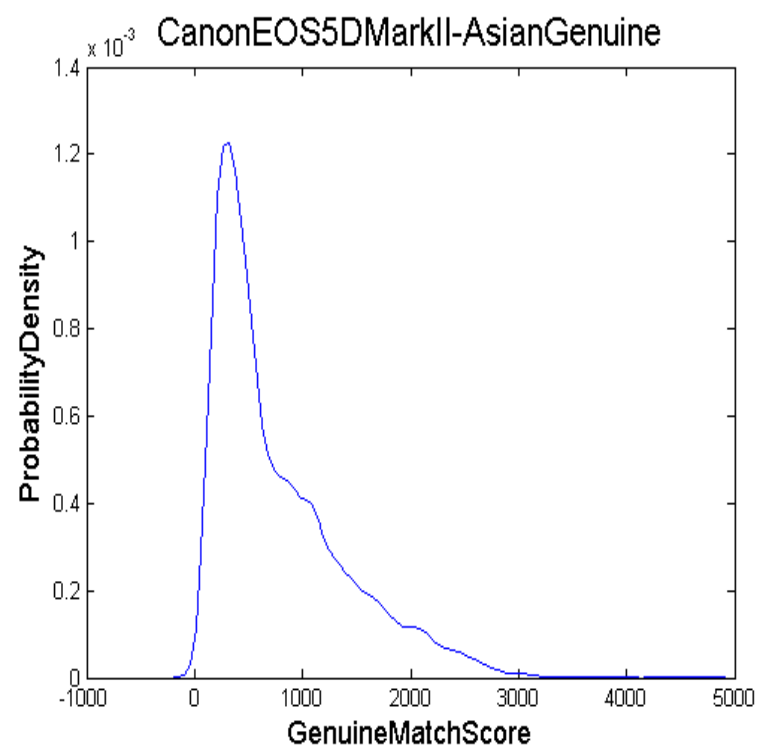

(a)

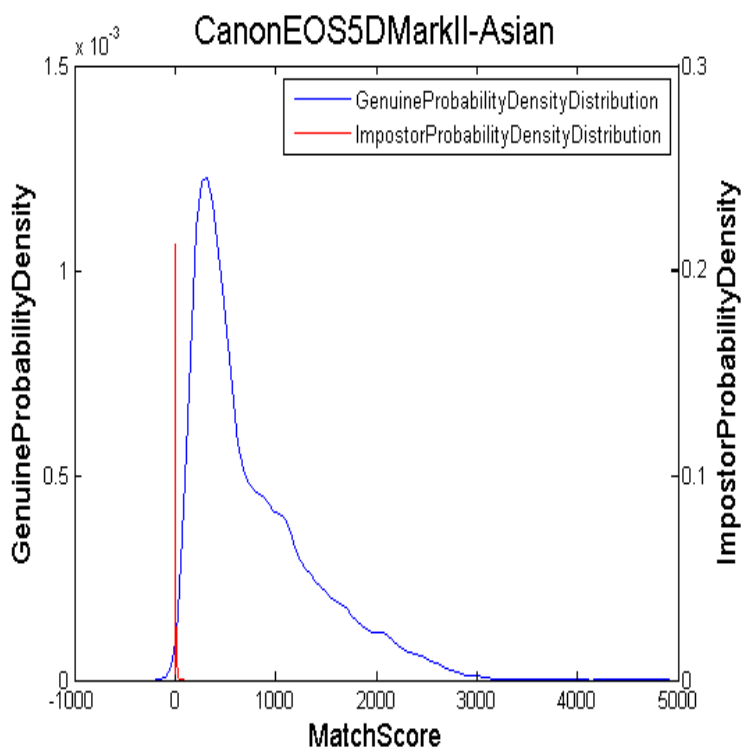

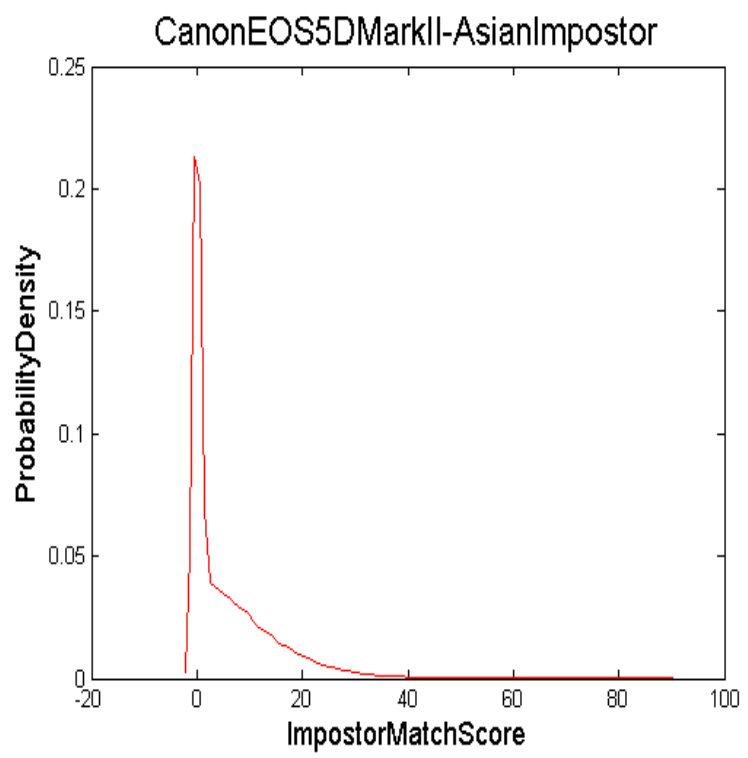

(b)

Figure A-3: Genuine and imposter score distributions for Asian face images from Canon EOS 5D Mark II sensor.

(a) Genuine distribution of Asian face. (b) Impostor distribution of Asian face. (c) Combined distribution of match scores of Asian face.

(c) 


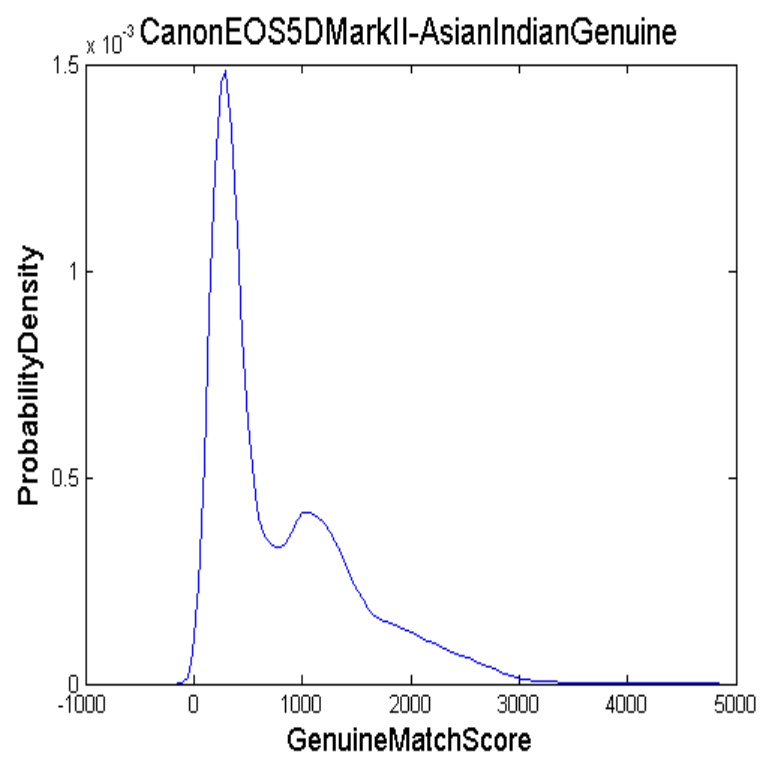

(a)

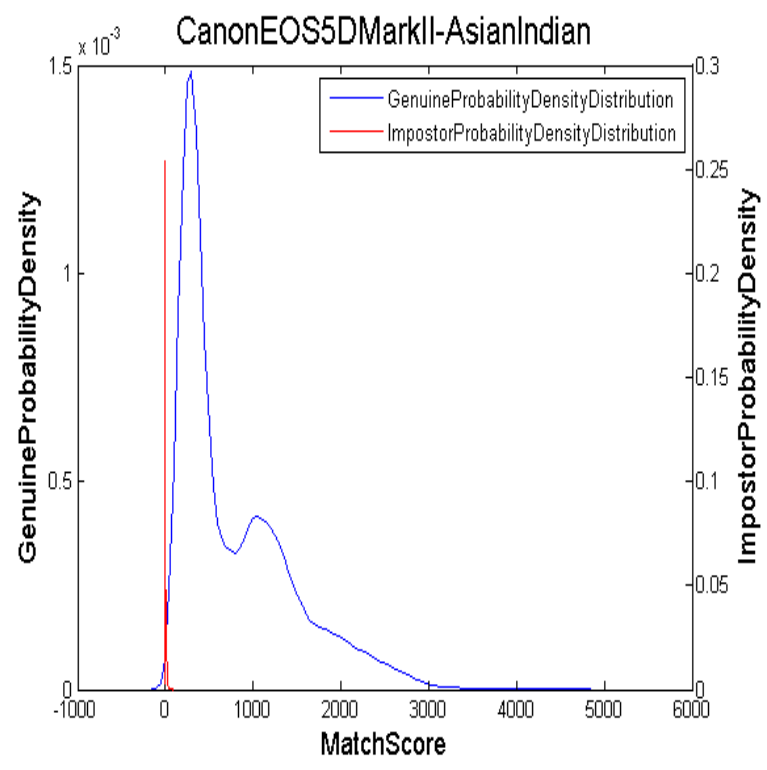

(c)

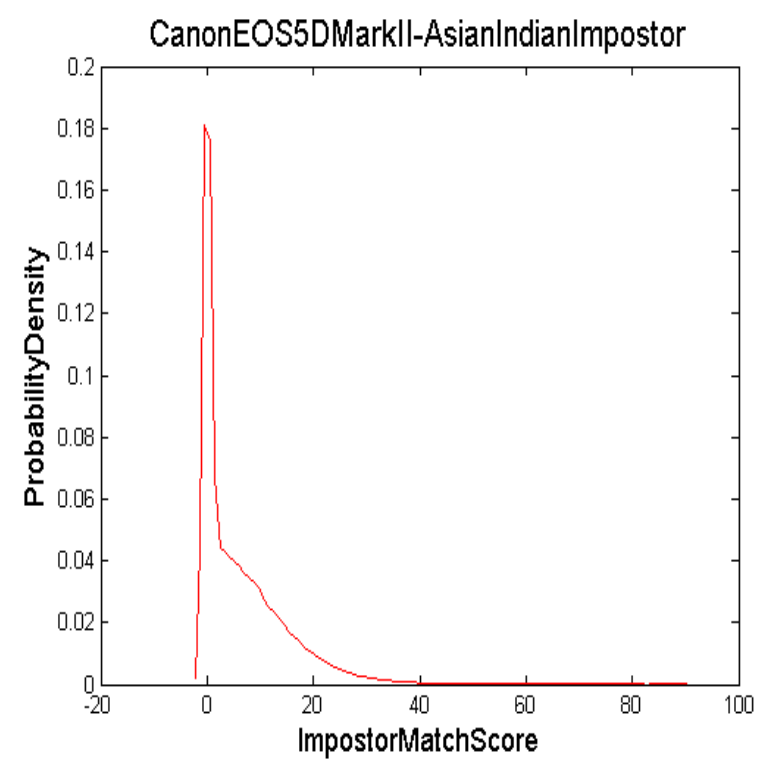

(b)

Figure A-4: Genuine and imposter score distributions for Asian-Indian face images from Canon EOS 5D Mark II sensor. (a) Genuine distribution of Asian-Indian face. (b) Impostor distribution of Asian-Indian face. (c) Combined distribution of match scores of Asian-Indian face. 


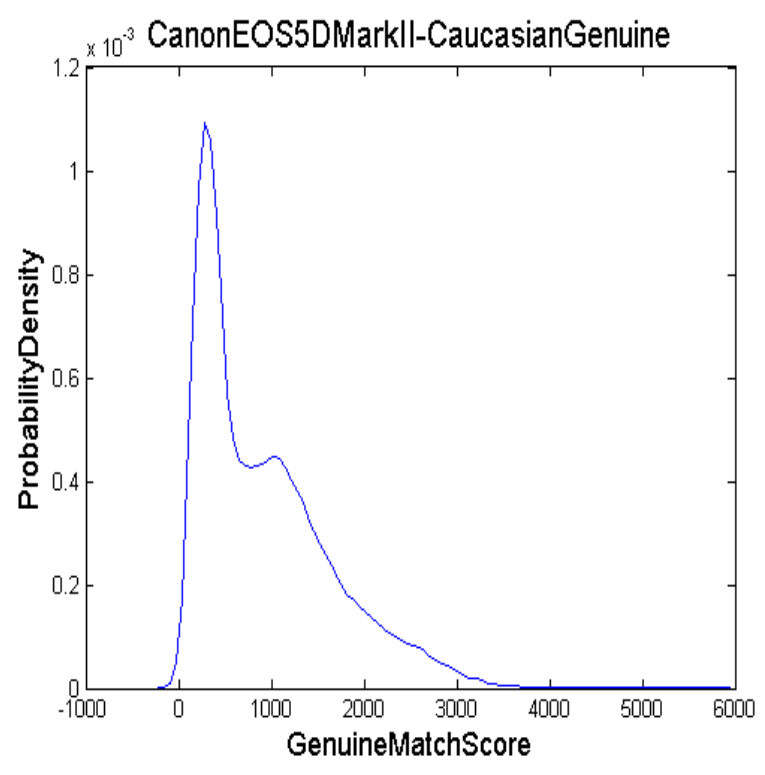

(a)

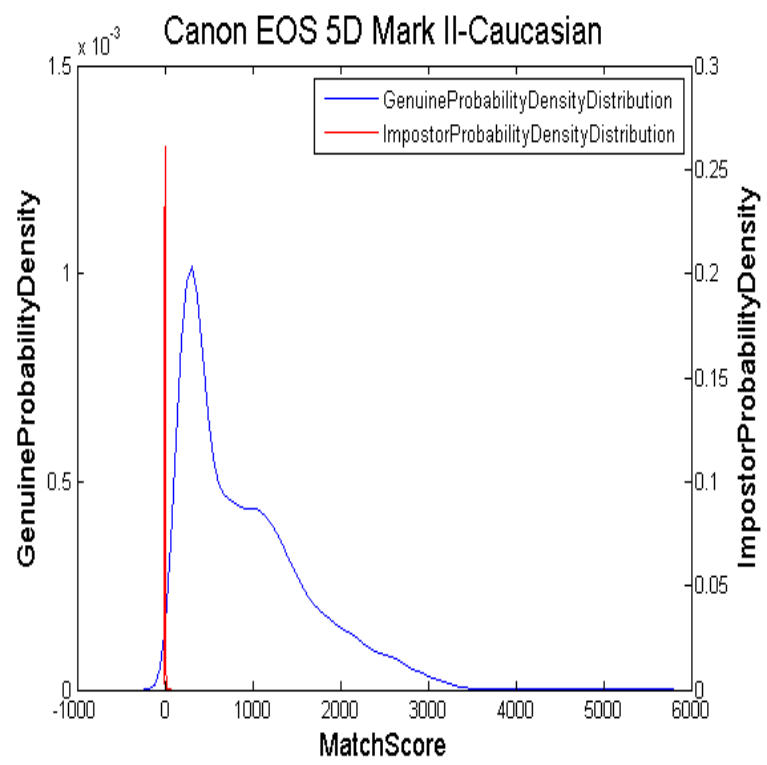

(c)

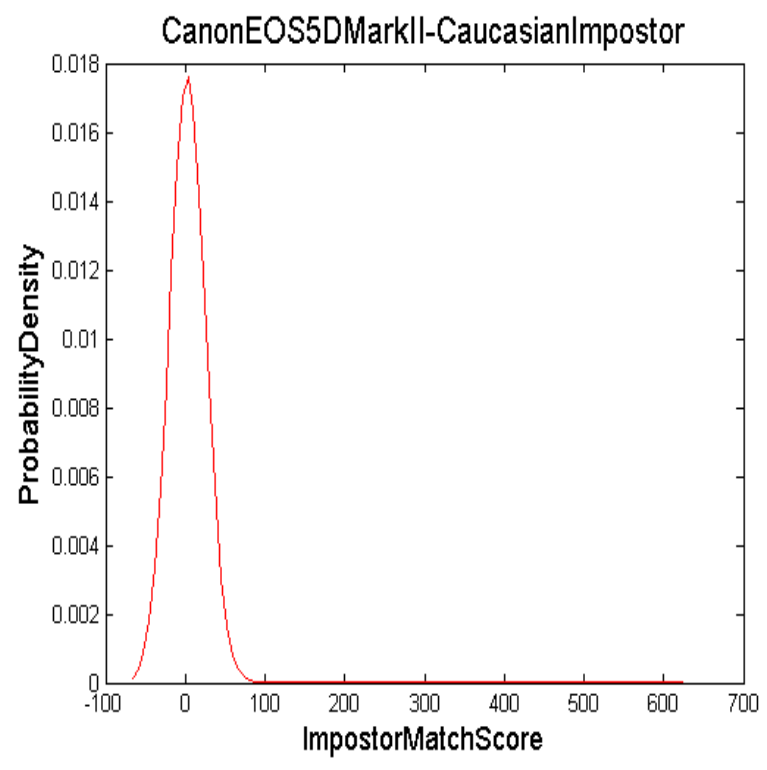

(b)

Figure A-5: Genuine and imposter score distributions for Caucasian face images from Canon EOS 5D Mark II sensor. (a) Genuine distribution of Caucasian face. (b) Impostor distribution of Caucasian face. (c) Combined distribution of match scores of Caucasian face. 


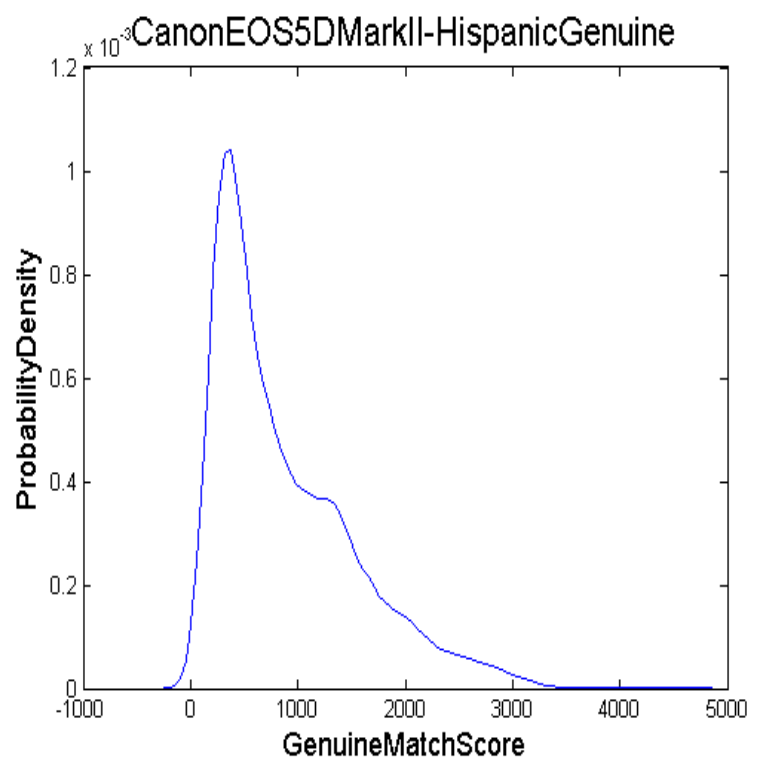

(a)

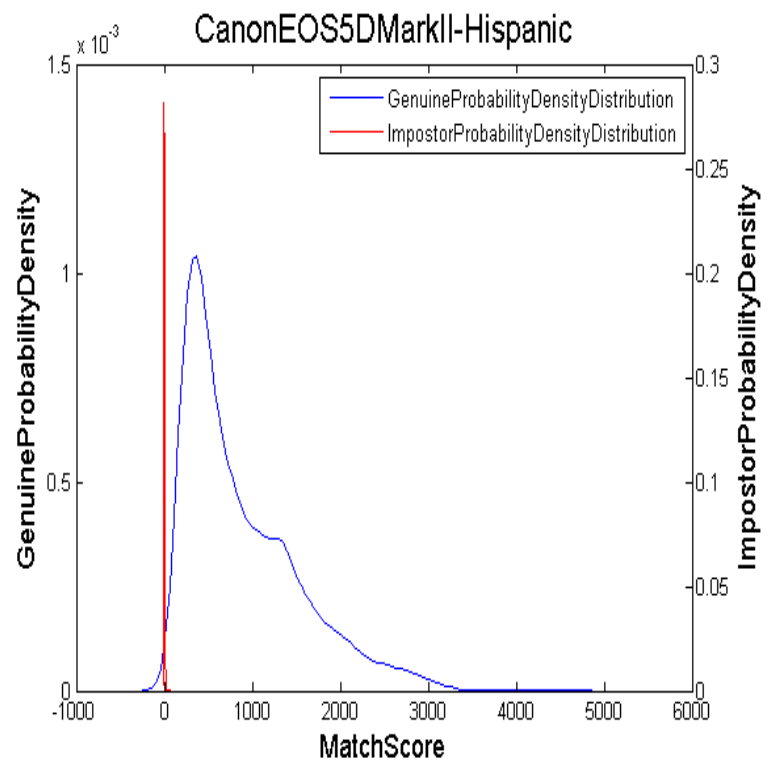

(c)

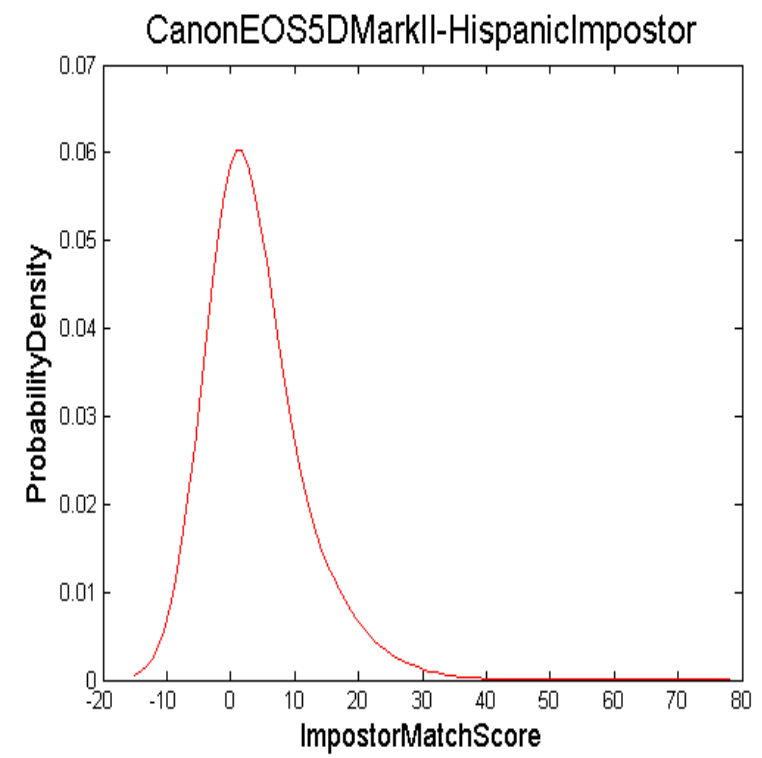

(b)

Figure A-6: Genuine and imposter score distributions for Hispanic face images from Canon EOS 5D Mark II sensor. (a) Genuine distribution of Hispanic face. (b) Impostor distribution of Hispanic face. (c) Combined distribution of match scores of Hispanic face. 


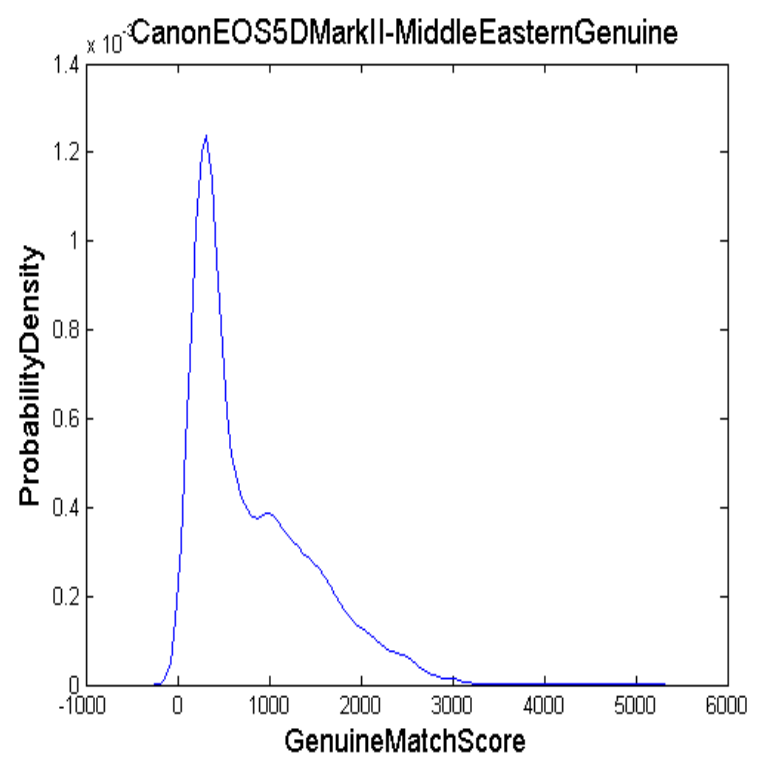

(a)

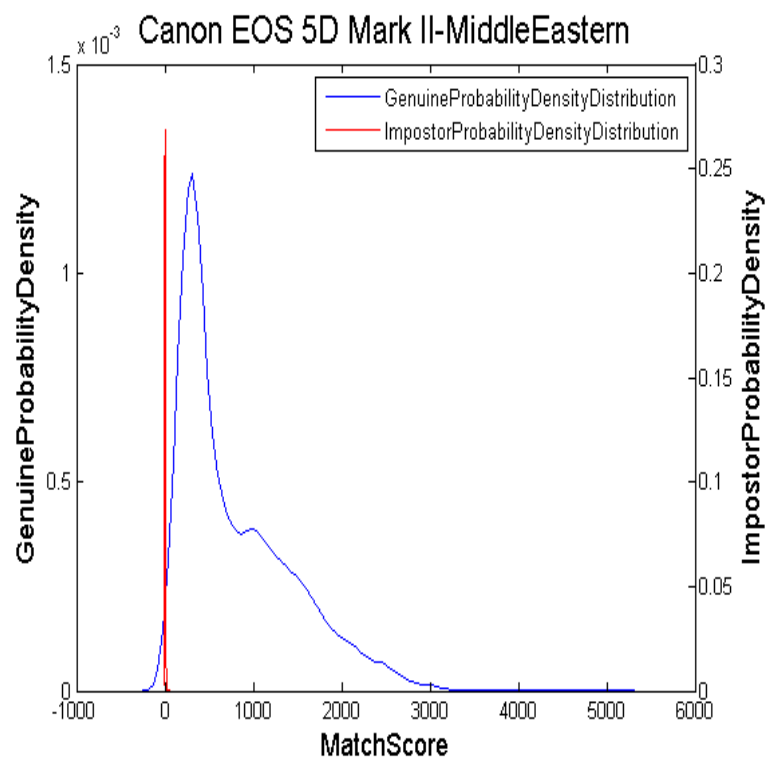

(c)

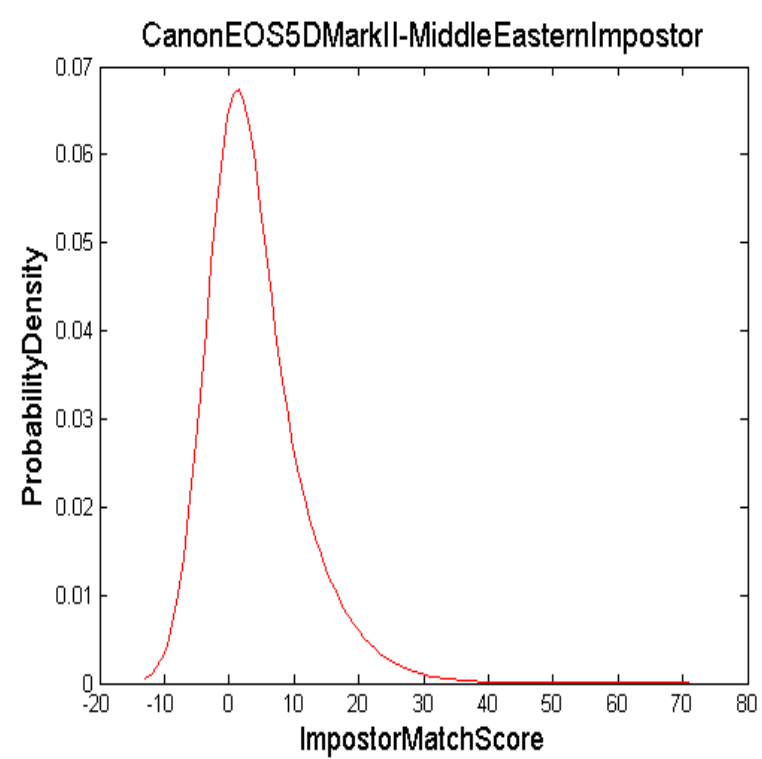

(b)

Figure A-7: Genuine and imposter score distributions for Middle-Eastern face images from Canon EOS 5D Mark II sensor.

(a) Genuine distribution of Middle-Eastern face. (b) Impostor distribution of Middle-Eastern face. (c) Combined distribution of match scores of MiddleEastern face. 


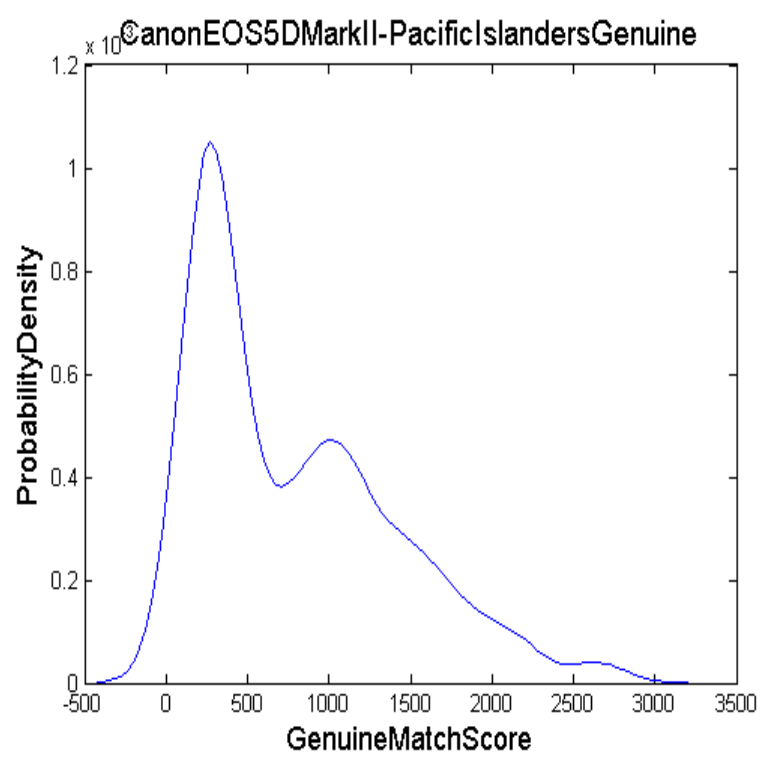

(a)

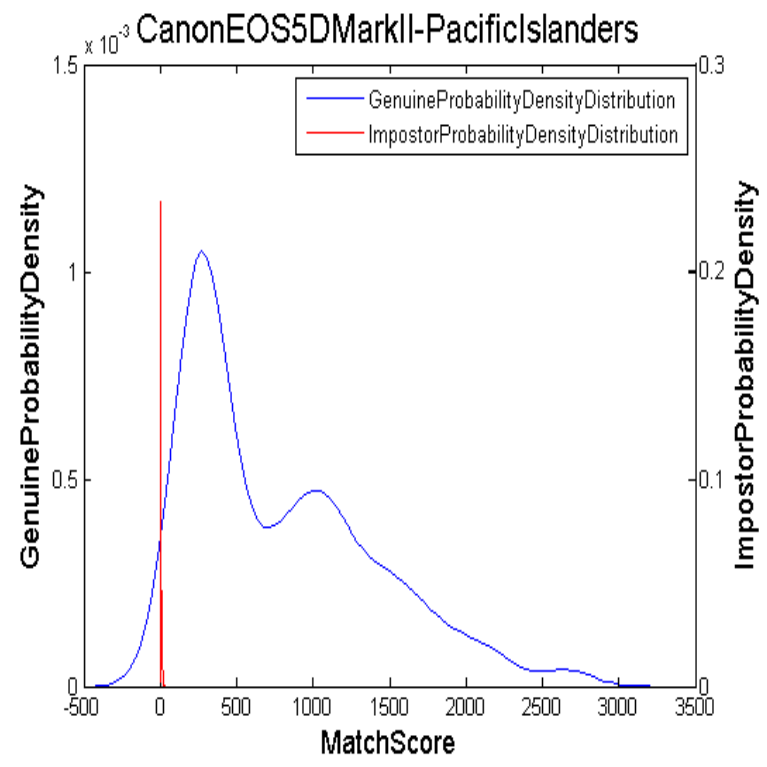

(c)

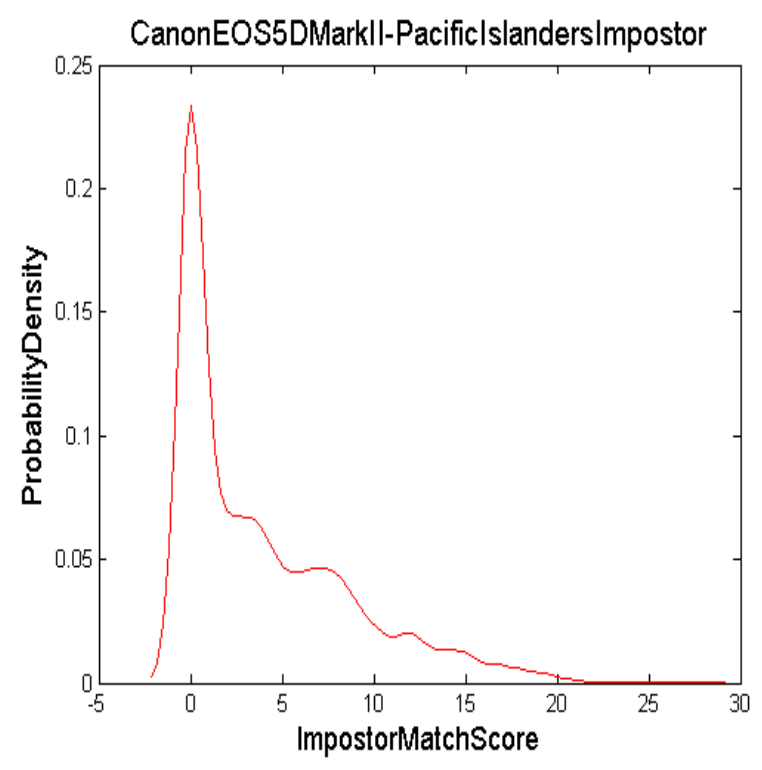

(b)

Figure A-8: Genuine and imposter score distributions for Pacific Islanders face images from Canon EOS 5D Mark II sensor.

(a) Genuine distribution of Pacific Islanders face. (b) Impostor distribution of Pacific Islanders face. (c) Combined distribution of match scores of Pacific Islanders face. 


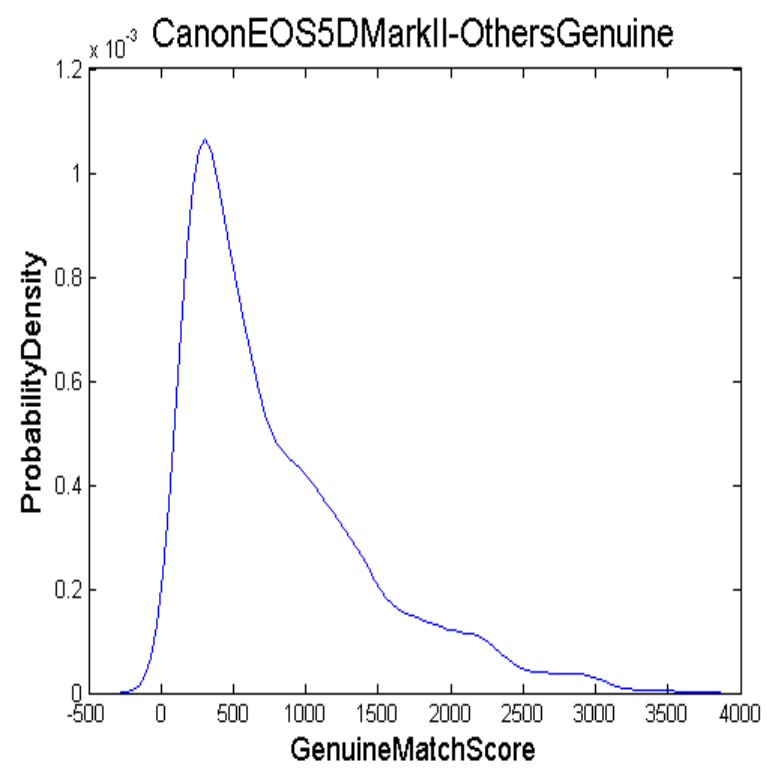

(a)

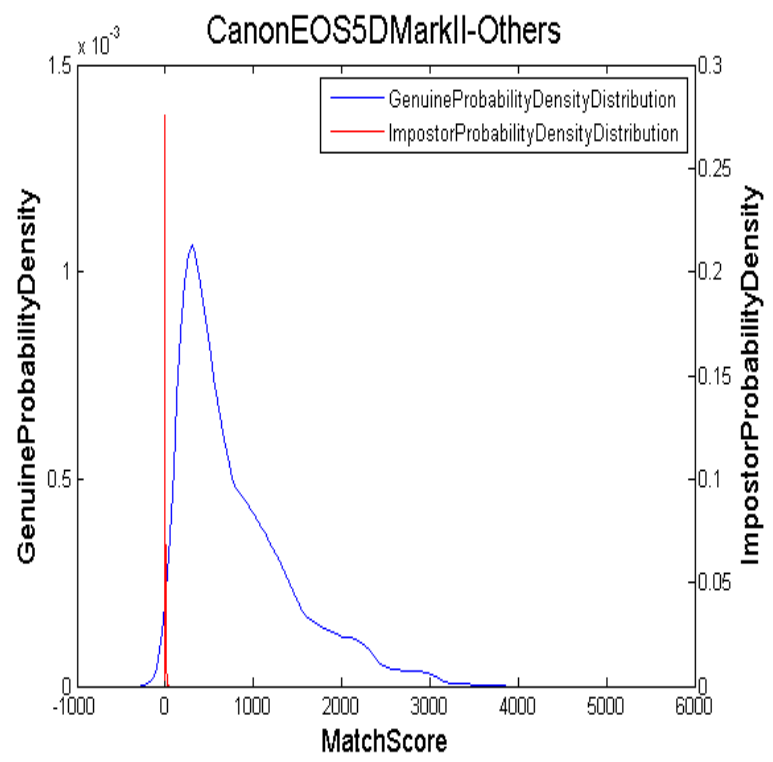

(c)

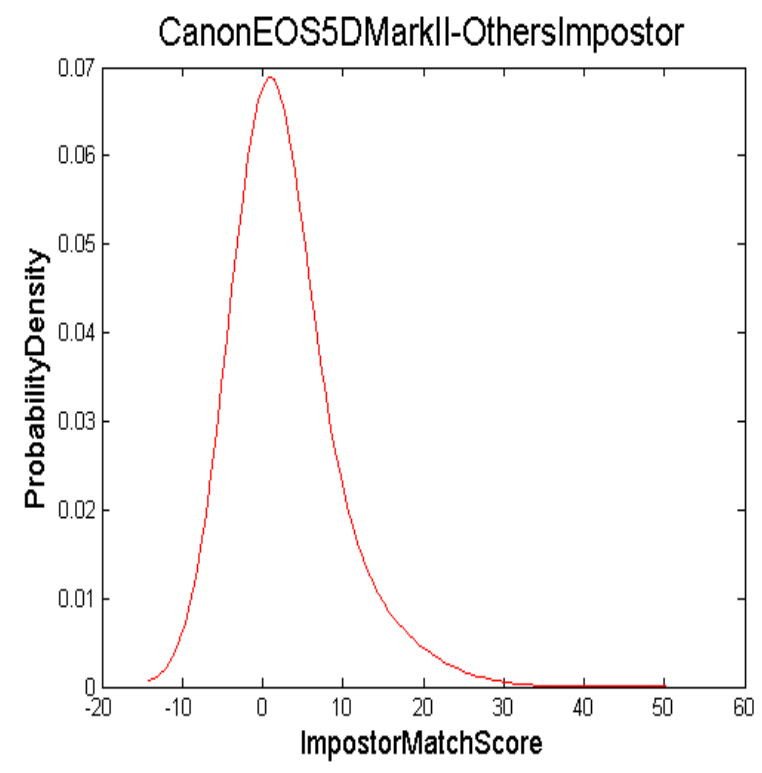

(b)

Figure A-9: Genuine and imposter score distributions for 'Others' face images from Canon EOS 5D Mark II sensor.

(a) Genuine distribution of 'Others' face. (b) Impostor distribution of 'Others' face. (c) Combined distribution of match scores of 'Others' face. 


\section{References}

[1] R.Hietmeyer, "Biometric identification promises fast and secure processing of airline passengers," The Int'l Civil Aviation Organization Journal, vol. 55, no. 9, pp. 10-11, 2000.

[2] A.Lanitis, C. J. Taylor and T.F. Cootes,, "Towards simulation of aging effects of face images," IEEE Trans. Pattern Analysis and Machine Intelligence, vol. 24, no. 4, 2002.

[3] W.Zhao, R. Chellappa, "Face Recognition: A literature survey.," ACM Computing Surveys, pp. 399-458, 2003.

[4] Ambika Ramchandran, Ravindra Kumar, "Overview of face recognition system challenges," INTERNATIONAL JOURNAL OF SCIENCE\&TECHNOLOGY RESEACH, vol. 2, no. 8, 2013.

[5] M. Kirby and L.Sirovich, "Application of the Karhunen-Loe`ve procedure for the charecterisation of human faces," IEEE Trans. Pattern Analysis and Machine Intelligence, vol. 12, pp. 831-835, 1990.

[6] M. Turk and A.Pentland, "Eigenfaces for recognition," J.Cognitive Neuroscience, vol. 3, pp. 71-86, 1991.

[7] Anil. K. Jain, Patric Flynn and Arun. A. Ross, Handbook of Biometrics, vol. 32, Springer, 2007, pp. 547-564.

[8] A.Pentland, B. Moghaddam, and T. Starner,, "View-Based and modular eigenspaces for face recognition," Proc. IEEE CS Conf. Computer Vision and Pattern Recognition, pp. 8491, 1994.

[9] P. Belhumeur, J. Hespanha and D. Kriegman, "Eigenfaces vs. Fisherfaces Recognition Using Class Specific Linear Projection," IEEE Transactions on Pattern Analysis and Machine Intelligence, PAMI, vol. 19, no. 7, pp. 711-720, 1997.

[10] A. M.Martinez, A. C. Kak, "PCA versus LDA," IEEE Trans. on Pattern Analysis and Machine Intelligence, vol. 23, no. 2, pp. 228-233, 2001.

[11] Stanz. Li and Anil. K. Jain, Handbook of Face Biometrics, vol. 23, Springer, 2005, pp. 228-233.

[12] S. Lawrence, C. L.Giles, A. C. Tsoi and A. D. Back, "Face Recognition A Convolutional Neural Network Approach," IEEE Trans. Neural Networks, vol. 8, pp. 98-113, 1997.

[13] S. H. Li, S. Y. Kung and L. J. Lin, "Face recognition/detection by probabilistic decisionbased neural network," IEEE Trans. Neural Networks, vol. 8, pp. 114-132, 1997. 
[14] Laurenz. Wiskott, Jean-Marc Fellous, Norbert Kruger and Christoph von der Malsburg, "FaceRecognition by Elastic Bunch Graph Matching," Proc. 7th Intern. Conf. on Computer Analysis of Images and Patterns, pp. 456-463, 1997.

[15] R. Bruneli and T. Poggio, "Face recognition: features versus templates," IEEE Trans. Pattern Analysis and Machine Intelligence, vol. 15, pp. 1042-1052, 1993.

[16] Ralph Gross, Iain Matthews, Jeffery Cohn, Takeo Kanade, Simon Baker, "Multi-PIE," Image and Vision Computing, vol. 28, pp. 807-813, 2010.

[17] Anil. K. Jain, Ajay Kumar, Biometrics of Next Generation: An Overview, Springer, 2011, pp. 373-378.

[18] Y. Gao and K. H. Leung, "Face Recognition using lineedge map," IEEE Transaction on Pattern Analysis and Machine Intelligence, vol. 24, no. 6, June 2002.

[19] A. S. Tolba, A. H. El-Baz and A. A. El-Harby, "Face Recognition-A Literature Review," World Academy of Science, Engineering and Technology, vol. 2, 2008-07-21.

[20] "Bern Univ. Face Database," Bern University, 2002. [Online]. Available: ftp://iamftp.unibe.ch/pub/Images/FaceImages/.

[21] "Purdue Univ. Face Database," Purdue University, 2002. [Online]. Available: http://rvl1.ecn.purdue.edu/ aleix/aleix_face_DB.html.

[22] G. Guo, S. Z. Li, K. Chan, "Face Recognition by Support Vector Machines," Proc. of the IEEE International Conference on Automatic Face and Gesture Recognition, pp. 196-201, 2000.

[23] K. Johnsson, J. Matas, J. Kittler and Y. P. Li, "Learning Support Vectors for Face Verification and Recognition," in Proc. of the IEEE International Conference on Automated Face and Gesture Recognition, Grenoble, France, pp. 208-213, 2000.

[24] Anil. K. Jain, Arun. A. Ross. Kartik Nandakumar, Introduction to Biometrics, Springer, 2011.

[25] "The AT\&T Database of Faces," [Online]. Available: http://www.uk.research.att.com/facedatabase.html.

[26] "The XM2VTS database," [Online]. Available: http://www.ee.surrey.ac.uk/Research/VSSP/xm2vtsdb/.

[27] "The Yale database," [Online]. Available: http://cvc.yale.edu/.

[28] "The Yale B database," [Online]. Available:

http://cvc.yale.edu/projects/yalefacesB/yalefacesB.html. 
[29] "The MIT face database," [Online]. Available: ftp://whitehapel.media.mit.edu/pub/images/.

[30] "The CMU PIE database," [Online]. Available: http://www.ri.cmu.edu/projects/project 418.html.

[31] "The FERET database," [Online]. Available: http://www.it1.nist.gov/iad/humanid/feret/.

[32] "Kuwait University Face Database," [Online]. Available: http://www.sc.kuniv.edu.kw/lessons/9503587/dina.html.

[33] P. J. Phillips, P. Grother, R. J. Michaels, D. M. Blackburn, E. Tabassi and. M. Bone, "Face recognition Vendor Test 2002: Evaluation Report," NISTIR 6965, NAT. Inst. of Standards and Technology, 2003.

[34] P. Jonathon Phillips, W. Todd Scruggs,Alice J.O' Toole, Patrick J. Flynn, Kevin W.Bowyer, Cathy L.Schott, Matthew Sharpe, "FRVT 2006 and ICE 2006 Large-Scale Results," NISTIR 7408, National Institute of Standards and Technology, March 2007.

[35] Introna ,L and Nissenbaum. H, "Facial Recognition Technology A Survey of Policy and Implementation Issues," The Department of Organisation, Work and Technology, Lancaster University, 2010.

[36] R. Michael McCabe and Elaine M. Newton, "American National Standards for Information Sysytems- Data Format for the Interchange of Fingerprint, Facial \& Other Biometric Information- Part 1," NIST Special Publication 500-271, USA, May, 2007.

[37] O. Deniz, M. Castrillon, M. Hernandez, "Face recognition using independent component analysis and support vector machines," Pattern Recognition Letters, vol. 24, pp. 21532157, 2003.

[38] Michael J. Johns, Paul A. Viola, "Robust Real-Time Face Detection," International Journal of Computer Vision, no. 57(2), pp. 137-154, 2004.

[39] G. Guo, "Face Detection and Recognition," 2014. [Online]. Available: http://www.csee.wvu.edu/ gdguo/courses/fa14/biom426/Slides/Lecture9.pdf.

[40] National Science and Technology Council, "Biometrics 'Foundation Documents'," [Online]. Available: http://www.biometrics.gov/documents/biofoundationdocs.pdf.

[41] J. Chang- yeon, "Face Detection using LBP features," December 2008. [Online]. Available: http://cs229.stanford.edu/proj2008/Jo-FaceDetectionUsingLBPfeatures.pdf.

[42] Romuere Silva, Kelson Aires, Rodrigo Veras, Thiago Santos, Kalyf Lima and Andre` Soares "Automatic Motorcycle Detection on Public Roads," CLEI Electronic Journal, vol. 16, no. 3, 2013. 
[43] M. Osborne, "CFCS- Entropy and Kullback- Leibler Divergence," University of Edinburg, February 2008. [Online]. Available: http://www.inf.ed.ac.uk/teaching/courses/cfcs1/lectures/entropy.pdf.

[44] J. Hooks, "Validating the Representativeness of Test Samples for Performance Prediction of Face Recognition," ProQuest, Masters Thesis, December 2015.

[45] S. Zhou, "Bayesian Model Selection in terms of Kullback-Leibler discrepancy," PhD Thesis, Columbia University, 2011.

[46] N. Schmid, "“Performance Evaluation-BIOM-426"," 2009. [Online]. Available: http://www.csee.wvu.edu/ natalias/biom426/performance_fall09.pdf.

[47] "Using the Receiver Operating Charcteristics (ROC) to analyze a classification model," [Online]. Available: http://www.math.utah.edu/ gamez/files/ROC-Curves.pdf.

[48] X. Lu, Image analysis for face recognition, May 2003.

[49] B. Moghaddam, "Principal manifolds and probabilistic subspaces for visual recognition," IEEE Transactions on Pattern Analysis and Machine Intelligence, vol. 24(6), pp. 780-788, June 2002.

[50] D. Cai, X. He, J. Han, "Semi-supervised discriminant analysis. Computer Vision," IEEE 11th International Conference on Computer Vision, vol. 14, pp. 1-7, 2007.

[51] C. Liu and H. Wechsler "Comparative assessment of independent component analysis (ica) for face recognition," in In Proc. of the Second International Conferenceon Audio- and Video-based Biometric Person Authentication, AVBPA'99, Washington D.C., USA, March 1999.

[52] Ali-M.Al-Khouri, "Critical Insights-From a Practitioner Mindset," vol. 3, p. 88, 2012.

[53] "NeuroTechnology. MegaMatcher 5.0, VeriFinger7.0, VeriLook 5.5, VeriEye 2.8 and VeriSpeak 2.1 SDK," NeuroTechnology, November 2014. [Online]. Available: http://www.neurotechnology.com/download.html.

[54] Amanda J. Sgroi, "Exploration of Impostor Score Distribution for Face-Based Biometrics," PhD Thesis, University of Notre Dame, Indiana, April 2015.

[55] Juan Fransisco G`omez-Lopera, Jos`e Mart`inez-Aroza, Aureliano M. Robles-P`erez and Ram`on Rom`an-Rold`an, "An Analysis of Edge Detection by Using Jensen-Shannon Divergence," Journalof Mathematical Imaging and Vision, vol. 13, pp. 35-56, 2000.

[56] Gavin E Crooks, David A Sivak, "Measure of trajectory ensemble disparity in nonequilubrium statistical dynamics," Journal of Statistical Mechanics: Theory and Experiment, 2011. 
[57] T. Yamano, "A Note on Bounds for Jensen-Shannon Divergence by Jefferys," in 1st International Electronic Conference on Entropy and it's Applications, Japan, November, 2014.

[58] Charles Otto, Hu Han, and Anil Jain, "How Does Aging Affect Facial Components?," Michigan State University, Michigan, USA.

[59] Brendan F. Klare, Mark J. Burge, Joshua C. Klontz, Richard W. Vorder Bruegge, Anil K. Jain, "Face Recognition Performance: Role of Demographic Information," IEEE Transactions on Information Forensics and Security.

[60] Dmitry O. Gorodnichy, Eric Granger, Paolo Radtke, "Survey of Commercial Technologies for Face Recognition in Video," DRDC Centre for Security Science, Ottawa, Canada, September, 2014.

[61] P. J. Phillips, "Support vector machines applied to face recognition," In Processing system 11,, 1999. 